Aus der Klinik für Dermatologie, Venerologie und Allergologie

im Zentrum Arbeits-, Sozial-, Umweltmedizin und Allergologie

(Prof. Dr. med. M. P. Schön)

der Medizinischen Fakultät der Universität Göttingen

\title{
Hautveränderungen im Gesicht: kognitive Wahrnehmung und emotionale Reaktion - eine Analyse des Betrachtungsverhaltens unter Erprobung der Restricted Focus View (RFV)-Technik
}

\author{
I NAUG URAL - DISSERTATION \\ zur Erlangung des Doktorgrades \\ der Medizinischen Fakultät der \\ Georg-August-Universität zu Göttingen
}

vorgelegt von

Norman Voigt

aus

Neubrandenburg

Göttingen 2014 
Dekan: Prof. Dr. rer. nat. H. K. Kroemer

I. Berichterstatter: Prof. Dr. med. Thomas Fuchs

II. Berichterstatter: Prof. Dr. med. Jürgen Müller

III. Berichterstatter:

Tag der mündlichen Prüfung: 16.03.2015 


\section{Inhaltsverzeichnis}

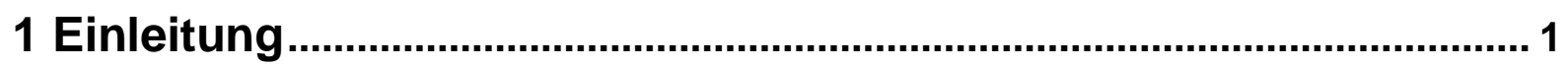

1.1 Forschungsperspektiven zum Problem Entstellung .................................... 1

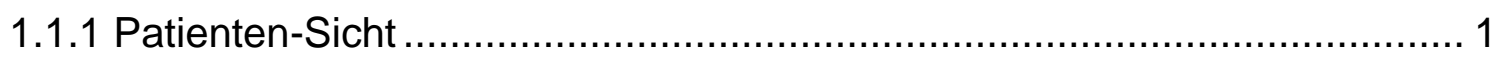

1.1.2 Psychosoziale Perspektive ............................................................. 2

1.2 Visueller Reiz - fragliche Bedeutung für Entstellungs-Urteile ........................ 2

1.3 Das Schema-Konzept als Ansatz in der Entstellungsforschung........................ 3

1.4 Erfassung von Blickverhalten ................................................................ 6

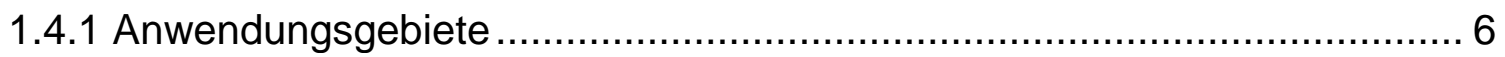

1.4.2 Unterschiedliche Verfahren zur Blickregistrierung ................................. 7

1.4.3 Die Attention-Tracking-Methode ……................................................... 7

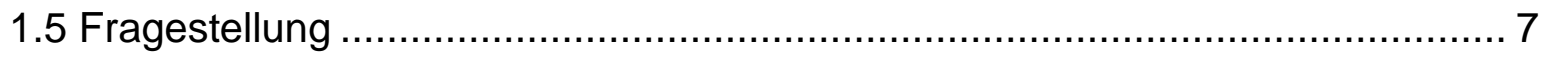

2 Material, Methoden und Durchführung ............................................... 9

2.1 Reizstimuli: Auswahl der Portraitfotos ........................................................ 9

2.2 Stichprobe der Versuchspersonen ....................................................... 11

2.3 Untersuchungsmethode: der Restricted Focus Viewer (RFV) ...................... 12

2.3.1 Funktion und Anwendungsgebiet ................................................... 12

2.3.2 Zur allgemeinen Validität des RFV ................................................. 15

2.3.3 Filterung der Bildvorlagen - Trennung diagnostischer und navigatorischer Informationen .................................................................. 16

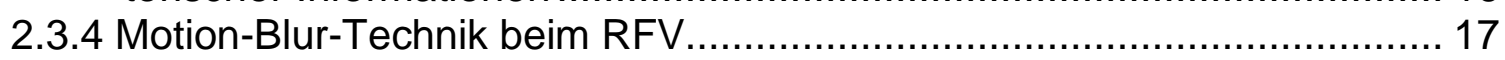

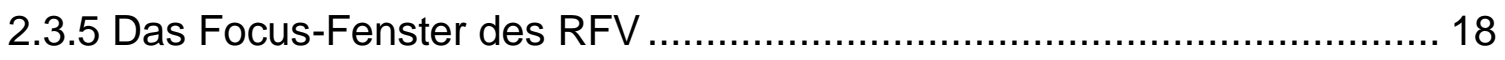

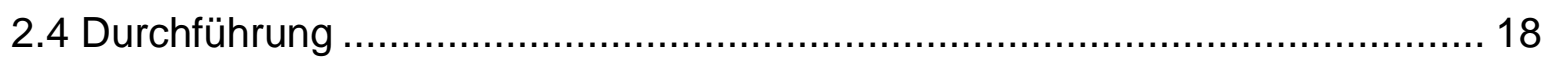

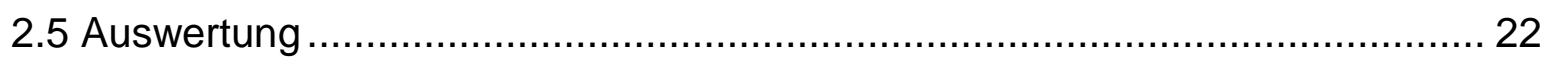

3 Darstellung der Ergebnisse............................................................... 25

3.1 Prüfung der unabhängigen Variablen und thematisch-relevante

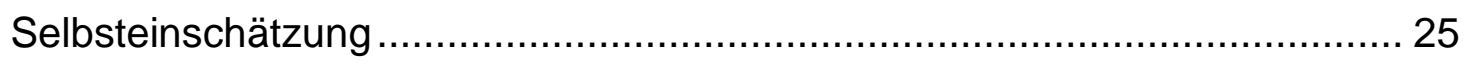

3.2. Eignung des RFV-Verfahrens (Hypothese 1) ......................................... 28

3.2.1 Verbale Einschätzungen............................................................... 28

3.2.2 Bildhafte Wiedergabe der Effektivität des RFV-Verfahrens ..................... 29

3.2.3 Erste numerische Wiedergabe und Auswertung von Blickzeiten............... 33

3.3 Blickverhalten und das Schema-Konzept (Hypothese 2) .............................. 35 
3.3.1 Aufmerksamkeitsveränderungen im Blickverhalten aufgrund von Hautveränderungen

3.3.2 Aufmerksamkeitsveränderungen als Folge der WahrnehmungsVerarbeitung

3.4 Beziehung Stigma-Fixationszeit und Bewertung (Hypothesen 3a, 3b, 3c) ..... 42

3.4.1 Eingehende Überprüfung der Bewertungen: Präsentationsfolge-Effekt und Dimensions-Unabhängigkeit.

3.4.2 Hypothesen-Testung: Korrelationen zwischen Stigma-ArealFixationszeit und Bewertungen

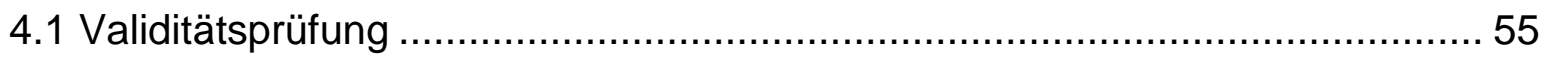

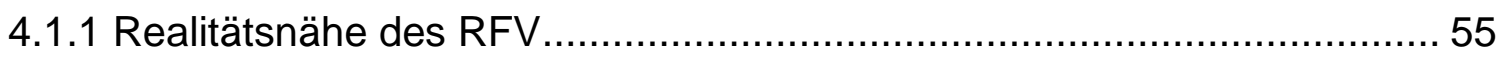

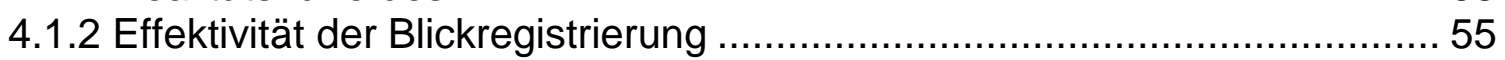

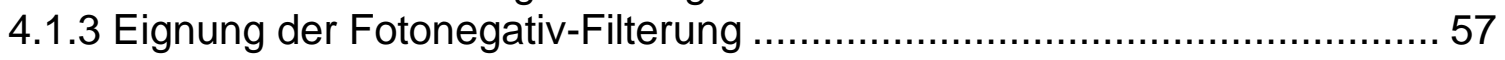

4.1.4 Auswirkungen der Expositionszeit ................................................... 58

4.1.5 Einfluss des Bildmaterials.............................................................. 58

4.1.6 Spezielle Zusammenstellung der Versuchsgruppe ............................... 59

4.2 Vergleich der RFV-Technik mit Eye-Tracking-Verfahren .............................. 59

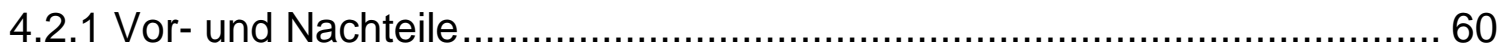

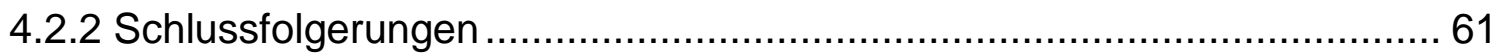

4.3 Überprüfungen der aufgestellten Hypothesen ............................................ 63

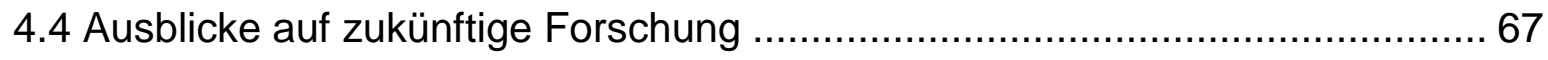

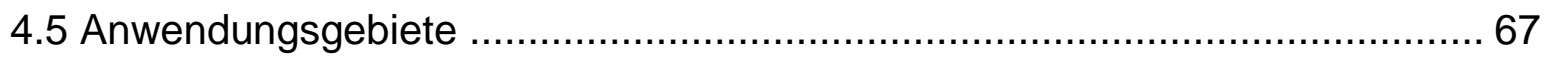

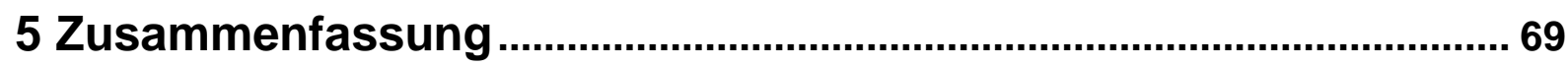

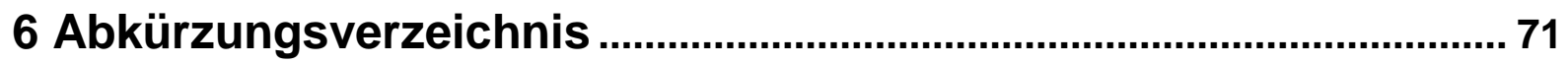

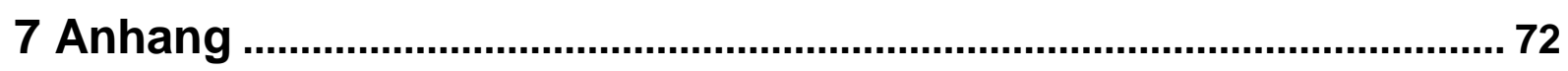

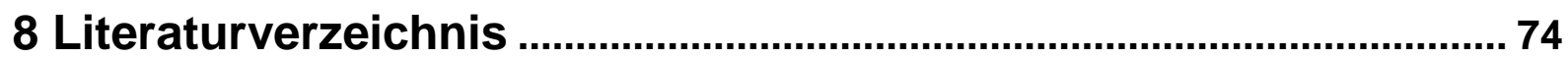




\section{Einleitung}

Mit vielen dermatologischen Veränderungen geht ein Gefühl von „Entstellung“ einher. Dies bezieht sich insbesondere auf Erkrankungen im Gesicht. Ein wesentlicher Faktor bei der Entstehung dieser Gefühle bei Betroffenen ist, neben individuell variablen persönlichen und charakterlichen Eigenschaften, die Wahrnehmung eines veränderten Blickverhaltens der Umwelt. In Kombination mit dem Wissen um eigene Abweichungen und sensibilisierenden kognitiven Prozessen resultiert nicht selten das Gefühl, „angestarrt“ zu werden.

Mit dieser Studie soll das Blickverhalten von Betrachtern, welches potentiell bei Betrachteten zu dieser Empfindung des „Anstarrens“ führt, analysiert, objektiviert und die daran geknüpfte „gefühlte Abwertung“ überprüft werden.

\subsection{Forschungsperspektiven zum Problem Entstellung}

Um die Problematik der „Entstellung“ zu erfassen, muss zwischen den verschiedenen, hierfür relevanten Ebenen der Interaktion unterschieden werden.

\subsubsection{Patienten-Sicht}

Patienten-zentrierte Forschung beschäftigt sich dabei zum einen mit der Selbstsicht der Betroffenen und zum anderen mit deren Interpretationen von Reaktionen anderer auf sie. Dabei stehen die Wertungen und Gefühle der jeweiligen Hautkranken im Fokus des Interesses. Eine Verlagerung des Interesses hin zu diesem Forschungsfeld stellten bereits Thompson und Gardner (2002) fest.

So kamen Studien auf diesem Gebiet zu dem Ergebnis, dass die Haut und ihre Beschaffenheit einen großen Anteil an der Bildung des Selbstwertgefühls und Selbstbewusstseins haben. Medizinisch-psychologisch relevant werden Hauterkrankungen dadurch, dass sie zu psychischen und psychosozialen Problemen führen und in einigen Fällen sogar die Grundlage depressiver Störungen sein können, unabhängig von ihrer Entität (Schubert 1989). Wichtiger als die Art ist die Lokalisation der Erkrankung. Exponierte, also sichtbare betroffene Areale wie zum Beispiel an Armen und Beinen oder vor allem im Gesicht vermitteln - besonders in 
Kombination mit einem entsprechend verändertem Blickverhalten der Interaktionspartner - das Gefühl „angestartt" zu werden und „objektiv entstellt" zu sein (Hünecke und Bosse 1980).

\subsubsection{Psychosoziale Perspektive}

Es ist jedoch schon hier wichtig, grundsätzliche Unterschiede zwischen der Eigenund Fremdsicht zu betonen, „denn von den Gedanken nimmt die Seele ihre Farbe an" wie schon Marc Aurel wusste (Selbstbetrachtungen, Buch 5, 16).

Ergebnisse zur Forschung am Sozialverhalten von dermatologisch sich "entstellt“ fühlenden Personen können dies in vielen Punkten bestätigen. Gefühle von Scham und Verlegenheit bestimmen danach nicht nur entscheidend das Erleben und Verhalten bei Interaktionen mit anderen (Schubert 1989), vielmehr können sie im Verlauf zu einer Sozialphobie bis hin zur Sozialparanoia führen (Fassheber et al. 1976), deren Kennzeichen unter anderem sozialer Rückzug und weitestgehende Vermeidung von Kontakten auf Grund erhöhter sozialer Furcht sind. So entsteht ein Teufelskreis, in dem die negativen Denkstrukturen fortwährend eine negative Deutung jeglicher Interaktionen und damit Bestätigung des ablehnenden Vorverdachts bewirken.

Für die Problematik des Entstellungsgefühls sind diese Erkenntnisse besonders relevant, da Veränderungen im Gesicht für jeden Interaktionspartner sichtbar sind und bei negativer Denkstruktur implizit als abwertend empfunden werden.

\subsection{Visueller Reiz - fragliche Bedeutung für Entstellungs- Urteile}

Eine ganz besondere fixe Verknüpfung wird im evolutionär-psychologischen Ansatz gesucht. Denn Schönheit mag im Auge des Betrachters liegen, jedoch hat sich das bewertende Bewusstsein dahinter in Jahrmillionen menschlicher Evolution herausgebildet. „Was als schön gilt, bestimmen die Adaptationen des Betrachters" (Buss 2004, S. 199).

Evolutionär oder zumindest historisch gesehen, scheint die Verknüpfung von Geist und Körper, wie sie schon Platon annahm, in unseren Denkstrukturen verankert zu 
sein. In ihr sehen u.a. Hoyningen-Süess (1995) und Allesch (2006) die eigene Negativsicht begründet, wonach mit einem „entstellten“ Äußeren ein "hässlicher" Geist einhergeht. Dies bedeutet nichts anderes, als dass es für die betroffenen Patienten selbst schwer vorstellbar scheint, bei eigenen Abweichungen von der äußeren Norm in den Augen anderer Personen als nicht hässlich empfunden zu werden (Panse 1970; Rumsey und Harcourt 2004).

Die dabei gleichzeitig unterstellte reizgesteuerte Wahrnehmung greift Hünecke (2009) in „Entstellung - Plädoyer für einen wahrnehmungspsychologischen Ansatz“ auf.

Auch Untersuchungen von Hassebrauck und Küpper (2002) konnten zeigen, dass Bedingungen auf der Stimulus-Seite, im hier vorliegendem Sachverhalt also bei den Hautveränderungen, höchstens zu einem Drittel, sonstige Prozesse des Betrachters (z. B. auf kognitiver Ebene) hingegen zu zwei Dritteln an der Wert- und Urteilsbildung beteiligt sind.

Physische Abweichungen wie Hauterkrankungen im Gesicht sind somit also keineswegs obligat und unvermittelt im Sinne einer direkten Reizkoppelung mit Werturteilen wie Entstellung verknüpfbar. Sie sind vielmehr indirekt als Reaktion auf Reizgegebenheiten zu sehen (Mehrabian und Blum 1997). Man kann Betrachterurteile also nicht selbsterklärend allein auf Grundlage der Stimuli erschließen, denn „verschiedene Menschen sehen und/oder bewerten physische Erscheinungen recht verschieden“ (Hünecke 2009). Diesen Ansatz unterstützen zahlreiche Studien, deren Ergebnisse nur eine vage Korrelation zwischen Attraktivitätsurteilen und visuell physischen Stimuli bescheinigen (Baker 1992; Daszkowski 2000).

\subsection{Das Schema-Konzept als Ansatz in der Entstellungsforschung}

Die recht bekannte Abbildung (Abb. 1) macht deutlich, dass bei gleichbleibender Reizgrundlage zwei unterschiedliche Sichtweisen möglich sind - die Reize werden aufgrund zweier unterschiedlicher Schemata anders verarbeitet: je nachdem, ob wir eine "junge Frau“ oder eine „alte Frau“ in dem Bild sehen, treten andere Reize in den Hintergrund (s. auch Figur-Grund-Problem in der Gestaltpsychologie). 


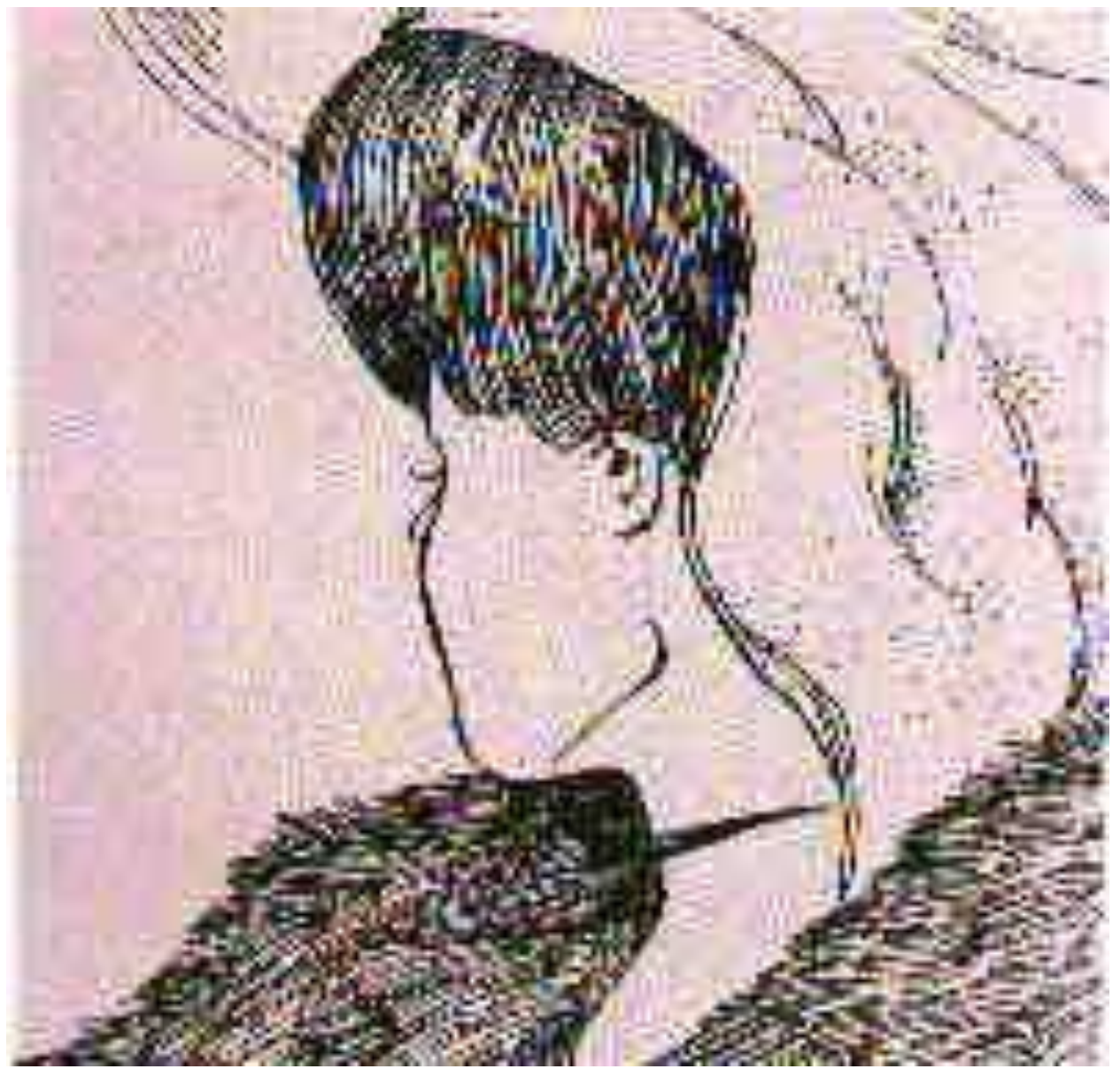

Abb. 1: Meine Frau und meine Schwiegermutter (Original: "My Wife and My MotherIn-Law") von W.E. Hill (1887-1962, Puck, 78 (2018), 11. Erstpublikation 6.11.1915; Bild abrufbar unter http://www.loc.gov/pictures/item/2010652001/)

Angesichts nachweislicher Diskrepanzen in Entstellungs-Beurteilungen muss die Frage gestellt werden, ob es vertretbar ist, noch von einem unvermittelten Konzept statt von einem vermittelnden Reiz-Reaktion-Konzept auszugehen (Hünecke 2009). Allein der Begriff „Entstellung“ setzt bereits eine, in welcher Form auch immer geartete Veränderung eines Ursprungszustandes voraus, auf den man sich gedanklich in der Wahrnehmungsbeurteilung bezieht. Ein gängiges zwischen KörperReizen und Werturteilen verknüpfendes bzw. vermittelndes Konzept ist das sogenannte Body Image (Slade 1994). Danach wird der direkte Zusammenhang von physikalischen Stimuli und Bewertungen bezweifelt. Vielmehr spielen die Eigenwahrnehmung und das Selbstbild sowie Gefühle und Gedanken eine zentrale Rolle (Cash 1997). Und dies gilt nicht nur für die sich als entstellt sehenden Patienten, sondern genauso für alle Betrachter. 
Das Schema-Konzept beinhaltet jedoch auch gewisse Annahmen. Die Wahrnehmungs-Verarbeitung ist hiernach sowohl als bottom up - als auch als top down Prozess zu sehen (Altabe und Thompson 1996; Deco und Schürmann 2000), d.h. kognitive Einflüsse wirken nicht nur auf die Informationsbeschaffung (Aufmerksamkeitssteuerung), sondern auch auf die Informationsverarbeitung (Kodieren eines Reizes).

Trifft nun ein Schema auf "fremde" inkongruente Reize, so müssen diese verarbeitet bzw. eingearbeitet werden. Diese Anpassung inkongruenter Stimuli an vorhandene Schemata kann in Form von Assimilation oder Akkommodation erfolgen. Als Assimilation wird dabei die Integration der Reize in ein bestehendes und die Erweiterung von einem bestehenden Schema bezeichnet. Unter Akkommodation wird im Sinne des Schema-Konzeptes die Schaffung eines neuen Schemas oder der Umbau eines bereits bestehenden verstanden.

Auch hier scheint das lex inertiae (Gesetz der Trägheit) eine gewisse Gültigkeit zu haben, da schemakongruente Reize schemafremden Reizen vorgezogen werden (Garcia-Marques et al. 2004; Peskin und Newell 2004).

In diesem Zusammenhang sei auf eine Studie verwiesen (Brady et al. 2005), bei der man von derselben Person Standardfotoaufnahmen und gespiegelte, seitenvertauschte Kopien verwendete. Diese wurden von der fotografierten Person selbst und von Menschen aus dem nahen Umfeld des Fotografierten bewertet. Es zeigte sich in der Auswertung, dass sich die Fotografierten auf den gespiegelten Bildern besser bewerteten, die Teilnehmer aus dem Freundes- und Bekanntenkreis aber die Standardaufnahmen signifikant besser beurteilten. Für die jeweiligen Gruppen stellten die bevorzugten Bilder die "gewohnte“, also Normalansicht bzw. Perspektive dar. Dies unterstreicht die Relevanz eigener erworbener Schemata bei der Wahrnehmung und vor allem auch Beurteilung von Reizen (vgl. Hünecke 2009).

Das „Anstarren“ könnte gemäß diesem Konzept eine, vom kognitiven Apparat geleitete, erhöhte Aufmerksamkeit für schemafremde Reize darstellen, bei der visuell erkundende Informationsbeschaffung im Vordergrund steht. Während Schemata als Konstrukt nicht direkt zu beobachten und damit zu untersuchen sind, gibt es mit dem Attribut "Aufmerksamkeit" eine messbare Größe: ein Mehr oder Weniger an Blickzeit für bestimmte Stimuli. Wie viel Aufmerksamkeit einem Reiz zukommt, hängt also von 
seiner Übereinstimmung mit bestehenden Erfahrungen (Kongruenz mit den eigenen Schemata) und der Relevanz für den Betrachter ab.

\subsection{Erfassung von Blickverhalten}

Nachdem der praktische und theoretische Hintergrund dargestellt wurde, rückt die visuell-motorische Größe des Blickverhaltens in das Interesse dieser Arbeit.

\subsubsection{Anwendungsgebiete}

Auf Grund der besonderen Bedeutung optischer Reize für den Menschen ist Blickregistrierung nicht nur Angelegenheit der medizinischen oder psychologischen Forschung. Neben diesen zwei wichtigen Feldern spielt die Blickregistrierung auch eine große Rolle in den Neurowissenschaften, der Informatik und dem wachsenden Gebiet der Mensch-Computer-Interaktion. Mittels Programmen, die durch Fixationssteuerung der Augen bedient werden, kann man beispielsweise heutzutage körperlich extrem eingeschränkten Personen die Koordination der Computermaus und damit die Computerprogrammbedienung ermöglichen. Als prominentes Beispiel kann hier Steven Hawking angeführt werden (Tollkühn 2002).

Doch auch in der Marktforschung, u.a. bei Themen wie Designing von Internetwebseiten und sogenannten „Blickfängern“, ist die Aufzeichnung von Aufmerksamkeit von besonderem Interesse. Dieser Ansatz wird daher von zahlreichen Firmen und Instituten angewandt, um beispielsweise Internetseiten auf ihre Usability, also Nutzbarkeit bzw. Benutzerfreundlichkeit, hin zu untersuchen und zu optimieren (Zerfaß und Zimmermann 2004). 


\subsubsection{Unterschiedliche Verfahren zur Blickregistrierung}

Verschiedene Verfahren können zur Aufmerksamkeitserfassung verwendet werden. In der neuesten Generation wird u. a. mit freien Systemen bearbeitet, wie z.B. dem Remote Eye-Tracker, bei dem keine den Kopf fixierenden Kinnhalterungen oder ähnliche Zusatzapparaturen benötigt werden, wie es noch bei Eye Mark Recordern oder vielen Eye-Trackern (Brüninghaus 1980) der Fall war bzw. ist. Somit kann für den Probanden ein experimentelles Szenario erzeugt werden, welches sich deutlich näher an die Realität anlehnt. Favorisiert wird im zunehmenden Maße das AttentionTracking (Broschart 2009).

\subsubsection{Die Attention-Tracking-Methode}

Bei dieser Messtechnik wird der Mauszeiger dem Fixationspunkt bzw. dem Punkt der aktuellen Aufmerksamkeit gleichgesetzt. Nicht nur die hohe Korrelation mit EyeTracking-Systemen von über 90 \% (Chen et al. 2001; Heinsen und Vogt 2003), sondern auch die Möglichkeit, mit Hilfe dieser Programme online ohne großen technischen Aufwand an einer quasi unbegrenzten Zahl verschiedener Zielgruppen testen zu können, machen dieses Verfahren zu einer sehr interessanten Alternative (Broschart 2009).

Auch diese Studie wird mit einer dieser Methode sehr ähnlich strukturierten Messtechnik durchgeführt. Dies stellt gleichzeitig eine Alternative zum Remote EyeTracker dar sowie zum früher verwandten Eye Mark Recorder (EMR), den beispielsweise Brüninghaus (1980) bei seiner Untersuchung über Hautveränderungen und Blickverhalten nutzte.

\subsection{Fragestellung}

Anliegen dieser Arbeit ist die Untersuchung des Blickverhaltens von Probanden mit Hilfe des Restricted Focus Viewer (RFV). Betrachtet werden jeweils ein hautgesundes $(\mathrm{Hg})$ und ein identisches hautkrankes $(\mathrm{Hk})$ Portrait-Bild derselben Person, wobei die Bilder verschiedenen Bewertungsgruppen zugeordnet sind (attraktiv, nicht attraktiv bzw. stark verändert, leicht verändert). Die Betrachter haben in der Unter- 
suchung die Aufgabe, die Portraitbilder anhand verschiedener Attribute (Attraktivität, Auffälligkeit, Angenehmheit, Ungewöhnlichkeit) zu beurteilen. Verschiedene methodische und inhaltliche Problemstellungen sollen damit verfolgt werden.

Es wurden diesbezüglich folgende Thesen und Hypothesen aufgestellt:

1. Das RFV-Verfahren erweist sich als brauchbarer methodischer Ansatz zur Untersuchung von Betrachtungen eines Gesichts einschließlich von Hautveränderungen (z.B. Eignungsaspekte: Probanden können mit dem RFV-Verfahren arbeiten; es zeigt sich ein ähnliches Betrachtungsverhalten, wie es beispielsweise aus Studien mit Eye-Movement-Recordern zu erwarten ist [z.B. Hitliste der Gesichtsmerkmale mit Dominanz der Augen]; Luria und Strauss 1978).

2. Hautveränderungen im Gesicht sind theoretisch als eine Abweichung vom Betrachtungsschema "Gesicht" anzusehen. Danach ist zu erwarten, dass diese Hautveränderungen eine erhöhte Aufmerksamkeit bewirken (d.h. signifikant höhere Betrachtungszeit für ein Stigma-Areal). Weiterhin führt im Sinne des Primings eines Schemas ein vorher gezeigtes hautkrankes Bild bei dem nachfolgenden hautgesunden Bild zu einer Art „Erwartungshaltung“ für einen nun nicht mehr vorhandenen Reiz (früher vorhandene Hautveränderung).

3. Es bestehen unterschiedliche Zusammenhänge zwischen Aufmerksamkeit/Blickverhalten und emotionalen bzw. kognitiven Bewertungen:

a. Es gibt nur einen sehr kleinen oder gar keinen Zusammenhang zwischen der Aufmerksamkeit für Hautveränderungen und der Attraktivitätseinstufung (emotionale Bewertung).

b. Es gibt einen bedeutenden Zusammenhang zwischen der Aufmerksamkeit für Hautveränderungen und der Ungewohntheitseinstufung (kognitive Bewertung).

c. Der Zusammenhang zwischen der Fixationszeit und kognitiver Bewertung fällt signifikant größer aus als der Zusammenhang von Fixationszeit und emotionaler Bewertung. 


\section{Material, Methoden und Durchführung}

In diesem Kapitel wird beschrieben, wie bei der Untersuchung methodisch vorgegangen wurde. Dazu wird die Zusammensetzung von Portraitfotos und Probandinnen erläutert, bevor eine ausführliche Darstellung der Funktionsweise des RFV erfolgt. Abschließend wird beschrieben, wie die mittels RFV-Technik gewonnenen Daten ausgewertet wurden.

\subsection{Reizstimuli: Auswahl der Portraitfotos}

Der Schwerpunkt der Untersuchung lag auf dem Körperareal „Gesicht". Grundlage dafür sind zahlreiche Vorgängerstudien, die wiederholt die enorme Bedeutung des Gesichts für zwischenmenschliche Interaktionen herausstellten (z.B. Fridlund 1991; Öhman 1993; Aharon et al. 2001; Ekman 2003; Fischer et al. 2004; Hall et al. 2005). Die Konzentration der Aufmerksamkeit auf das Gesicht hängt jedoch nicht nur mit evolutionären oder reproduktiven Vorteilen und Aufgaben zusammen (Thornhill und Grammer 1999; Buss 2004). Es kann vielmehr schon im frühen Kleinkind- und Kindesalter ein verstärktes Interesse für Gesichter und Reaktionen auf unterschiedliche Gesichtsausdrücke beobachtet werden (Johnson et al. 1991; Morton und Johnson 1991). Auch Hewig et al. (2008) kamen in ihrer Studie zu vergleichbaren Ergebnissen. Sie untersuchten geschlechtsspezifische Unterschiede zwischen Männern und Frauen im Betrachtungsverhalten von Ganzkörperfotos und konstatierten, dass unabhängig von Geschlecht und präsentiertem Foto, das Gesicht die mit Abstand größte Aufmerksamkeit erhielt. In Einklang mit diesen Erkenntnissen konnte auch mit objektiven bildgebenden Verfahren, z.B. mit dem MRT, demonstriert werden, wie hoch spezialisiert das menschliche Gehirn für Reize und Informationen aus dem Gesicht ist (Hoffman und Haxby 2000; Haxby et al. 2001; Schweinberger et al. 2004).

Das Bildmaterial umfasste 16 Portraitfotos, die jeweils einmal als hautgesund und einmal als hautkrank in der Größe 600x900 Pixel präsentiert wurden. Insgesamt sind von den Probandinnen demnach 32 Fotos beurteilt worden.

Alle ausgewählten Portraitfotos mussten verschiedene Anforderungen erfüllen. Personen auf den Portraitfotos sollten einen möglichst neutralen Gesichtsausdruck 
(ohne besondere Mimik) aufweisen, keine Brillen- oder Kontaktlinsenträger sein und nicht schielen. Darüber hinaus sollte auf den Bildern nach Möglichkeit nur das Gesicht mit Hals und Haaren abgebildet sein. Zusätzlich waren alle hier verwendeten Fotos en-face-Bilder oder leicht davon abweichend bis hin zum Halbprofil, um die Sichtbarkeit beider Augen sicherzustellen.

Ein weiteres Zielkriterium stellte die Abgrenzbarkeit der Hautveränderungen auf den Portraitfotos dar. Einerseits sollten sich also die Hautveränderungen farblich von der „normalen“, gesunden Haut absetzen. Andererseits sollten sie an Stellen liegen, welche im Allgemeinen nicht so viel Aufmerksamkeit erhalten (z.B. Stirn, Kinn, Wange) wie die Merkmale bei der „typischen“ Betrachtung von Gesichtern (z.B. Augen, Nase, Mund) (Luria und Strauss 1978). Indem nun besonders in den normalerweise eher „vernachlässigten“ Arealen ein signifikanter Aufmerksamkeitsschub feststellbar sein sollte, würde am einfachsten der Nachweis gewährleistet werden, ob überhaupt Hautveränderungen die Aufmerksamkeit leiten.

Von allen Fotos lag vorab jeweils nur eine hautgesunde oder eine hautveränderte Version vor. Mithilfe eines Bildbearbeitungsprogramms (ACDSee 2.5) wurde ein identisches Pendant geschaffen, indem entweder eine Hautveränderung gezielt eingebaut- oder die bereits vorhandene Hautveränderung retuschiert wurde. Nach diesem Arbeitsschritt gab es also immer ein Fotopaar, welches bis auf das Hautkrankheitsmerkmal exakt übereinstimmte. Außerdem konnten so auch andere Zielkriterien, wie beispielsweise die Untersuchung von Hautveränderungen die außerhalb der Merkmale Augen, Nase und Mund liegen, sowie eine Variation des Ausmaßes der Hautveränderung (Größe und Farbintensität), erfüllt werden.

Die Mehrheit der 16 Basis-Bilder $(n=14)$ stammte aus dem Foto-Archiv der Hautklinik der Universitätsmedizin Göttingen. Zwei weitere Portraitbilder sind aus Foto-Foren des Internets entnommen worden.

Die Portraitfotos wurden so ausgesucht und zusammengestellt, dass die untersuchten vier verschiedenen Gruppen in gleicher Häufigkeit besetzt waren. Jede Gruppe umfasste demnach vier Bilder:

1. eher attraktiv _ $\quad$ leichte Hautveränderung

2. eher attraktiv _ $\quad$ starke Hautveränderung 
$\begin{array}{llll}\text { 3. } & \text { weniger attraktiv } & - & \text { leichte Hautveränderung } \\ \text { 4. } \quad \text { weniger attraktiv } & - & \text { starke Hautveränderung }\end{array}$

Dadurch sollte das Konfundieren eines Merkmals verhindert werden und gleichzeitig gewährleistet sein, dass das Ausmaß der Hautveränderung und der Attraktivität über alle verwendeten Fotos hinweg ausbalanciert war.

Die Zuteilung zu den jeweiligen Gruppen erfolgte unter Berücksichtigung einer im Voraus durchgeführten Bilderbewertung. Dabei wurden die Fotos von einer dem Untersuchungskollektiv ähnlichen Gruppe $(n=10)$ aus dem Bekanntenkreis des Versuchsleiters auf einer Skala von 0-10 benotet. In einem ersten Durchgang ist die Attraktivität der Personen auf hautgesunden Bildern $(\mathrm{Hg})$ bewertet worden und in einem zweiten Teil wurde der Schweregrad der hautkranken Veränderungen auf den entsprechenden Portraitbildern (Hk) beurteilt.

\subsection{Stichprobe der Versuchspersonen}

Um eine hohe Relevanz für die Probanden in der ganzen Untersuchung zu erzielen, wurde neben den Portraitfotos auch das Probandenkollektiv in Geschlecht und Alter eingeschränkt. Hewig et al. (2008) konnten zeigen, dass es signifikante Unterschiede im Betrachtungsverhalten von männlichen und weiblichen Personen gibt. Diese intergeschlechtlichen Differenzen beziehen sich jedoch nicht nur auf das Betrachtungsverhalten der beiden Gruppen an sich, sondern variieren darüber hinaus mit dem Geschlecht, das betrachtet wird (z.B. Männer schauen Frauen anders an als inre Geschlechtsgenossen und umgekehrt). Darüber hinaus fanden Hewig et al. (2008) heraus, dass es im Gegensatz zur männlichen Gruppe bei den Frauen keine systematischen Korrelationen zwischen ihren subjektiven Bildbewertungen und dem Blickverhalten gab.

Um diese nach aktuellem Stand der Forschung differenzierte Problemlage zu vereinfachen, wurde die Untersuchung auf Frauen zwischen 15 und 28 Jahren beschränkt, denen ausschließlich Portraitfotos weiblicher Personen in der gleichen Altersspanne präsentiert worden sind.

Bei den Probandinnen gab es keinerlei Einschränkungen hinsichtlich des Bildungsund Ausbildungsgrades. Es nahmen junge Frauen mit Haupt-, Realschulabschluss 
und Abitur teil. Einige besuchten noch die Schule, andere waren bereits in der Ausbildung, besuchten eine Universität oder waren schon berufstätig. Insgesamt betrug die Anzahl der Teilnehmerinnen 40 Personen.

\subsection{Untersuchungsmethode: der Restricted Focus Viewer (RFV)}

Wie zu Beginn des Kapitels erwähnt, soll an dieser Stelle detailliert auf die Funktionsweise der in dieser Studie verwendeten Methode zur Erfassung des Blickverhaltens eingegangen werden. Aufgrund der zentralen Rolle für diese Arbeit und der Frage nach möglichen Einsatzgebieten, werden an dieser Stelle auch relevante Ergebnisse aktueller und zurückliegender Forschung eingehender dargestellt.

\subsubsection{Funktion und Anwendungsgebiet}

Das Kernstück der experimentellen Aufmerksamkeitserfassung war der Restricted Focus Viewer (RFV) (Blackwell et al. 2000). Wie der Name schon impliziert, arbeitet dieses auf der Computersoftware JAVA basierende Programm mit einem eingeschränkten Sichtfeld. Es ist für den Probanden nur ein Ausschnitt des Portraitfotos klar und deutlich und in dieser Untersuchung „farbrichtig“ sichtbar. Dieser Ausschnitt kann mit der Maus über das Foto hinweg bewegt und gesteuert werden. Der Rest des Fotos ist nicht unsichtbar, sondern in Form eines Fotonegatives "undeutlich“ gemacht (Luria und Strauss 1978). Um einen Eindruck von der Ansicht der Probanden zu bekommen, werden hier einige Beispielfotos aus der vorangeschalteten Übungsphase gezeigt. 

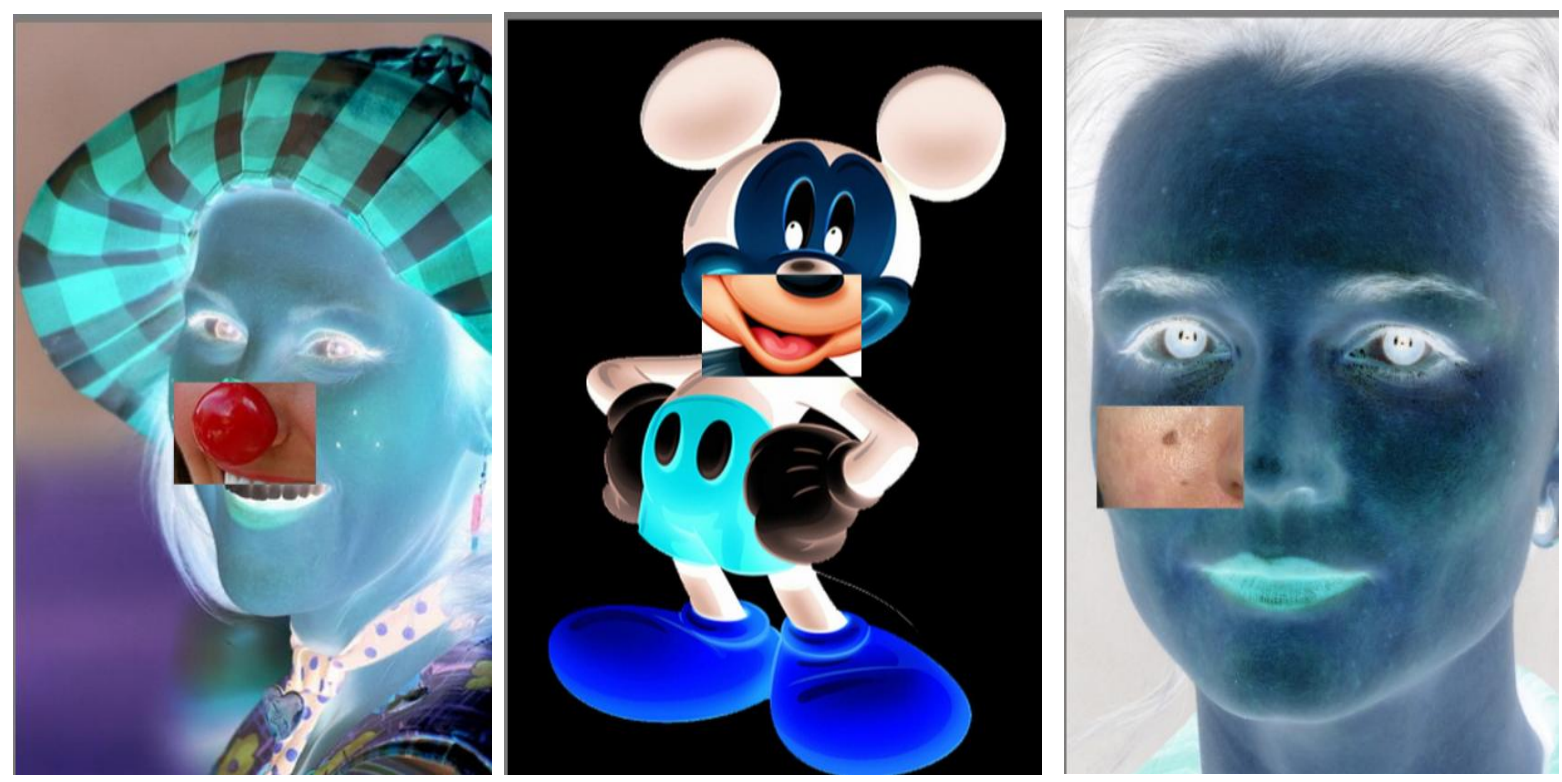

Abb. 2: Bilder aus der Übungsreihe der Einleitung. Wie zu erkennen ist, kann nur der Ausschnitt des Fokus-Fensters "farbgetreu“ beurteilt werden, während der restliche Bildausschnitt als „Fotonegativ" präsentiert wird. 
In der ursprünglichen RFV-Version ist der nicht im Fokus-Fenster befindliche Bildausschnitt lediglich mit einem starken Weichzeichner mehr oder weniger stark „verschleiert" worden.
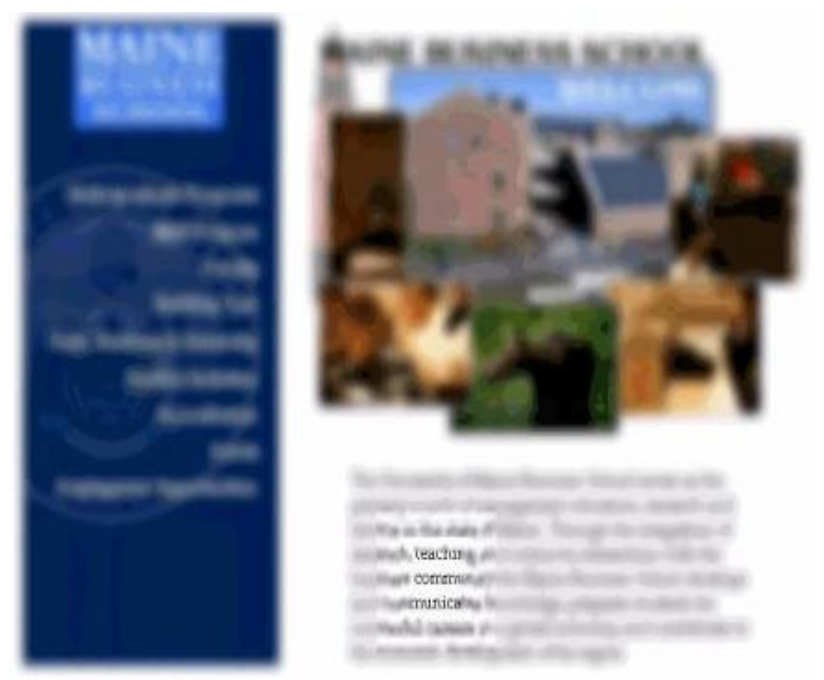

Abb. 3: Die ursprüngliche Variante des RFV lässt Strukturen außerhalb des FokusFensters (im unteren Textbereich zu sehen) „verschwimmen“, präsentiert sie allerdings in originaler Farbe.

Weitere Argumente für die Verwendung der Negativ-Technik des „Verschleierns“ von nicht-fokussierten Bildanteilen werden in Abschnitt 2.3.3 genauer dargestellt.

Durch Nutzung des RFV und der Negativ-Technik sollte dem natürlichen menschlichen Sehen bestmöglich entsprochen werden: Der Ort des schärfsten Sehens, der beim Menschen in der Fovea lokalisiert ist (Grehn 2008a), sollte sein Pendant im Fensterausschnitt haben. Nur in diesem Ausschnitt kann das Bild hochauflösend in Echt-Farbe und in allen Einzelheiten realistisch betrachtet werden.

Dieses als Focus-Window-Technique (Jones und Mewhort 2004) bezeichnete Verfahren ermöglicht Aussagen zur Aufmerksamkeit und Aufmerksamkeitssteuerung durch Übereinstimmung der visuellen Aufmerksamkeit mit dem Fokus-Fenster und dessen Steuerung mit der Mausbewegung. Beispielhaft sind an dieser Stelle zwei der Übungsbilder nach einer 30-sekündigen Betrachtung jeweils in Scanpath- bzw. Heatmap-Technik (Erläuterung siehe Abschnitt 2.5) wiedergegeben: 

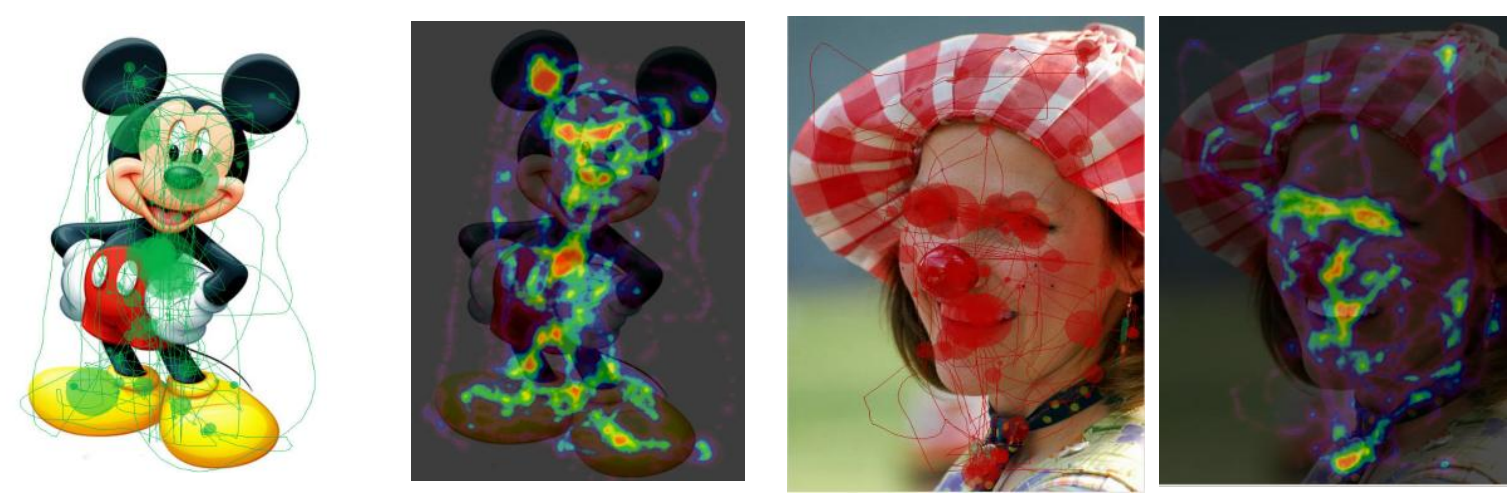

Abb. 4: Auswertungsmöglichkeiten der RFV-Daten. Analyse der verschiedenen Bewegungsmuster und Verweildauern des Fokus-Fenster (Darstellung des Scanpath jeweils links und der Heatmap rechts).

\subsubsection{Zur allgemeinen Validität des RFV}

Die Validität der RFV-Technik prüften u.a. Blackwell et al. (2000). Sie führten einen Vergleich zur Aufmerksamkeitserfassung zwischen Eye-Tracking-Systemen und dem Restricted Focus Viewer durch. Ihr Anliegen bestand darin, Gemeinsamkeiten in der Suchstrategie, also der Beschaffung von navigatorischer und detaillierter Information, nachzuweisen. Dazu wurden den Probanden Fotos in Kombination mit einer Aussage zur Abbildung gezeigt. Den Wahrheitsgehalt der Aussage konnten die Versuchspersonen nur durch sorgfältige Betrachtung des Fotos überprüfen. Die Aufmerksamkeit wurde dabei einmal in Form der Augenbewegungen mit dem EyeTracking-System und einmal in Form der Fensterbewegungen des Restricted Focus Viewer aufgezeichnet.

In der Auswertung ergaben sich keine signifikanten Unterschiede im Bereich der Durchmusterungsstrategien zwischen den beiden Systemen. Die mit dem EyeTracking-Verfahren erzielten Resultate ließen sich mit der RFV-Technik replizieren. Somit kann davon ausgegangen werden, dass der Restricted Focus Viewer ein gleichwertiges Verfahren zur Aufmerksamkeitserfassung ist.

Weiterhin belegt eine Untersuchung von Chen et al. 2001, dass der Restricted Focus Viewer eine starke Korrelation zwischen dem Fixationspunkt der Probanden und der Position des Mauszeigers bzw. des Fokus-Fensters aufweist. Daher kann davon ausgegangen werden, dass die Position des Fensters auch mit dem tatsächlichen Fixationspunkt des Betrachters weitestgehend übereinstimmt. Indem der RFV den 
Verlauf und die Betrachtungszeiten des Fensters an allen Orten und zu jedem Zeitpunkt registriert, können anhand dieser Daten Aussagen über die Aufmerksamkeit, Aufmerksamkeitssteuerung und Aufmerksamkeitsveränderung gemacht werden.

Darüber hinaus bietet der RFV einige Vorteile. So ist die Durchführung von Testabläufen nicht nur apparativ weniger aufwendig, sondern auch flexibler, z.B. im Bezug auf den Untersuchungsort, durchführbar. Ein nicht unbeträchtlicher Teil des Probandenkollektivs wäre schon auf Grund des Tragens einer Brille für einen Test mit Eye-Tracking-Systemen gar nicht in Frage gekommen. Solche Ausschlusskriterien schränken die Auswahl an Probanden und dadurch ggf. auch die Aussagekraft der Ergebnisse ungewollt ein.

\subsubsection{Filterung der Bildvorlagen - Trennung diagnostischer und navigatorischer Informationen}

Wie zuvor bereits gezeigt, werden die Areale außerhalb des Fokus-Fensters durch einen "Filter" unkenntlich gemacht. Ziel dieser Filterung ist es, den Betrachtern möglichst viel navigatorische und gleichzeitig möglichst wenig diagnostische Information außerhalb des Fensters zu geben (Jones und Mewhort 2004). Mit navigatorischer Information ist dabei die Information gemeint, welche den Probanden z.B. Aufschluss über jene Lokalisationen gibt, an denen sich potentiell wichtige Detailinformationen (diagnostische Information) befinden könnten. Als diagnostische Information bezeichnet man dabei die Information, welche relevant für die Bearbeitung der Fragestellung ist.

In dieser Studie sollte es den Betrachtern möglich sein, trotz des Filters nicht nur die Lokalisationen der wesentlichen Gesichtsmerkmale wie Auge, Nase und Mund, sondern auch die möglicher anderer Gesichtsdetails, wie beispielsweise von Hautveränderungen, realisieren zu können, um diese mit dem Fenster anzusteuern und dann detailliert zu betrachten.

Diagnostische Information wird dem Probanden bei der visuellen Restriktion mit dem RFV fast nur innerhalb des Mausfensters zugänglich gemacht. Außerhalb des Fensterausschnitts wird sie mittels Filterung minimiert, gleichzeitig jedoch der größtmögliche Gehalt an struktureller Information zugelassen, damit die diagnos- 
tische Information überhaupt angesteuert werden kann (navigatorische Information, vgl. Beispielfotos in Abschnitt 2.3.1).

Anders als bislang kam bei der RFV-Technik ein Negativ-Filter (vgl. Luria und Strauss 1978) statt eines Weichzeichner-Filters zum Einsatz. Durch die Verwendung von Fotonegativen kann es jedoch zu anderen Schwierigkeiten kommen. Denn bei der Negativmethode ist vermutlich mit einer Farbverschiebung für den fotografischen Laien kein so deutlicher Hinweis auf eine Merkmalsänderung gegeben, wie bei dem Schwarz-Weiß-Wechsel von Konturlinien (z.B. bei Erythemen im Gegensatz zu Grenzlinien eines Nävus). Um hier keinen zu großen Unterschied im StimulusMaterial zuzulassen, sind deshalb mittels Bildbearbeitung die Rot-Grün-Bereiche beim Negativ im Kontrast verstärkt worden.

\subsubsection{Motion-Blur-Technik beim RFV}

Ein weiterer Aspekt bei der Nachbildung des menschlichen Sehvermögens ist das begrenzte Auflösungs- und Verarbeitungsvermögen während sehr schneller Augenbewegungen, den Sakkaden (Grehn 2008b). Im RFV werden solche Sakkaden durch den Motion-Blur-Speed simuliert. Diese Motion-Blur-Speed genannte Technik führt beim Überschreiten einer definierten Geschwindigkeit bei der Bewegung mit dem Navigationsfenster zu einer Art Flimmern. Wie bei Sakkaden im menschlichen Sehvermögen kann so auch der Proband bei sehr schnellen Positionsänderungen des Mausfensters im RFV nicht gleichzeitig fixieren. Dies soll einerseits verhindern, dass zickzack- oder kreisende Bewegungen zu einer Art vergrößertem Fenster verwendet werden können und anderseits eine Unterscheidung zwischen ausgeführten Bewegungen und wirklichen Fixationen ermöglichen (Jansen 2001), da nur dann scharf gesehen werden kann, wenn der Blick, also das Fenster als RFVÄquivalent, zumindest kurzzeitig „ruht“.

Zusätzlich bekamen die Probanden in der Einleitung die Instruktion, alle Bilder „eingehend“ zu betrachten. 


\subsubsection{Das Focus-Fenster des RFV}

Die nächste Überlegung galt der Festlegung eines Größenbereichs des potentiellen Fixationsareals. Beim RFV-Verfahren entspricht dies der Größenbestimmung des Fokus-Fensters. Bei der Auswahl gab es mehrere Faktoren zu berücksichtigen. Zunächst einmal handelte es sich bei den Untersuchungsfotos ausschließlich um Gesichter. Die Aufgabenstellung erforderte eine umfassende, aber detaillierte Betrachtung der Bilder. Bei der Auswahl der Fenstergröße musste berücksichtigt werden, dass nach Möglichkeit jeweils nur ein diagnostisches Merkmal erfasst wurde und nicht mehrere simultan eingefangen werden konnten. Auf die Arbeit von Kahle (2007) stützend und nach mehreren Simulationen, wurde die Größe des FokusFensters mit 175 mal 120 Pixeln festgelegt, bei einer Portraitbildgröße von 600 mal 900 Pixeln.

\subsection{Durchführung}

Die Durchführung der Untersuchung erfolgte mit einem Laptop der Marke „ACER“. Verwendet wurde das Modell „TravelMate 8103WLMi“ mit einer Bildschirmgröße von 15.4" und einer Auflösung von 1680 × 1050 Pixel. Da die Größe der Fotos mit 600x900 Pixeln deutlich darunter lag, wurde für deren Hintergrund während der Präsentation ein neutraler Grauton gewählt. Mit dem Laptop ließ sich der Untersuchungsort flexibel gestalten, sodass es keine Beschränkung auf den Umkreis Göttingen gab. Die Probandinnen (zur genauen Zusammenstellung der Teilnehmerinnen siehe Abschnitt 2.2) wurden jeweils einzeln in einem ruhigen Raum getestet. So sollte eine weitestgehend ungestörte Atmosphäre für die Bearbeitung der Fotos gewährleistet werden (geräuscharm und geringer Lichteinfall).

Als Vorinformation war den Versuchspersonen lediglich bekannt, dass es um das Betrachten und die Bewertung von Bildern gehen würde. Gestartet wurde nicht sofort mit einem der 32 Fotos, sondern mit einer kurzen Instruktion, um die Probandinnen mit der Technik vertraut zu machen. Dazu bearbeiteten sie zunächst drei verschiedene Übungsbilder (Beispiele s.o. Abb. 4). Diese wurden, wie auch später die Testbilder, für 30 Sekunden präsentiert. Das Verkürzen oder Verlängern dieser Zeitspanne sowie ein wiederholtes Betrachten der Fotos war ausgeschlossen. Zusätzlich gab es im Voraus und im Nachhinein Erläuterungen zum Umgang mit dem 
Mausfenster und zur Beantwortung der verschiedenen Fragen, die im Anschluss an die Bildpräsentation gestellt wurden. Eine zielgerichtete, selbstständige Bearbeitung der Fragestellungen zu den 32 Versuchsbildern sollte so ermöglicht werden.

Die Anwesenheit des Versuchsleiters beschränkte sich auf diesen Instruktionsteil. Am Ende der Einleitung wurde sowohl in schriftlicher als auch in mündlicher Form nach verbliebenen Unklarheiten gefragt, um diese dann gegebenenfalls noch vor Präsentationsbeginn der Versuchsfotos auszuräumen.

Die eigentliche Bearbeitung der 32 Experimentalbilder führten die Probandinnen dann selbstständig ohne Anwesenheit dritter Personen aus. Damit sollte u.a. vermieden werden, dass sich die Teilnehmerinnen während der Betrachtung der Portraitfotos und Beantwortung der Fragen beobachtet fühlten und dadurch Faktoren wie soziale Erwünschtheit (political correctness) die Ergebnisse beeinflussen.

Nach einer 30-sekündigen Präsentation jedes Experimentalfotos wurden die Versuchsteilnehmerinnen aufgefordert, das Bild anhand verschiedener Eigenschaften einzuschätzen. Dies erfolgte anhand der Attribute Attraktivität, Auffälligkeit, Angenehmheit und Ungewöhnlichkeit, die bereits in früheren Untersuchungen die Erfassung unabhängiger Dimensionen ermöglichten (vgl. Kahle 2007). Für die FotoBewertung selbst bestand kein Zeitlimit. Es wurde jedoch ausdrücklich darauf hingewiesen, diese möglichst ohne lange Überlegungen an die Betrachtung anzuschließen.

Zur Einstufung der Eigenschaften diente den Probandinnen eine zehnstufige Bewertungsskala. Jedes Experimentalfoto wurde also direkt nach der Bildpräsentation in Bezug auf die vier Attribute von "“ (nicht attraktiv, nicht auffällig, nicht angenehm, nicht ungewöhnlich) bis "10“ (sehr attraktiv, sehr auffällig, sehr angenehm, sehr ungewöhnlich) eingeschätzt.

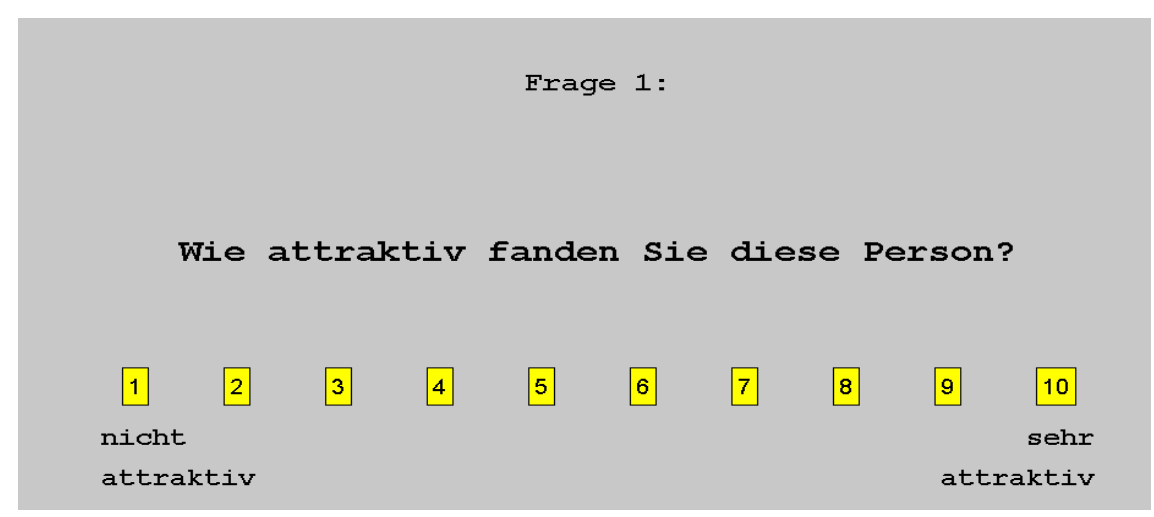

Abb. 5: Beispiel für die Ansicht einer Bewertungsskala, hier Attraktivitätseinstufung. 
Diese Werte wurden, ebenso wie die Fixations- und Navigationsbewegungen des Mausfensters, vom Restricted-Focus-Viewer-Programm erfasst. So lagen am Ende zu jedem Bild neben den zeitlichen und räumlichen Daten vier entsprechende Skalenwerte zur Einschätzung der Attribute vor.

Eine kurze Einführung in ein „soziales Szenario“ wurde zwischen der Instruktion und dem Start der Experimentalfotoreihe präsentiert. Zielsetzung dabei war es, die Versuchsteilnehmerinnen gedanklich in eine möglichst reale Lebenssituation zu versetzen oder gegebenenfalls wieder zurückzuversetzen. Dazu wurde eine kleine Bilderfolge eines Stadtbummels verwendet, verbunden mit dem Besuch eines Cafés, wobei innen die nachfolgend gezeigten Personen begegnet sein können.
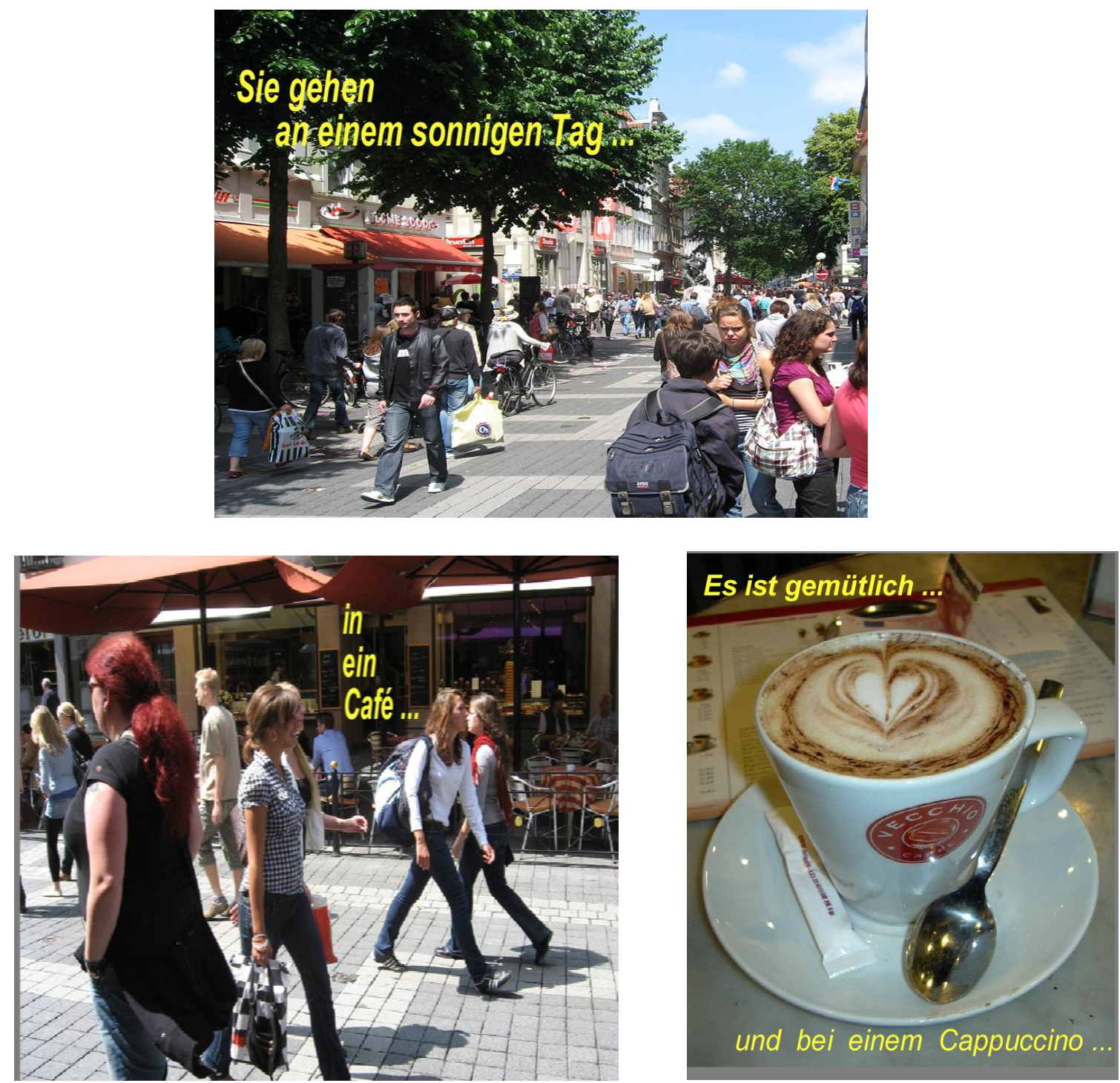

Abb. 6: Fotosequenz „Straßencafé“. 
Dieser Rahmen der Präsentation war für alle Testdurchläufe identisch. Mit variierenden Kommentaren zwischen den Bewertungen und dem darauf folgenden Foto, sollte die Gefahr aufkommender Monotonie reduziert werden.

Darüber hinaus wurde nach der ersten Hälfte der Untersuchung, also nach Betrachtung der anfänglichen 16 Fotos, erneut eine kurze "Café-Bilderabfolge“ präsentiert, um die Testkandidatinnen wie bereits erläutert möglichst gedanklich in diesem Szenario zu halten.

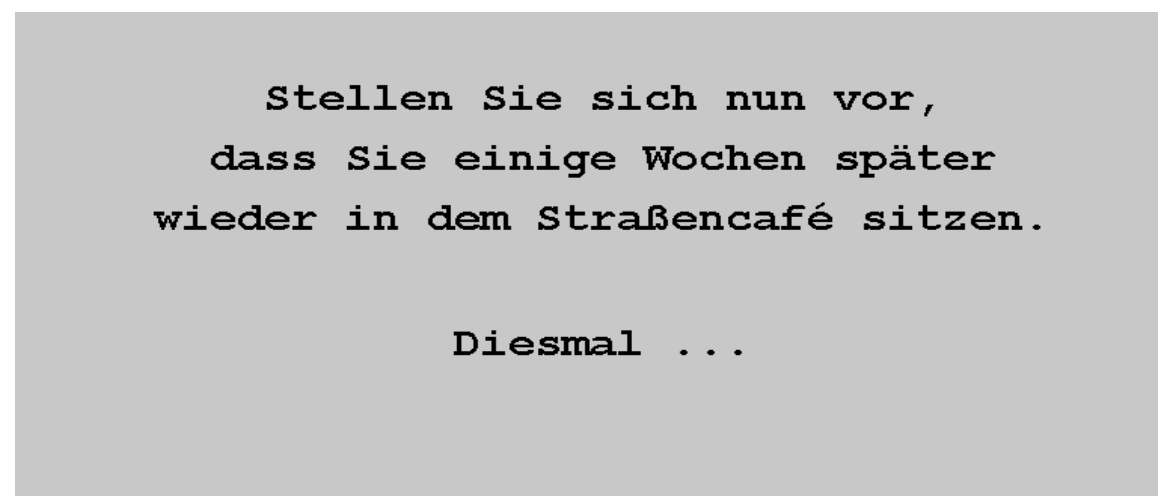

Abb. 7: Zwischensequenz nach 16 Portraitfotos.

Nach Bearbeitung der verbliebenen 16 Bilder wurden den Versuchsteilnehmerinnen Fragen zur Evaluierung des Versuchs und zur eigenen Person gestellt (der Fragenkatalog ist dem Anhang beigefügt). Die Ergebnisse der Evaluation werden in Abschnitt 4.1 besprochen.

Bei der Versuchsdurchführung wurden vier verschiedene Versionen der RFV-Dateien verwendet. Der Unterschied bestand in der Permutation der Reihenfolge, in welcher die Experimentalfotos den Probandinnen präsentiert wurden, um mögliche Einflüsse der Präsentationsreihenfolge der einzelnen Bilder als Störvariable bezüglich des Betrachtungs- oder Bewertungsverhaltens auszugleichen (Präsentationsschema siehe Anhang). Alle vier Versionen sind von jeweils 10 Teilnehmerinnen bearbeitet worden, deren Gruppenzuordnung zufällig erfolgte. 


\subsection{Auswertung}

Die über das RFV-Verfahren gewonnenen Dateien mussten für die weitere deskriptive und statistische Auswertung aufbereitet werden. Dazu wurde in der Programmiersprache für das SAS (Statistical Analysis System) -Programmpaket ein jeweils angepasstes Programm geschrieben.

Für die rein deskriptiv-bildliche Darstellung des Betrachtungsverhaltens in Form von Scanpath- und Heatmap-Bildern (siehe Abb. 4) stand das AAT (Attention-AnalysisTool) von Neumann (2007) zur Verfügung. Damit kann gut veranschaulicht werden, wohin und für wie lange auf ein Bild geschaut wird. Beim Scanpath-Ansatz wird die Fixationslänge durch einen Kreis dargestellt, dessen Größe mit der Dauer der Betrachtungszeit korreliert. Beim Heatmap-Ansatz wird die Fixationsdauer durch Farbe und Transparenz wiedergegeben: Je länger eine Position betrachtet worden ist, desto mehr wandelt sich die Farbe von Blau zu Orange und gleichzeitig von opak zu transparent. Der Scanpath-Ansatz macht deutlich, in welcher Reihenfolge verschiedene Fixationspunkte angesteuert worden sind. Dadurch scheinen sie, grafisch dargestellt, wie ein „Spinnennetz“ miteinander verbunden zu sein.

Für die statistische Auswertung wurden unterschiedliche SAS-Programme genutzt. Dafür musste allerdings zuvor eine spezielle Datenaufbereitung durchgeführt werden: Aus den kontinuierlichen Zeit- und Koordinatenwerten (etwa 1000 Datenerhebungen pro Bild-Betrachtung) mussten summarische Werte gebildet werden. Zu diesem Zweck wurden die einzelnen Portraitbilder in Felder aufgeteilt: Fielen nun Zeit- und Koordinatenwerte in diese Felder, dann führte das zu einer Erhöhung von (summarischen) Fixationszeiten und -häufigkeiten für dieses Feld (s. entsprechendes Auswertungsverfahren bereits früher bei Brüninghaus [1980]). Dank der deskriptivbildlichen Ergebnisdarstellung über Scanpath- und Heatmap-Bilder konnte die Felderaufteilung für die Portraits nicht nur theoretisch festgelegt, sondern auch empirisch optimiert werden. 


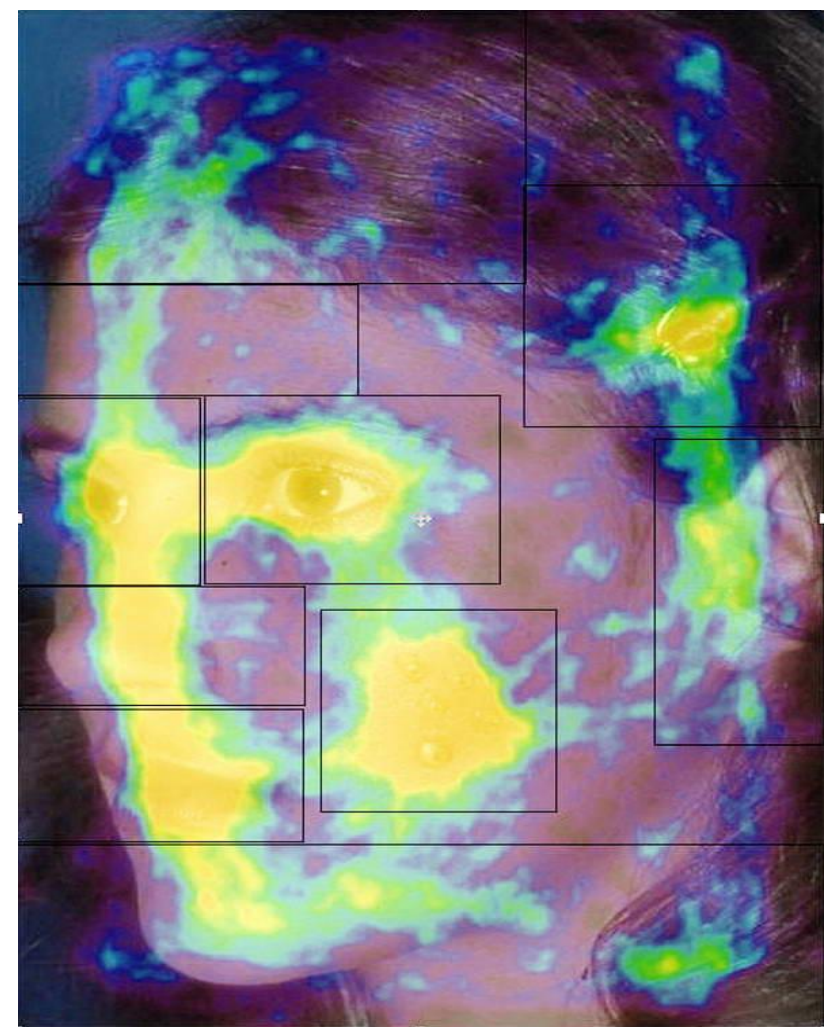

Abb. 8: Beispielhafte Feldaufteilung der Fixationsbereiche (empirisch optimiert durch Heatmap-Darstellung, welche die Betrachtungszeiten aller Probandinnen aufsummiert).

Im Gegensatz zur sonst schematisch genutzten R-Technik wurden die Korrelationen zwischen den Fixationszeiten für Stigma-Areale und den dazugehörigen Bewertungen nach der P-Technik berechnet, d.h. für jeweils einen Probanden ist über die entsprechende Anzahl von Bildern hinweg die Beziehung zwischen Fixation und Bewertung errechnet worden. 


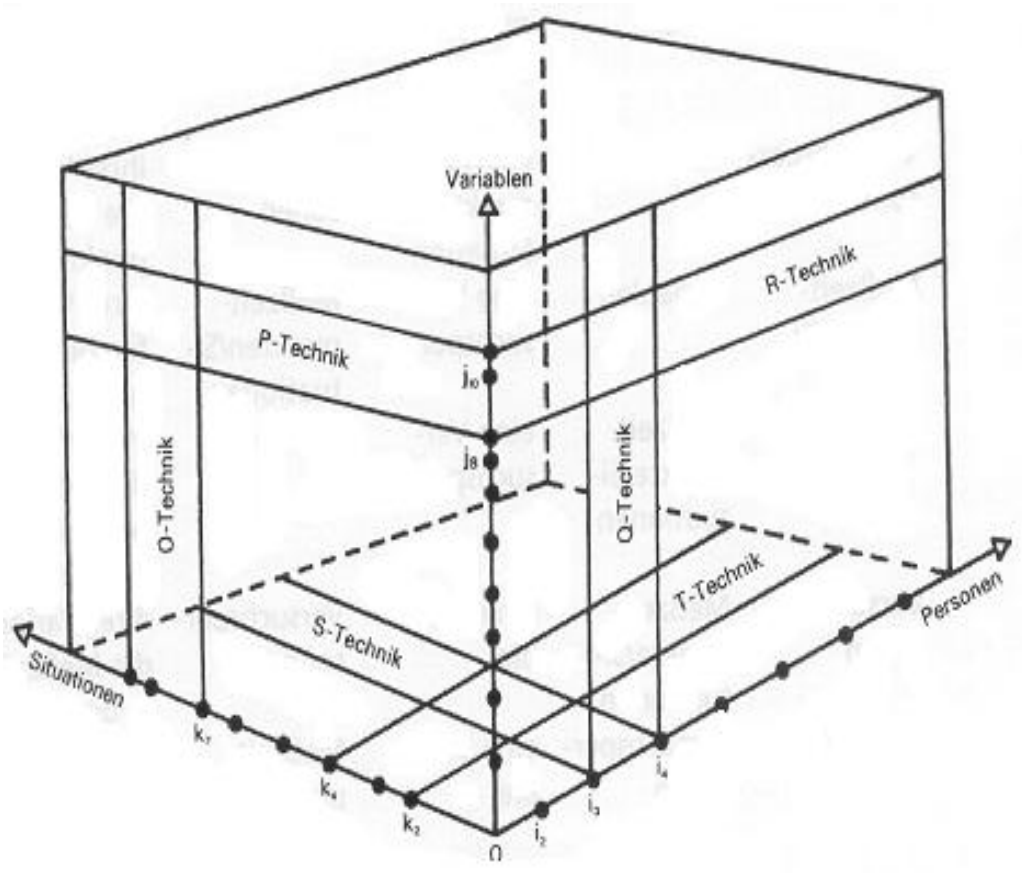

Abb. 9: Datenquader von Cattell (1946). Die P-Technik ist der Vergleich von Merkmalen über Situationen unter „Ausblendung“ der Person. 


\section{Darstellung der Ergebnisse}

In diesem Kapitel werden die gewonnenen Ergebnisse der Untersuchung über die hautgesunden und hautkranken Portraitbilder vorgestellt, insbesondere in Bezug auf die zuvor aufgestellten Thesen und Hypothesen.

\subsection{Prüfung der unabhängigen Variablen und thematisch- relevante Selbsteinschätzung}

Als unabhängige Variable wurden in die Untersuchung "Attraktivität" und „Schweregrad der Hautveränderung" eingeführt. Beide Variablen sollten zudem auch untereinander möglichst unabhängig voneinander sein. Vorab wurde ermittelt, ob auch das Untersuchungskollektiv zu ähnlichen Bewertungen kommt. Dazu dienten - $\quad$ zum einen die Attraktivitäts-Urteile, welche die 20 Probandinnen abgegeben haben, als sie zum allerersten Mal eins der 16 Portraitfotos gesehen hatten - und zwar in der hautgesunden Variante

- $\quad$ zum anderen die Schweregrad-Urteile, die je 10 Probandinnen am Versuchsende zur Kontrolle abgegeben hatten - und zwar sind dort noch einmal jeweils 4 Bilder bewerten worden (gemäß der Vorklassifikation aus jeder Bild-Gruppe je 1). Wie Abb. 10 zeigt, streuen, wie angestrebt, Attraktivitäts-Werte und Schwere-Werte der 16 Bilder weitestgehend unabhängig voneinander. 


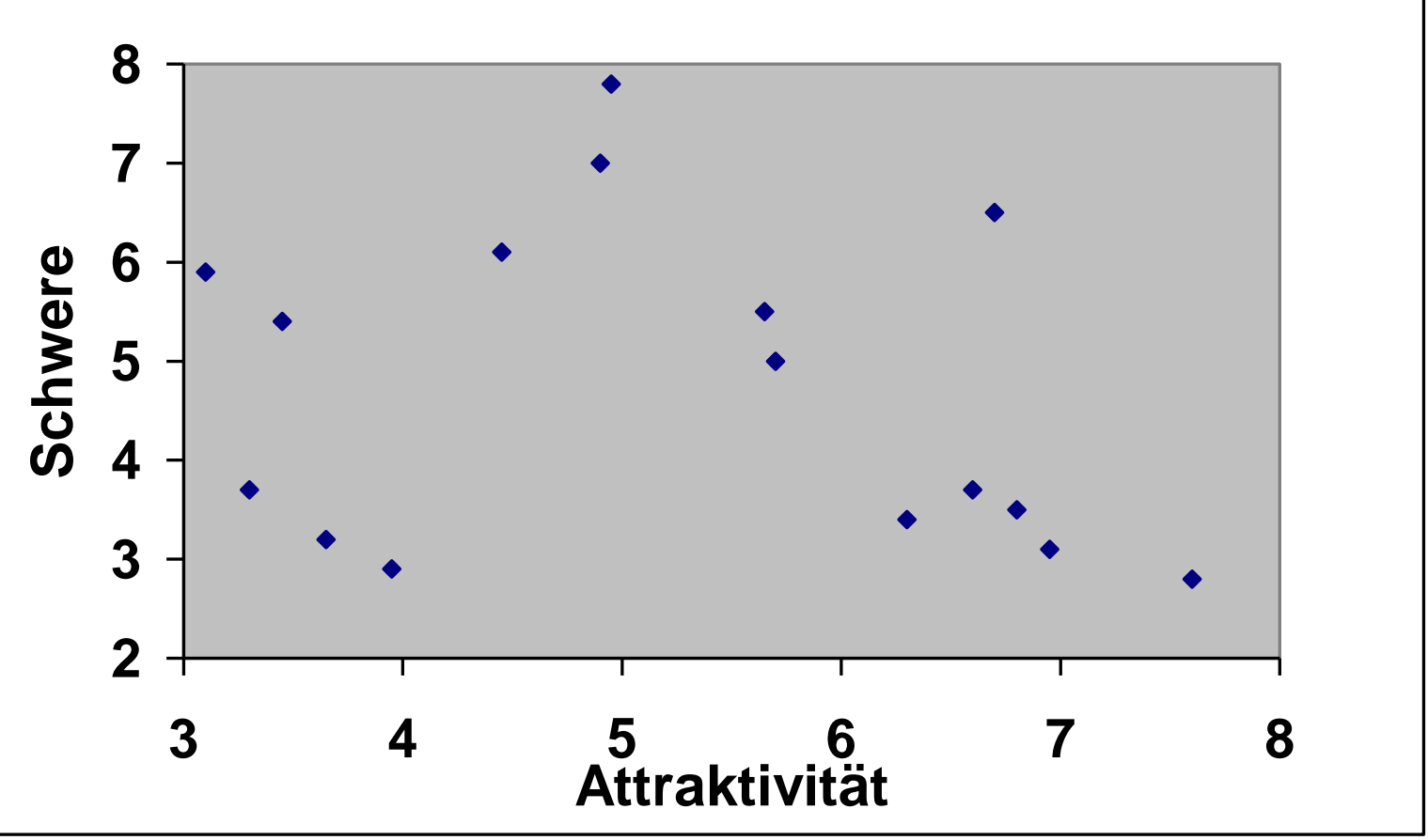

Abb. 10: Beziehung zwischen Attraktivität und Schwere der Hautveränderung bei den Bildvorlagen.

Dass dieser Befund nicht selbstverständlich ist, wird im Kapitel 3.4 bei weiterer Analyse der Beurteilungen noch deutlicher. Als Hinweis auf kognitive und psychodynamische Prozesse, die bei der Bewertung eine Rolle gespielt haben, sei allerdings an dieser Stelle auf die in der Abb. 11 dargestellten Ergebnisse verwiesen. 


\section{Schwere-Beurteilungs-Zeiten bei unterschiedlicher Attraktivität}

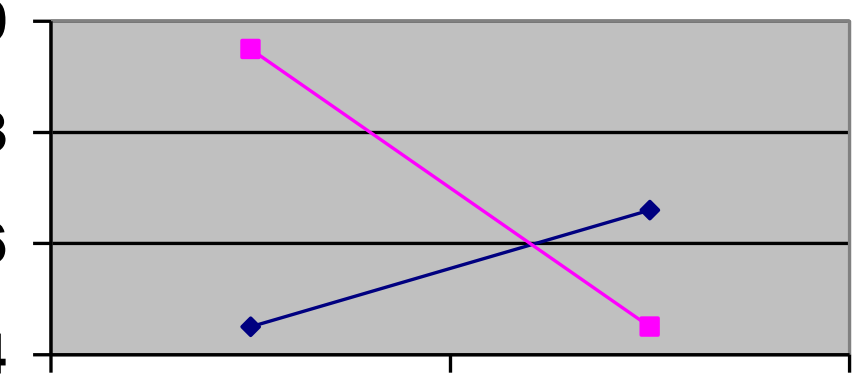

$\rightarrow-$ attraktiv

leicht

schwer

Abb. 11: Unterschiede in den Reaktionszeiten bei Schwere-Beurteilungen der Portraitvorlagen.

(Anmerkung: Wiedergegeben sind hier die Mediane der Reaktionszeiten der jeweils zusammengefassten 4 Gruppen)

$\mathrm{Da}$ das in dieser Studie verwendete RFV-Programm neben der inhaltlichen Urteilsangabe automatisch auch die für jedes einzelne Urteil benötigte Zeit dokumentierte, lässt sich zeigen:

- $\quad$ Reaktionszeit-Mittelwerte zur Abgabe des Schwere-Urteils bei Bildern aus den Gruppen ,attraktiv - leichte Veränderung' und ,unattraktiv - schwere Veränderung' befinden sich in etwa auf einem gleich niedrigen Level,

- $\quad$ während die Schwere-Urteils-Mittelwerte aus den Gruppen ,attraktiv - schwer verändert' und ,unattraktiv - leicht verändert' im Durchschnitt deutlich darüber liegen. In Anlehnung an Konzepte zum Impliziten Assoziationstest (IAT) von Greenwald, McGhee \& Schwartz (1998) lässt sich der Befund wie folgt interpretieren: Die erste Paarung wird als Kongruenz zwischen den jeweiligen Kognitionen Attraktivität und Hautveränderung gewertet, wo "attraktiv“ zu leichten Hautveränderungen und „unattraktiv“ zu schweren Hautveränderungen passt; eine inkongruente Kombination wie ,unattraktiv' und „nur“ ,leichte Hautveränderung' führt demgegenüber sogar zu 
einer mehr als doppelt so langen Urteils-Reaktionszeit; aber auch eine Inkongruenz von ,attraktiv' und ,schwerer Hautveränderung' lässt die Reaktionszeit deutlich ansteigen.

An dieser Stelle werden einige Daten referiert, die veranschaulichen welche Einstellungen die Probandinnen $\mathrm{zu}$ ihrer eigenen Erscheinung und damit wahrscheinlich auch zu dieser Untersuchung mitbrachten. Die Auswertung von Fragen bezüglich ihrer körperlichen Erscheinung ergab ein recht einheitliches Bild: Demnach ist allen Versuchsteilnehmerinnen ihr eigenes Aussehen wichtig gewesen. Jeweils $90 \%$ waren mit ihrem Äußeren eher zufrieden; denken, dass sie über ihr Aussehen Bescheid wissen und mögen es, so wie es ist. Einen abschließenden Blick in den Spiegel, bevor das Haus verlassen wird, tätigen ca. $75 \%$. Wiederum 90\% der Probandinnen halten es für zutreffend, dass es unvermeidlich sei, auf Hautveränderungen zu schauen. 17 der 40 sind oder waren selber schon einmal nach eigenen Angaben von einer Hautkrankheit betroffen.

\subsection{Eignung des RFV-Verfahrens (Hypothese 1)}

In diesem Abschnitt wird der Einsatz der RFV-Technik genauer betrachtet. Dazu werden u.a. persönliche, subjektive Einschätzungen der Probandinnen aus dem Fragenkatalog mit den objektiv gewonnenen Daten verglichen: die persönlichen Eindrücke werden also dem „wirklich“ (unbewusst) gezeigten Blickverhalten gegenübergestellt.

\subsubsection{Verbale Einschätzungen}

Bereits unmittelbar während der Untersuchung zeigte sich, dass die Probandinnen mit der Abarbeitung des Versuchs wenig oder keine Schwierigkeiten hatten. Dennoch gab es im Anschluss an den Versuch persönliche Anmerkungen. Viele Probandinnen fanden die Zwischensequenz "Straßen-Café" „lustig“; einige wenige hatten das Gefühl, die abverlangten Bewertungen wären z.T. „gemein“ gewesen. Selten wurde angemerkt, dass einige Bilder zwei Mal vorkamen. Dies war jedoch nicht allen Teilnehmerinnen bewusst. 
Zum Abschluss wurden alle Teilnehmerinnen per Fragenkatalog bezüglich diverser Aspekte des RFV-Verfahrens befragt (Fragenkatalog s. Anhang). Diese werden im Folgenden referiert.

Um einer "Tendenz zur Mitte“ bei der Beantwortung der Fragen vorzubeugen (Schnell et al. 1999), wurde für die Einschätzungen eine 6er-Skala verwendet. Zur Auswertung ist diese dichotomisiert betrachtet worden; aufgeteilt in die beiden Bereiche 1-3 und 4-6.

Im Resultat hielten 37 der 40 Testpersonen die Betrachtungszeit für ausreichend.

Die Steuerung des Mausfensters wurde unterschiedlich beurteilt. Die Hälfte empfand sie eher irritierend, die andere Hälfte stufte sie als hilfreich ein.

Ähnliches gilt für die Beurteilung der Mausfenstergröße, die $60 \%$ als zu klein befanden und wohl passend dazu den Eindruck hatten, sie wären bei unmittelbarer Betrachtung der Bilder zu anderen Ergebnissen gekommen (80\%).

Das Negativ-Bild als Schattierung kann als durchaus effektiv betrachtet werden, da die Probandinnen ausnahmslos (100\%) der Ansicht waren, die Fotos allein auf Grundlage des Negativs nicht richtig hätten einschätzen zu können.

\subsubsection{Bildhafte Wiedergabe der Effektivität des RFV-Verfahrens}

Auch wenn aus dem zuvor Beschriebenen einige Kritik und Vorbehalte zu entnehmen sind, so zeigt sich in einer ersten deskriptiven und bildhaften Darstellung des Betrachtungsverhaltens der hautkranken Bilder, dass die RFV-Methode geeignet erscheint. 

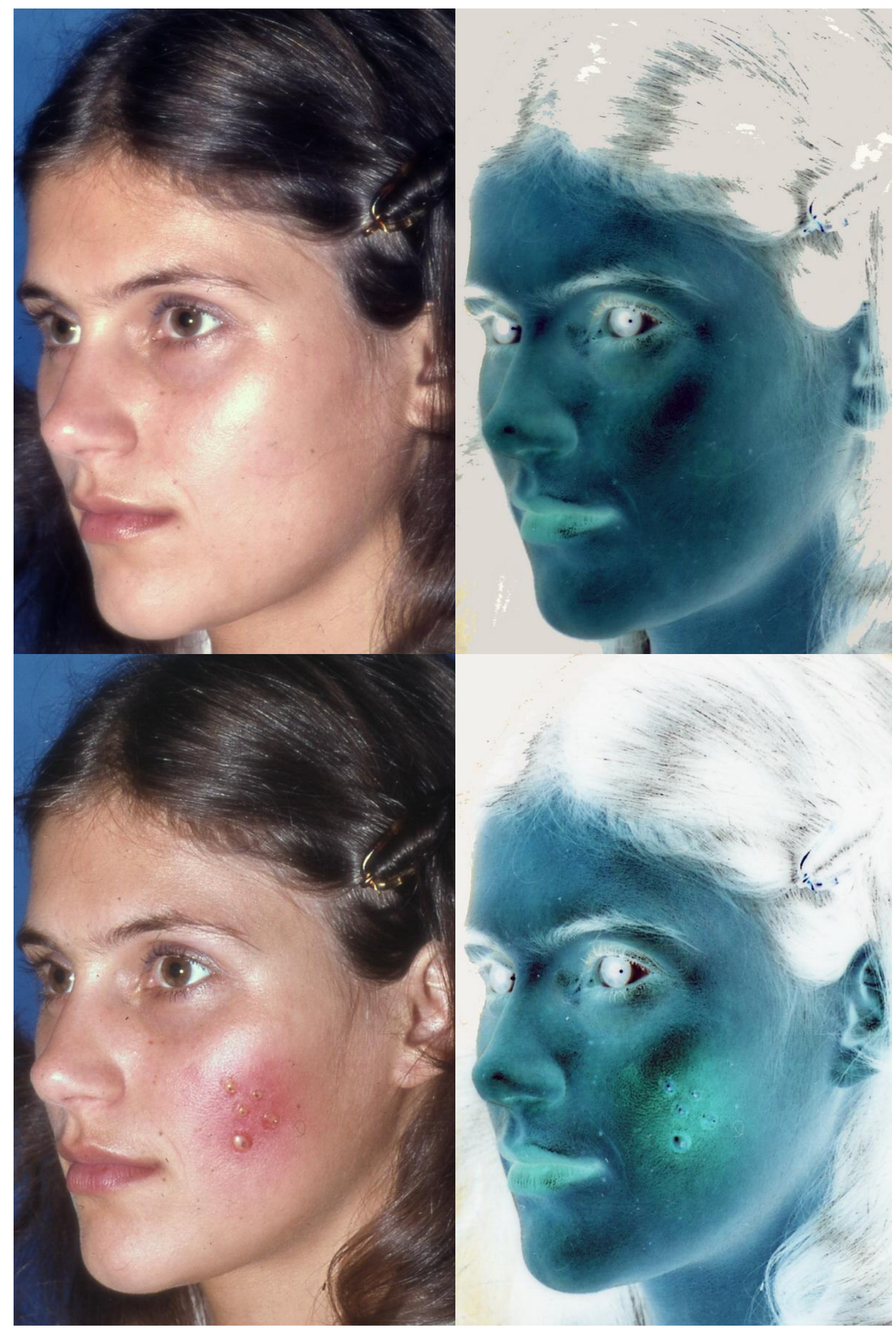


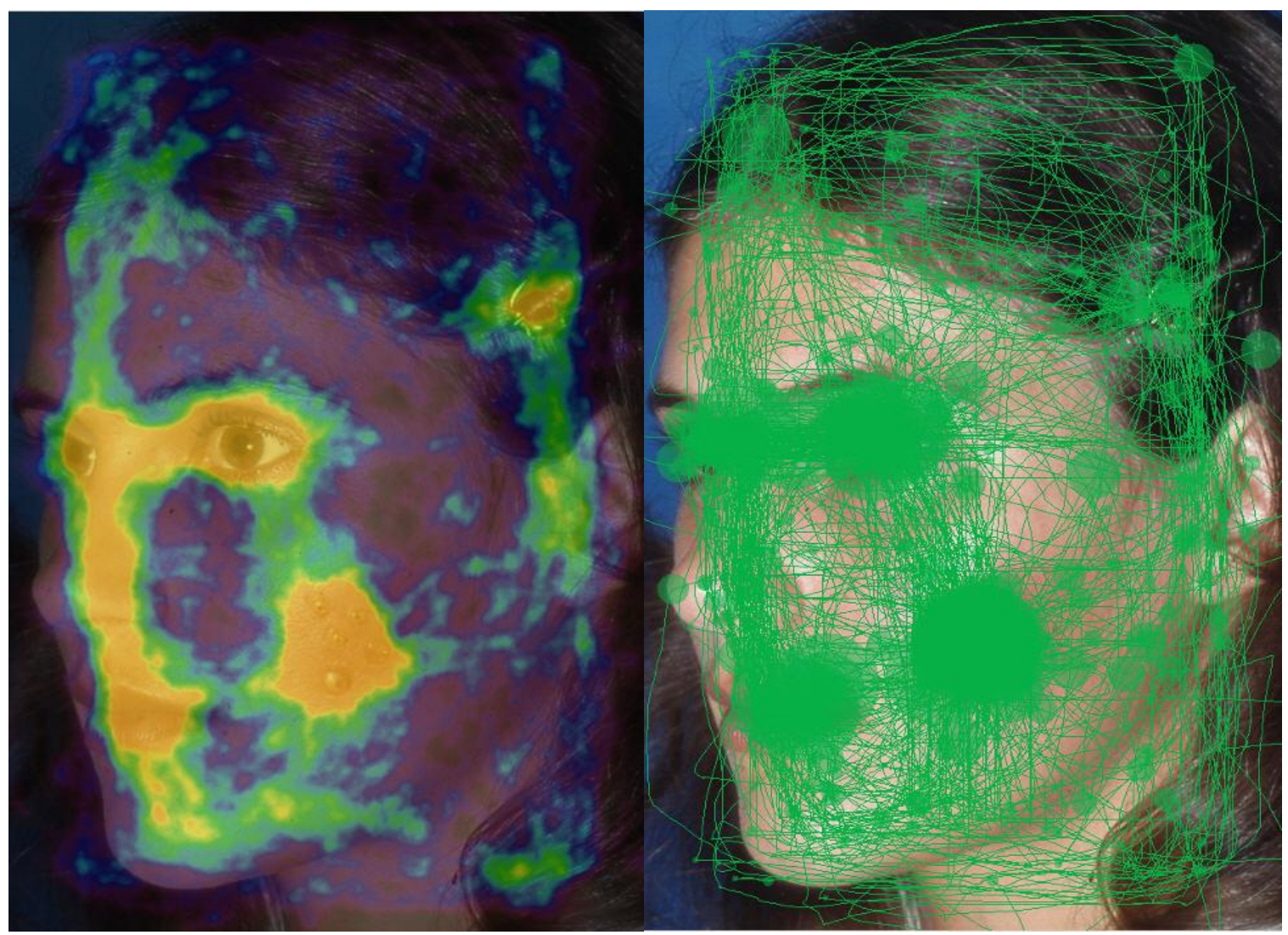

Abb. 12: Exemplarische Darstellung: Bild Nr. 5 im „Original“ und „mit Hautveränderung“ sowie die entsprechende "Negativ-Maskierung“ (oben). Auswertungsbeispiele in der Heatmap- und Scanpath-Darstellung (unten). 

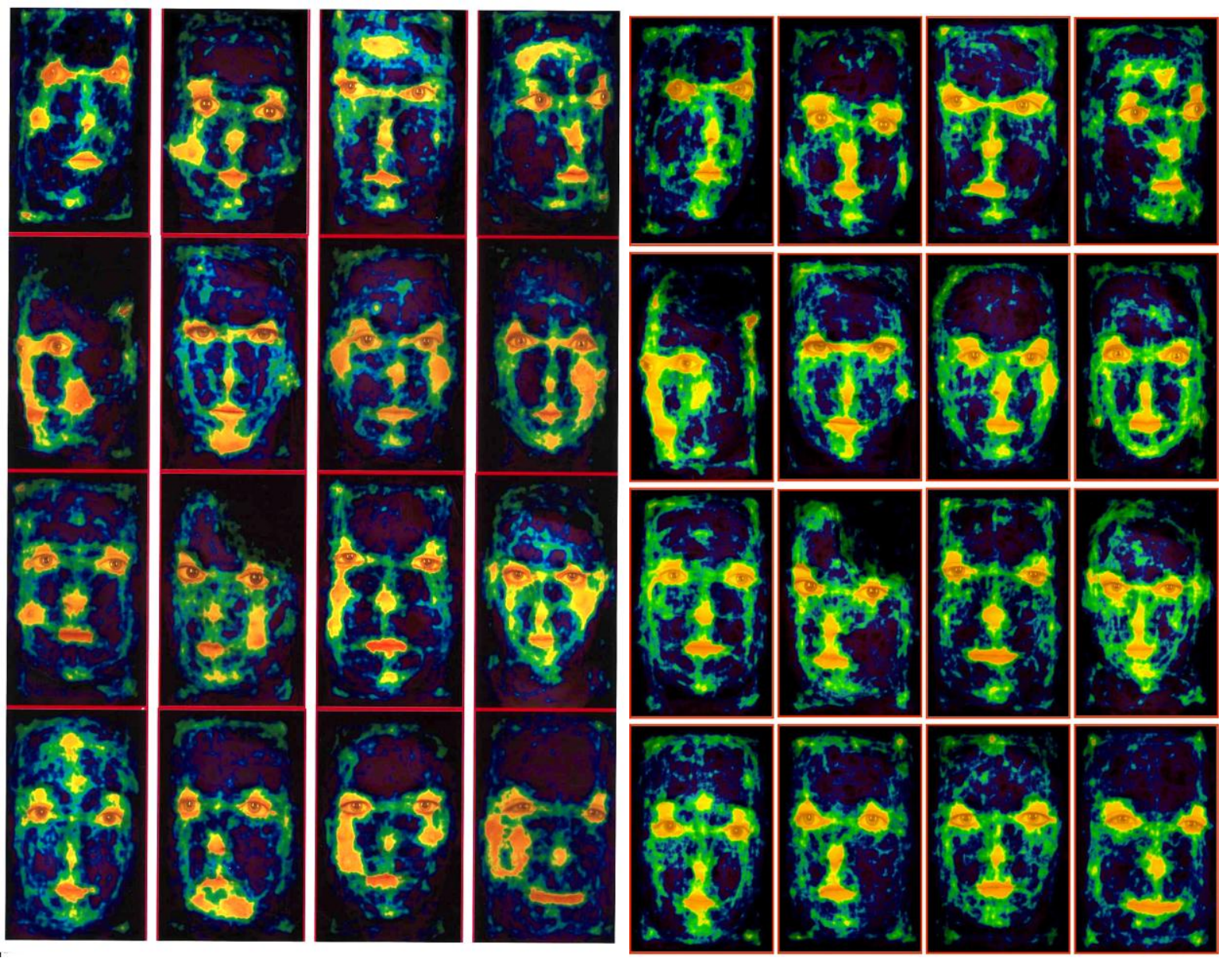

Abb. 13: Heatmap-Darstellung der Betrachtungszeiten von allen Probandinnen. Hier abgebildet sind die Portraits mit Hautveränderungen links und ohne Hautveränderungen rechts.

Wie zuvor bereits in Kapitel (2.5) erläutert, wurden die Blickzeiten u.a. in Form von Heatmaps ausgewertet. Abb. 13 soll einmal die Ergebnisse aller Hg- und Hk-Bilder in dieser Darstellung veranschaulichen. Je länger ein Bereich betrachtet wurde, desto transparenter und leicht rötlich eingefärbt erscheint dieser auf der Heatmap. Im Gegensatz dazu werden alle Bereiche, die weniger betrachtet wurden, zunehmend undurchsichtiger und in dunkleren Blautönen dargestellt. Bei allen Portraits stechen besonders die Bereiche Auge, Nase und Mund hervor. Neben diesen „typisch“ favorisierten Arealen bei der Betrachtung von Gesichtern, fällt jedoch auf, dass in Relation zu anderen Regionen auch die Bereiche mit Hautveränderungen deutlich „transparenter" erscheinen.

Bei den Hg-Bildern gibt es bis auf die Bereiche mit Hautveränderungen weitestgehend gleiche Heatmap-Bilder. 


\subsubsection{Erste numerische Wiedergabe und Auswertung von Blickzeiten}

Erste deskriptiv-statistische Auswertungen - exemplarisch über Mittelwerte - zeigen, dass die "Hitliste" sonstiger Gesichtsbetrachtungsuntersuchungen sich auch hier für hautgesunde Portraits reproduzieren ließ: Die Augen werden am häufigsten angeschaut, gefolgt vom Mund und dann wieder, mit etwas Abstand, Nase und Kinn. Eher weniger, obwohl rein flächenmäßig größer, betrachtet werden „leere Bereiche“ wie Wangen und Stirn.

Die folgende Tabelle zeigt die mittleren Betrachtungszeiten in Sekunden der jeweiligen Areale, zusammengefasst über alle Bilder. Diese wurden für alle 40 Probandinnen bei hautkrankem $(\mathrm{Hk})$ und hautgesundem $(\mathrm{Hg})$ Bild verglichen.

\begin{tabular}{|c|c|c|c|c|c|c|}
\hline & \multicolumn{3}{|c|}{$\begin{array}{c}\text { Blickzeiten Hk-Bilder } \\
\text { (in Sekunden) }\end{array}$} & \multicolumn{3}{c|}{$\begin{array}{c}\text { Blickzeiten Hg-Bilder } \\
\text { (in Sekunden) }\end{array}$} \\
\hline Areale & Mittelwert & Streuung & Median & Mittelwert & Streuung & Median \\
\hline Stigma & 4,54 & 2,89 & 3,82 & 1,65 & 1,51 & 1,38 \\
\hline Auge links & 5,01 & 2,41 & 4,60 & 5,42 & 2,71 & 5,01 \\
\hline Auge rechts & 4,44 & 2,12 & 4,20 & 4,87 & 2,28 & 4,49 \\
\hline Mund & 2,99 & 1,74 & 2,68 & 3,50 & 1,82 & 3,18 \\
\hline Nase & 2,18 & 1,35 & 2,02 & 2,69 & 1,69 & 2,47 \\
\hline Haare & 2,42 & 1,65 & 2,09 & 2,79 & 1,92 & 2,54 \\
\hline Stirn & 1,60 & 2,00 & 1,27 & 1,27 & 1,45 & 0,94 \\
\hline Kinn & 2,84 & 2,00 & 2,53 & 2,72 & 1,63 & 2,56 \\
\hline Wange links & 2,44 & 2,52 & 1,68 & 1,47 & 1,36 & 1,22 \\
\hline Wange rechts & 2,30 & 2,66 & 1,51 & 1,29 & 1,46 & 1,02 \\
\hline
\end{tabular}

Tab. 1: Blickzeitwerte für verschiedene Areale auf den Portraits mit und ohne Hautveränderungen. (Anmerkung: Areale mit/ohne Stigma sind „kursiv“; Gesamtbetrachtungszeit: 30 Sekunden)

Bei hautkranken Portraits bekommt das Stigma-Areal die zeitliche Wertigkeit von Augen. Im Vergleich von Hk- zu Hg-Bildern liegen die Stigma-Areal-Fixationszeiten außerordentlich deutlich auseinander (ein bis zwei Standardabweichungen). Damit werden Befunde aus früheren Untersuchungen (beispielsweise Brüninghaus 1980, 
Kahle 2007) nicht nur reproduziert, sondern auch durch die zugrunde liegende große Probandinnen-Zahl $(\mathrm{n}=40)$ abgesichert.

Anhand einiger ausgewählter Bilder (in denen die abgebildeten Portraitpersonen en face und mit direktem Blick in Richtung Betrachter zu sehen sind) lässt sich darüber hinaus ein weiteres Phänomen reproduzieren: der left gaze bias.

Bei Vorliegen eines left gaze bias, also einer erhöhten Aufmerksamkeit für das eigene linke Gesichtsfeld bzw. die rechte Gesichtshälfte des Portraits, muss eine signifikante Differenz in der Betrachtungszeit zwischen der rechten und linken Gesichtshälfte bestehen (Guo et al. 2012; Guo et al. 2009; Ricciardelli et al. 2002).

Zur Bestimmung des left gaze bias wurden die Fixationszeiten (FZ) für das linke und rechte Auge aus Sicht der Betrachterinnen miteinander verglichen. In der Tabelle sind die mittleren Differenzen in Millisekunden wiedergegeben. Bestimmte Bilder mussten ausgeschlossen werden, da qualitative bzw. quantitative Unterschiede zwischen den Augen bestanden: verschiedene Blickrichtungen nach links oder rechts sowie unterschiedliche Größen der Augenfelder.

\begin{tabular}{|c|l|c|c|c|c|c|c|}
\hline & & \multicolumn{3}{|c|}{ Hg-Portrait } & \multicolumn{3}{c|}{ Hk-Portrait } \\
\hline $\begin{array}{c}\text { Bild- } \\
\text { Nr. }\end{array}$ & & Differenz & t-Wert & $\mathrm{p}$-Wert & Differenz & t-Wert & $\mathrm{p}$-Wert \\
\hline 2 & & 2216,8 & 4,00 & 0,0003 & 2500,6 & 4,61 & $<0,0001$ \\
\hline 6 & & 315,5 & 0,85 & 0,40 & 1604,9 & 3,15 & 0,003 \\
\hline 8 & & 415,9 & 1,12 & 0,27 & 337,8 & 1,00 & 0,32 \\
\hline 10 & & 393,3 & 0,84 & 0,41 & 279,2 & 0,53 & 0,60 \\
\hline 11 & & 1028,8 & 1,24 & 0,22 & 676,6 & 1,93 & 0,62 \\
\hline 12 & & 381,7 & 1,03 & 0,31 & 308,4 & 1,11 & 0,28 \\
\hline 13 & & 914,2 & 2,20 & 0,03 & 1758,3 & 4,21 & 0,0001 \\
\hline 15 & & 8,9 & 0,01 & 0,99 & 388,1 & 0,73 & 0,47 \\
\hline & & & & & & \\
\hline & Zusammen & & & & & & \\
\hline & \multirow{2}{*}{$\begin{array}{l}\text { fassung } \\
\text { Bilder) }\end{array}$} & $\mathbf{7 0 9 , 4}$ & 3,71 & 0,0002 & $\mathbf{9 8 1 , 7}$ & 6,03 & $<0,0001$ \\
\cline { 2 - 8 } & $\mathrm{s}=3421,2$ & & & $\mathrm{~s}=2914,4$ & & \\
\cline { 2 - 8 } & $\mathrm{df}=319$ & & & $\mathrm{df}=319$ & & \\
\hline
\end{tabular}

Tab. 2: Zeitdifferenz bei der Betrachtung des linken gegenüber dem rechten Auge beim gesunden und kranken Portrait - einzeln und summarisch.

(Anmerkung: Differenz = FZ (linkes Auge) - FZ (rechtes Auge); Zeitwerte in Millisekunden) 
Zwar fielen die Unterschiede im Hinblick auf den left gaze bias bei den einzelnen Bildern unterschiedlich stark aus - aber in der Tendenz sind sie bei allen Portraits gegeben. Die zusammenfassende Analyse zum left gaze bias von 8 geeigneten

Bildern bei 40 Probandinnen zeigt:

- $\quad$ bei den gesunden Bildern $(\mathrm{Hg})$ eine hoch signifikante mittlere Differenz von etwa 0,7 Sekunden

- $\quad$ bei den kranken Bildern (Hk) eine hoch signifikante mittlere Differenz von etwa 1 Sekunde

Zusammenfassend lässt sich festhalten: Trotz einiger von den Teilnehmerinnen vorgebrachter Vorbehalte gegen Zwänge und Restriktionen der RFV-Technik nach den Testdurchläufen ist (u.a. anhand der Messwerte zu unterschiedlichen Phänomenen) das Verfahren in der gewählten Form (Negativ-Positiv-Darbietung) objektiv geeignet, Betrachtungsverhalten gegenüber Portraits mit und ohne Hautveränderung zu erfassen.

\subsection{Blickverhalten und das Schema-Konzept (Hypothese 2)}

Als theoretische Grundlage diente das "Schema-Konzept" (Cash 1989; Henss 1998; Murray et al. 2003), wonach mit Hilfe kognitiver Strukturen und des Sinnesapparates in dieser Studie der Augen ein bestehendes, bekanntes Schema mit aktuellen Reizen verglichen und gegebenenfalls angepasst wird. Die Anpassung kann in Form von Assimilation oder Akkomodation erfolgen. Bekannte Reize oder solche, die nur kleine Abweichungen zu bestehenden Schemata aufweisen, bedürfen lediglich assimilatorischer Verarbeitung, während schemainkongruente Reize eine Akkomodation des Schemas notwendig machen (Garcia-Marques et al. 2004; Peskin und Newell 2004). Die Annahme lautete demnach: Je leichter Reize in ein „aktiviertes Schema" integrierbar sind (z.B. hautgesunde Bilder), desto schneller werden sie verarbeitet und obendrein später als attraktiver bewertet. Im Umkehrschluss wird ein mit dem schemainkongruenter Reiz langsamer verarbeitet.

Zusammenfassend betrachtet werden also bekannte Reize schneller verarbeitet, während unbekannte und unerwartete Reize mehr Aufmerksamkeit für die Verarbeitung benötigen, zeitlich gesehen also länger betrachtet werden (Barton et al. 2006; Wohlrab et al. 2007). 


\subsubsection{Aufmerksamkeitsveränderungen im Blickverhalten aufgrund von Hautveränderungen}

Die erste Annahme bezog sich auf Veränderungen im Blickverhalten bei zwei weitestgehend identischen Experimentalbildern, die sich nur dahingehend unterscheiden, dass die abgebildete Person auf dem einen hautgesund $(\mathrm{Hg})$ und auf dem anderen mit umschriebenen hautkranken $(\mathrm{Hk})$ Veränderungen präsentiert wird. Als Indikator der Aufmerksamkeit diente hierzu die registrierte Verweildauer des Fokusfensters im Stigma-Areal. Es müsste sich also ein signifikanter Unterschied der Betrachtungszeiten in den Bereichen ergeben, welche im Gegensatz zum sonst identischen hautgesunden Foto eine Hautveränderung aufweisen. Ein diesbezüglich zusammenfassender Befund ist bereits in Abschnitt 3.2.3 referiert worden, so dass in der hier folgenden Tabelle die Daten differenziert nach den einzelnen Bildern wiedergegeben werden. 


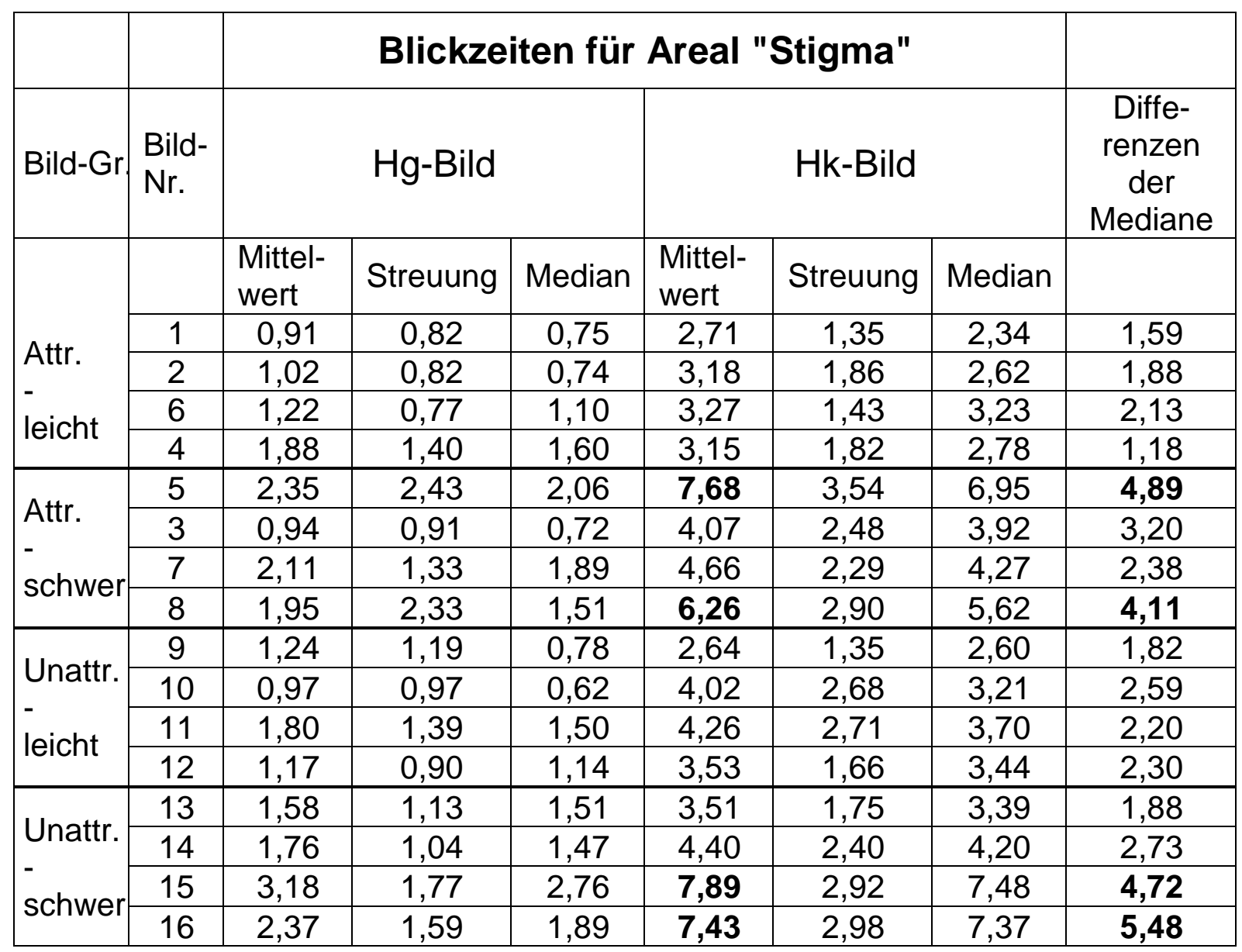

Tab. 3: Einzelbildanalyse der Blickzeiten für das Areal "Stigma".

(Anmerkung: Blickzeiten in Sekunden, Probandinnen-Zahl ( $n=40$ ); Bild 3 und 6 tauschen die Gruppenzugehörigkeit auf Grund stichprobenbedingter veränderter Schwere-Einschätzung)

Wie sich aus der Tabelle entnehmen lässt, waren die Betrachtungszeiten der "Stigma-Areale" bei den hautkranken Experimentalfotos ( $\mathrm{Hk}$ ) im Mittel ausnahmslos höher, als bei den entsprechenden hautgesunden $(\mathrm{Hg})$ Pendants. Folglich betrachteten also im Durchschnitt alle Versuchsteilnehmerinnen die veränderten Stellen bei allen hautkranken Bildern länger als bei den hautgesunden Vorlagen.

Diese Betrachtungsdifferenz zwischen $\mathrm{Hg}$ - und Hk-Bildern (bezogen auf die Mediane) bewegte sich in einem Rahmen von mindestens 1,18 sec bei Bild 4 und maximal 5,48 sec bei Experimentalbild Nr. 16. Für die Streuungen wurde ein Bereich zwischen 0,77 sec bei Bild Nr. $6(\mathrm{Hg})$ und 3,54 sec bei Bild Nr. 5 (Hk) ermittelt. Hervorzuheben sind darüber hinaus die besonders hohen bzw. langen Betrachtungszeiten der Stigma-Areale von den Hk-Bildern 5, 8, 15 und 16 (Bild 5 und 8 in der Gruppe „eher attraktiv“, Bild 15 und 16 hingegen gehörten zur Gruppe „eher 
unattraktiv"). Die Hautveränderungen sind im Vorfeld als "schwer verändert" eingestuft worden. Das Blickverhalten änderte sich also nicht nur deutlich im allgemeinen Vergleich, sondern auch in verschiedenen Untergruppen: die FZMittelwerte der als "schwer verändert" eingestuften Bilder liegen deutlich und sogar erheblich über den vergleichsweise "leicht veränderten“ derselben Gruppe. Es ist dabei allerdings auch zu betonen, dass dies scheinbar unabhängig von der Attraktivitätseinschätzung geschieht, sodass jeweils die Gruppen „attraktiv-leicht verändert" gegenüber „unattraktiv-leicht verändert" bzw. „attraktiv-schwer verändert" gegenüber „unattraktiv-schwer verändert“ vergleichbare Werte aufweisen.

Im Ergebnis kann das Blickverhalten als signifikant verändert bezeichnet werden. Dabei nimmt die Betrachtungszeit nicht nur aufgrund allgemeiner Veränderung der Haut, sondern auch in Abhängigkeit des Schweregrades der Veränderung zu - und das insgesamt unabhängig vom Faktor Attraktivität.

\subsubsection{Aufmerksamkeitsveränderungen als Folge der Wahrnehmungs-Verarbeitung}

Im Rahmen des Schema-Konzepts ist weiterhin zu erwarten, dass in der ersten Hälfte der Gesamt-Betrachtungszeit die Fixationszeiten für Stigma-Areale höher sind, als in der zweiten Hälfte, in der schon eine gewisse Verarbeitung des "Neuen“ stattgefunden haben müsste. Wie in der folgenden Tabelle (Tab. 4) deutlich wird, zeigt sich ein derartiger, hochsignifikanter Aufmerksamkeits-Effekt bezogen auf die Hk-Bilder, welcher auch erhalten bleibt, wenn die Werte nach Präsentationsfolge aufspaltet werden.

Berechnet man zur Kontrolle bei den $\mathrm{Hg}$-Bildern für das identische Areal ohne jegliche Hautveränderung die Fixationszeiten, so weisen diese keinen bedeutsamen Zeiteffekt auf. 


\begin{tabular}{|c|c|c|c|c|c|c|}
\hline & \multicolumn{6}{|c|}{ Stigma-Areal-Fixationszeiten } \\
\hline & \multicolumn{3}{|c|}{ Hk-Bild } & \multicolumn{3}{|c|}{ Hg-Bild (Kontrolle) } \\
\hline Betrachtung & Mittelwert & Streuung & Median & Mittelwert & Streuung & Median \\
\hline 1. Hälfte & 2,60 & 1,70 & 2,21 & 0,86 & 0,84 & 0,64 \\
\hline 2. Hälfte & 1,94 & 1,83 & 1,49 & 0,79 & 1,09 & 0,53 \\
\hline $\begin{array}{c}\text { Paar- } \\
\text { Differenz }\end{array}$ & 0,66 & & 0,72 & 0,07 & & 0,11 \\
\hline tpaar & 8,72 & & & 1,43 & & \\
\hline$d f$ & 639 & & & 639 & & \\
\hline$p$ & $<0,0001$ & & & 0,15 & & \\
\hline
\end{tabular}

Tab. 4: Differenz der Stigma-Areal-Fixationszeiten zwischen 1. und 2. Hälfte der Gesamtbetrachtungszeit.

(Anmerkung: Zeit in Sek.)

Des Weiteren lässt sich im Rahmen des Schema-Konzepts erwarten, dass es zu einem deutlichen zeitlichen Positionseffekt kommt, in Abhängigkeit der Reihenfolge in der die Experimentalfotos gezeigt werden - also ob zuerst das hautkranke und dann das hautgesunde Bild gesehen wird oder umgekehrt. Für Hk-Bilder die an zweiter Stelle gezeigt werden, ist mit einem Anstieg der Betrachtungszeiten zu rechnen. Dies lässt sich als eine Art "doppelter Neuigkeitseffekt" beschreiben: Die hautveränderten Areale sind nämlich dann an zweiter Stelle für den Betrachter nicht nur grundsätzlich „ungewöhnlich und neu“, sondern auch „relativ-neu“ gegenüber den schon zuvor gesehenen und damit gar nicht mehr so neuen anderen Arealen des an erster Stelle präsentierten Hg-Bildes (wie Augen, Nase, Mund etc.). Wie in der Tabelle (Tab. 5) dokumentiert wird, fallen die Fixationswerte für hautkranke Areale, so sie an zweiter Stelle präsentiert werden, in der Tat höher aus als bei der Präsentation an erster Stelle. Dies gilt sowohl für die Gesamtwerte, als auch für die Teil-Werte einer einzelnen Bildbetrachtung aus der ersten (Sekunde 1-15) und zweiten Hälfte (Sekunde 15-30). Die Stigma-Fixationszeit nimmt allerdings in der zweiten Hälfte, aufgrund der Gewöhnung an die Reiz-Neuartigkeit, wie erwartet ab. 


\begin{tabular}{|c|c|c|c|c|c|}
\hline & & $\begin{array}{l}\text { la-Areal-Fi } \\
\text { (alle Hk-E }\end{array}$ & $\begin{array}{l}\text { tionszeiten } \\
\text { ler) }\end{array}$ & & \\
\hline $\begin{array}{l}\text { Präsentations- } \\
\text { Folge: }\end{array}$ & Gesamt & $\begin{array}{c}\text { 1. Hälfte } \\
\text { (Sek. 1-15) }\end{array}$ & $\begin{array}{c}\text { 2. Hälfte } \\
\text { (Sek. 15-30) }\end{array}$ & \multicolumn{2}{|c|}{$\begin{array}{c}\text { Nebenbefund: } \\
\text { Zeitliche Paar- } \\
\text { Differenz }\end{array}$} \\
\hline \multirow[t]{2}{*}{ an 1. Stelle } & \multirow[t]{2}{*}{4,07} & \multirow[t]{2}{*}{2,31} & \multirow[t]{2}{*}{1,77} & \multirow[t]{2}{*}{0,54} & $\begin{aligned} & t_{\text {paar }}=5,30 \\
& \mathrm{df}=319\end{aligned}$ \\
\hline & & & & & $p<0,0001$ \\
\hline \multirow{3}{*}{ an 2. Stelle } & \multirow{3}{*}{5,01} & \multirow{3}{*}{2,90} & \multirow{3}{*}{2,11} & \multirow{3}{*}{0,78} & tpaar $=6,30$ \\
\hline & & & & & $d f=319$ \\
\hline & & & & & $p<0,0001$ \\
\hline $\mathrm{t}$ & $-4,15$ & $-4,47$ & $-2,39$ & \multirow{3}{*}{\multicolumn{2}{|c|}{$\begin{array}{l}\text { bei ungleichen Varianzen } \\
\text { Freiheitsgrade df } 638 \\
\text { (Satterthwaite-Korrektur) }\end{array}$}} \\
\hline $\mathrm{df}$ & 588,41 & 585,89 & 606,16 & & \\
\hline$p$ & $<0,0001$ & $<0,0001$ & 0,0172 & & \\
\hline
\end{tabular}

Tab. 5: Stigma-Areal-Fixationszeiten differenziert nach Präsentations-Folge. (Anmerkung: Als Nebenbefund sind hier noch einmal Vergleiche zwischen den beiden Zeithälften der Betrachtung aufgegliedert. Zusätzlich wird nach PräsentationsFolgen differenziert - vgl. Tab. 4)

Schließlich ist ein Schema-Priming-Effekt für das Stigma-Areal zu prüfen: Wenn HgBilder an zweiter Stelle gezeigt werden, müsste es dem Effekt zur Folge zu einer signifikant höheren Betrachtungszeit im vorher veränderten, nun aber hautgesunden Areal kommen im Vergleich zu Hg-Bildern, die an erster Stelle und damit ohne vorausgehendes Hk-Bild zu sehen waren (Hünecke 2009: „Franzky-Effekt“). 


\section{Stigma-Areal-Fixationszeiten \\ (alle Hg-Bilder)}

\begin{tabular}{|c|c|c|c|}
\hline $\begin{array}{l}\text { Präsentations- } \\
\text { Folge: }\end{array}$ & Gesamt & 1. Hälfte & 2. Hälfte \\
\hline an 1. Stelle & 1,55 & 0,80 & 0,75 \\
\hline an 2. Stelle & 1,76 & 0,93 & 0,83 \\
\hline $\mathrm{t}$ & $-1,8$ & $-2,01$ & $-0,94$ \\
\hline $\mathrm{df}$ & 581,57 & 638 & 554,27 \\
\hline $\mathrm{p}$ & 0,07 & 0,05 & 0,35 \\
\hline
\end{tabular}

Tab. 6: Fixationszeiten der Stigma-Areale auf den Hg-Portraits, differenziert nach Präsentations-Folge sowie Betrachtungshälfte.

(Anmerkung: bei ungleichen Varianzen Freiheitsgrade $\mathrm{df}<638$ [SatterthwaiteKorrektur, SAS „TTEST Procedure“])

Für alle Bilder ist dieser Effekt über die gesamte Betrachtungszeit nur tendenziell zu sichern ( $p>0,05$ aber $p<0,10)$. Zieht man jedoch ausschließlich die entsprechenden Zeitwerte aus der ersten Präsentationshälfte heran, so ist der Effekt als signifikant nachzuweisen - um dann allerdings auf den Messergebnissen der zweiten Hälfte als nicht-existent gewertet zu werden.

Differenziert man obige Präsentations-Folge- und Priming-Effekte, indem man die Unterschiede zwischen den Blickzeiten für das Areal "Stigma" pro Bild einzeln analysiert, so zeigt sich:

- Mit Ausnahme von Bildern 7 und 12 ließ sich einzeln bei den Hk-Bildern eine bedeutsam höhere Betrachtungszeit an zweiter Stelle (Mittelwert-2) nachweisen gegenüber den Fixationszeiten bei Hk-Bildern, die an erster Stelle präsentiert wurden (Mittelwert-1).

- $\quad$ Ein vergleichbarer systematischer Positionseffekt ist bei den $\mathrm{Hg}$-Bildern, mit Ausnahme der Bilder 5, 8 und 15, nicht zu erkennen. Diese Bilder erhielten zusammen mit dem Bild 16 die höchsten Bewertungen in Bezug auf das Attribut „Auffälligkeit".

- $\quad$ Die als auffällig bewertete Veränderung des Hk-Bildes wird bei Präsentation an zweiter Stelle im Areal des Hg-Bildes quasi „aufgesucht“ (erhöhte Aufmerksamkeit). Das zuvor aktuell angeeignete Schema ,schwer-hautkrank-verändert' ist quasi unvereinbar mit dem nun hautgesunden Zustand und erzwingt die erhöhte 
Aufmerksamkeit, obwohl rein physikalisch eigentlich „nichts“ Verändertes wahrzunehmen ist.

\begin{tabular}{|l|c|c|c|c|}
\hline & \multicolumn{2}{|c|}{ Präsentations-Folge-Effekt } & \multicolumn{2}{c|}{ Priming-Effekt } \\
\hline $\begin{array}{l}\text { Bild- } \\
\text { Gruppe }\end{array}$ & \multicolumn{2}{|c|}{ Hk-Bild } & \multicolumn{2}{c|}{ Hg-Bild } \\
\hline $\begin{array}{l}\text { Bild- } \\
\text { Nummer }\end{array}$ & Mittelwert-1 & Mittelwert-2 & Mittelwert-1 & Mittelwert-2 \\
\hline 1 & 2,28 & $\mathbf{3 , 1 3}$ & 0,77 & 1,05 \\
\hline 2 & 2,63 & $\mathbf{3 , 7 4}$ & 1,13 & 0,91 \\
\hline 6 & 2,92 & $\mathbf{3 , 6 1}$ & 1,16 & 1,28 \\
\hline 4 & 2,97 & $\mathbf{3 , 3 3}$ & 1,88 & 1,89 \\
\hline 5 & 6,70 & $\mathbf{8 , 6 7}$ & 1,68 & $\mathbf{3 , 0 2}$ \\
\hline 3 & 3,36 & $\mathbf{4 , 7 7}$ & 1,00 & 0,87 \\
\hline 7 & 4,64 & 4,67 & 2,16 & 2,07 \\
\hline 8 & 5,41 & $\mathbf{7 , 1 0}$ & 1,39 & $\mathbf{2 , 5 0}$ \\
\hline 9 & 2,31 & $\mathbf{3 , 0 0}$ & 1,08 & 1,40 \\
\hline 10 & 3,40 & $\mathbf{4 , 6 5}$ & 0,85 & 1,08 \\
\hline 11 & 3,33 & $\mathbf{5 , 1 8}$ & 1,92 & 1,69 \\
\hline 12 & 3,90 & 3,17 & 1,06 & 1,29 \\
\hline 13 & 2,84 & $\mathbf{4 , 1 8}$ & 1,73 & 1,43 \\
\hline 14 & 3,73 & $\mathbf{5 , 0 7}$ & 1,77 & 1,74 \\
\hline 15 & 7,41 & $\mathbf{8 , 3 7}$ & 2,80 & $\mathbf{3}, \mathbf{5 6}$ \\
\hline 16 & 7,32 & $\mathbf{7 , 5 5}$ & 2,38 & 2,37 \\
\hline
\end{tabular}

Tab. 7: Bildspezifische Zeit-Effekte.

(Anmerkung: Bild 3 und 6 tauschen die Gruppenzugehörigkeit auf Grund stichprobenbedingter veränderter Schwere-Einschätzung)

\subsection{Beziehung Stigma-Fixationszeit und Bewertung (Hypothesen 3a, 3b, 3c)}

Die nächsten zu prüfenden Hypothesen beziehen sich auf den Zusammenhang zwischen Fixationszeiten für Hautveränderungen und Bewertungen. Es wird auf der einen Seite eine eindeutige Korrelation zwischen der Länge der StigmaFixationszeiten und der Ungewöhnlichkeits- bzw. Auffälligkeitseinstufung erwartet. Auf der anderen Seite wird ein nur sehr geringer bzw. gar kein Zusammenhang zwischen der Aufmerksamkeitssteigerung bzw. Blickzeitverlängerung und der Attraktivitäts- bzw. Angenehmheits-Bewertung angenommen. 


\subsubsection{Eingehende Überprüfung der Bewertungen: Präsentationsfolge-Effekt und Dimensions-Unabhängigkeit}

Bevor die eigentlichen Zusammenhangs-Berechnungen erfolgen können, sind einige Vorarbeiten von Nöten. Es lassen sich nämlich, wie für die Fixationszeiten (s. Tab. 7), auch für die Bewertungen - und hier besonders für die Attraktivitätseinstufungen - so genannte „Präsentations-Positions-Effekte“ aufzeigen.

Im Folgenden wird zunächst die Attraktivitäts-Bewertung betrachtet.

Die Tabelle 8 listet für jedes Experimentalfoto, $\mathrm{Hg}$ und $\mathrm{Hk}$, die dazugehörigen Mittelwerte des Attraktivitätsratings je nach seiner Präsentationsposition auf. Insgesamt fallen die Attraktivitäts-Bewertungen für die hautgesunden Bilder positiver aus als für die hautkranken. 


\begin{tabular}{|c|c|c|c|c|c|c|}
\hline \multirow{3}{*}{$\begin{array}{c}\begin{array}{c}\text { Bild- } \\
\text { Gruppe }\end{array} \\
\text { Bild- } \\
\text { Nummer }\end{array}$} & \multicolumn{3}{|c|}{ Hk-Bild } & \multicolumn{3}{|c|}{ Hg-Bild } \\
\hline & 1. Stelle & & 2. Stelle & 1. Stelle & & 2. Stelle \\
\hline & Mittelwert-1 & & Mittelwert-2 & Mittelwert-1 & & Mittelwert-2 \\
\hline 1 & 7,10 & $<$ & 7,70 & 7,6 & $<$ & 8,05 \\
\hline 2 & 6,85 & $<$ & 7,20 & 6,95 & $<$ & 7,65 \\
\hline 6 & 6,70 & $>$ & 6,20 & 6,8 & $<$ & 7,65 \\
\hline 4 & 5,70 & $<$ & 6,70 & 6,6 & $>$ & 5,7 \\
\hline 5 & 5,25 & $>$ & 4,90 & 6,7 & $<$ & 7,4 \\
\hline 3 & 4,70 & $<$ & 5,25 & 5,7 & $<$ & 6,6 \\
\hline 7 & 5,00 & $=$ & 4,95 & 5,65 & $<$ & 6,05 \\
\hline 8 & 3,40 & $<$ & 4,25 & 4,9 & $=$ & 4,95 \\
\hline 9 & 4,05 & $<$ & 4,65 & 3,95 & $<$ & 4,25 \\
\hline 10 & 3,65 & $<$ & 4,60 & 3,65 & $<$ & 4,5 \\
\hline 11 & 3,60 & $=$ & 3,50 & 3,3 & $<$ & 3,9 \\
\hline 12 & 4,65 & $<$ & 5,60 & 6,3 & $>$ & 5,3 \\
\hline 13 & 3,20 & $<$ & 3,55 & 3,45 & $<$ & 4,1 \\
\hline 14 & 2,70 & $<$ & 2,90 & 3,1 & $<$ & 4,05 \\
\hline 15 & 3,50 & $<$ & 3,85 & 4,95 & $<$ & 5,75 \\
\hline 16 & 3,85 & $=$ & 3,80 & 4,45 & $>$ & 3,75 \\
\hline $\begin{array}{c}\text { Alle } \\
\text { Bilder }\end{array}$ & 4,62 & $<$ & 5,00 & 5,25 & $<$ & 5,60 \\
\hline \multirow[t]{2}{*}{$s$} & 2,08 & & 2,06 & 2,08 & & 2,09 \\
\hline & $\mathrm{df}=638$ & \multicolumn{2}{|c|}{$t=-2,18$} & $d f=638$ & $=-2,12$ & $p=0,03$ \\
\hline
\end{tabular}

Tab. 8: Auflistung der Mittelwerte der Attraktivitätsbewertung ( $n=40)$, differenziert nach Präsentationsfolge.

(Anmerkung: Bild 3 und 6 tauschen die Gruppenzugehörigkeit auf Grund stichprobenbedingter veränderter Schwere-Einschätzung)

\section{Bezüglich der Präsentationsfolge}

- fallen bei Hk-Bildern die absoluten Attraktivitätsurteile in 11 Fällen besser aus, 3mal gleich und 2 mal schlechter;

- fallen bei Hg-Bildern die absoluten Attraktivitätsurteile in 12 Fällen besser aus, 1 mal gleich und 3 mal schlechter.

D.h. beim ersten Sehen wird kritischer und/oder beim zweiten Sehen milder geurteilt. Zusammengefasst über alle Bilder (und Probandinnen) entspricht das in etwa jeweils 
einem halben Rating-Punkt - ein Unterschied, der beide Male statistisch signifikant ist.

Dieser Positionseffekt hat zur Folge, dass sich die Differenzen zwischen Hk- und HgBildern je nach Abfolge nivellieren, oder zu einem ganzen Rating-Punkt addieren (Interaktionseffekt von Bewertung und Bildabfolge; s. folgende Tabelle und Grafik):

\begin{tabular}{|c|c|c|c|}
\hline & \multicolumn{3}{|c|}{ Attraktivitäts-Bewertungsdifferenzen nach Bildfolge } \\
\hline $\begin{array}{l}\text { Bild- } \\
\text { Nummer }\end{array}$ & $\begin{array}{c}\text { Mittelwert } \\
\text { 1. Stelle } \mathrm{Hk}->2 \text {. Stelle Hg }\end{array}$ & & $\begin{array}{c}\text { Mittelwert } \\
\text { 1. Stelle } \mathrm{Hg}->2 \text {. Stelle Hk }\end{array}$ \\
\hline 1 & $-0,95$ & $<$ & 0,10 \\
\hline 2 & $-0,80$ & $<$ & 0,25 \\
\hline 6 & $-0,95$ & $<$ & $-0,60$ \\
\hline 4 & 0,00 & $=$ & 0,10 \\
\hline 5 & $-2,15$ & $<$ & $-1,80$ \\
\hline 3 & $-1,90$ & $<$ & $-0,45$ \\
\hline 7 & $-1,05$ & $<$ & $-0,70$ \\
\hline 8 & $-1,55$ & $<$ & $-0,65$ \\
\hline 9 & $-0,20$ & $<$ & 0,70 \\
\hline 10 & $-0,85$ & $<$ & 0,95 \\
\hline 11 & $-0,30$ & $<$ & 0,20 \\
\hline 12 & $-0,65$ & $=$ & $-0,70$ \\
\hline 13 & $-0,90$ & $<$ & 0,10 \\
\hline 14 & $-1,35$ & $<$ & $-0,20$ \\
\hline 15 & $-2,25$ & $<$ & $-1,10$ \\
\hline 16 & 0,10 & $>$ & $-0,65$ \\
\hline \multirow[t]{3}{*}{$\begin{array}{c}\text { Alle } \\
\text { Bilder } \\
\end{array}$} & $-0,98$ & $<$ & $-0,28$ \\
\hline & $s=1,61 \quad M d n=-1,00$ & & $\mathrm{~s}=1,71 \quad \mathrm{Mdn}=0,00$ \\
\hline & $\mathrm{df}=638$ & , & $p<0,0001$ \\
\hline
\end{tabular}

Tab. 9: Übersicht der Differenzen in den Attraktivitätsbewertungen nach Präsentationsposition.

(Anmerkung: Bild 3 und 6 tauschen die Gruppenzugehörigkeit auf Grund stichprobenbedingter veränderter Schwere-Einschätzung) 
Grafisch wird dieser bisher geschilderte Sachverhalt noch einmal deutlich:

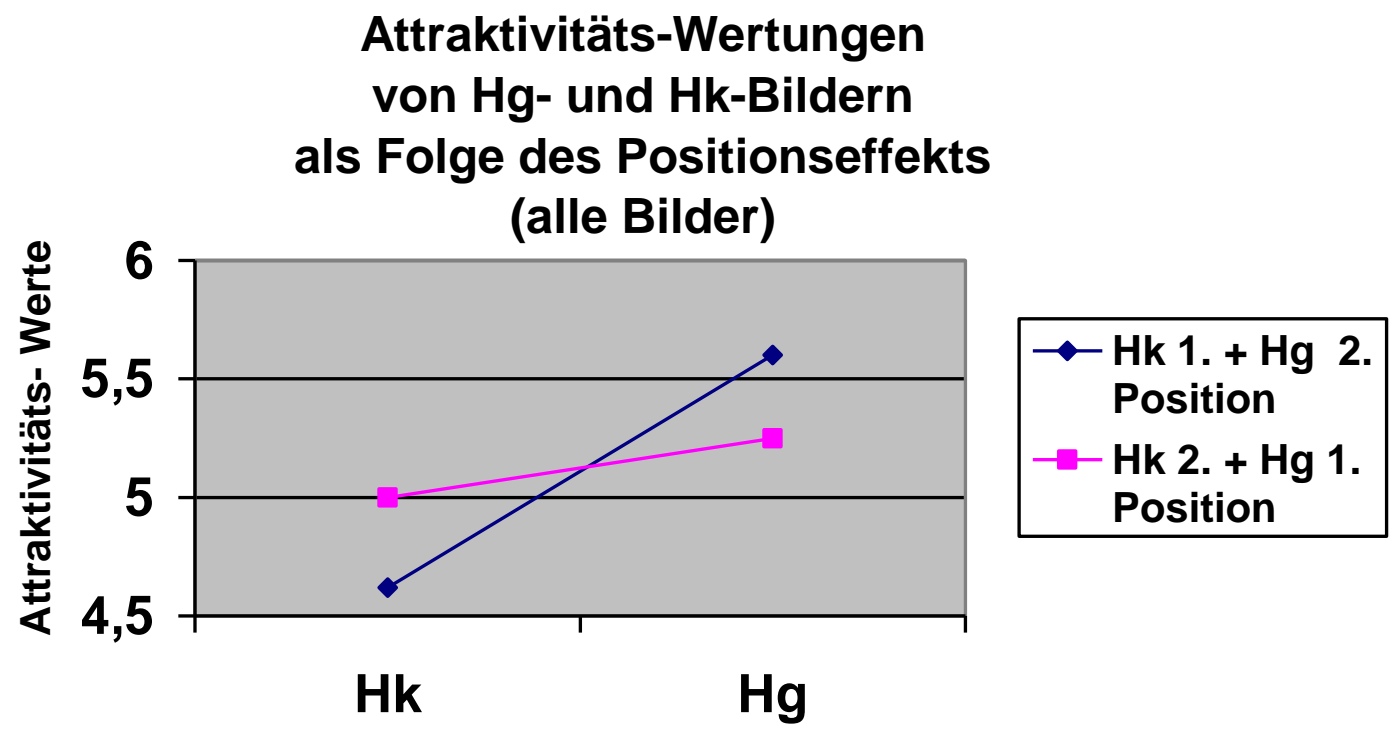

Abb. 14: Mittlere Attraktivitätswertungen von $\mathrm{Hk}$ - und $\mathrm{Hg}$-Bildern als Folge des Positionseffekts.

Der Einfluss des Positionseffektes als Bezugspunkt für die Urteilsbildung, lässt sich vereinfacht demnach so zusammenfassen:

Hat man jemanden als hautkrank kennengelernt, wirkt diese Person zu einem späteren Zeitpunkt - dann aber hautgesund - bedeutend attraktiver („schöner“); kennt man jemanden schon hautgesund, bewirkt eine Hautkrankheit lediglich eine minimale Änderung der anfänglichen Attraktivitäts-Bewertung.

Über alle Bilder und Probanden gesehen, lässt sich also ein Positionseffekt bei der Bewertung des Attributes „Attraktivität" feststellen. Je nach Bildabfolge hebt dieser in hochsignifikanter Weise Urteils-Differenzen nahezu auf $(\mathrm{Hg}->\mathrm{Hk})$, oder summiert sie auf etwa einen ganzen Wertungspunkt $(\mathrm{Hk}->\mathrm{Hg})$.

Bezüglich der Auffälligkeits-Bewertung zeigt sich, dass hautkranke Bilder höher eingestuft werden als ihre hautgesunden Pendants. Ein bedeutsamer Positionseffekt kann aber für das Attribut „Auffälligkeit” nicht festgestellt werden. Bei den hautgesunden Portraits ist es offensichtlich unentschieden, ob das Bild an erster Stelle auffälliger ist als dasselbe Bild an zweiter Stelle. Bei den Portraits in der hautkranken Version ist es scheinbar so, dass gehäuft (10 von 16) die 
entsprechenden Bilder an der ersten Stelle in der Präsentationsfolge als auffälliger bewertet werden - aber dieser Effekt erweist sich insgesamt als nicht signifikant.

\begin{tabular}{|c|c|c|c|c|c|c|}
\hline \multirow{3}{*}{$\begin{array}{c}\text { Bild- } \\
\text { Gruppe } \\
\text { Bild- } \\
\text { Nummer }\end{array}$} & \multicolumn{3}{|c|}{ Hk-Bild } & \multicolumn{3}{|c|}{ Hg-Bild } \\
\hline & 1. Stelle & & 2. Stelle & 1. Stelle & & 2. Stelle \\
\hline & Mittelwert-1 & & Mittelwert-2 & Mittelwert-1 & & Mittelwert-2 \\
\hline 1 & 5,8 & $>$ & 5,55 & 5,85 & $<$ & 6,15 \\
\hline 2 & 6,05 & $>$ & 5,6 & 5,15 & $<$ & 5,4 \\
\hline 3 & 5,4 & $>$ & 4,9 & 5,85 & $>$ & 5,0 \\
\hline 4 & 4,8 & $=$ & 4,8 & 4,75 & $=$ & 4,75 \\
\hline 5 & 6,75 & $>$ & 6,35 & 4,85 & $=$ & 4,8 \\
\hline 6 & 5,65 & $>$ & 5,35 & 5,00 & $=$ & 5,05 \\
\hline 7 & 5,7 & $=$ & 5,65 & 4,15 & $<$ & 4,45 \\
\hline 8 & 7,25 & $>$ & 6,65 & 4,95 & $>$ & 3,9 \\
\hline 9 & 3,75 & $<$ & 4,3 & 3,15 & $>$ & 2,85 \\
\hline 10 & 3,95 & $<$ & 4,65 & 3,25 & $<$ & 3,4 \\
\hline 11 & 4,1 & $=$ & 4,05 & 3,8 & $>$ & 3,5 \\
\hline 12 & 3,7 & $<$ & 3,95 & 4,4 & $>$ & 3,25 \\
\hline 13 & 4,25 & $>$ & 3,85 & 2,2 & $<$ & 3,2 \\
\hline 14 & 4,75 & $>$ & 3,85 & 2,85 & $=$ & 2,8 \\
\hline 15 & 7,1 & $>$ & 6,15 & 4,1 & $>$ & 3,85 \\
\hline 16 & 6,55 & $>$ & 6,25 & 3,7 & $>$ & 3,55 \\
\hline \multirow[t]{3}{*}{$\begin{array}{l}\text { Alle } \\
\text { Bilder }\end{array}$} & 5,35 & $>$ & 5,12 & 4,13 & $=$ & 4,09 \\
\hline & $\begin{array}{c}S=2,13 \\
M d n=5,00\end{array}$ & & $\begin{array}{c}S=2,12 \\
M d n=5,00\end{array}$ & $\begin{array}{c}S=2,08 \\
M d n=4,00\end{array}$ & & $\begin{array}{c}S=2,03 \\
M d n=4,00\end{array}$ \\
\hline & $d f=638$ & $t=1$ & $p=0,18$ & $d f=638$ & $\mathrm{t}=0$, & $p=0,85$ \\
\hline
\end{tabular}

Tab. 10: Bewertungen Attribut „Auffallend“ nach Präsentation an erster/zweiter Stelle. 
Auch grafisch wird dieser Sachverhalt noch einmal deutlich:

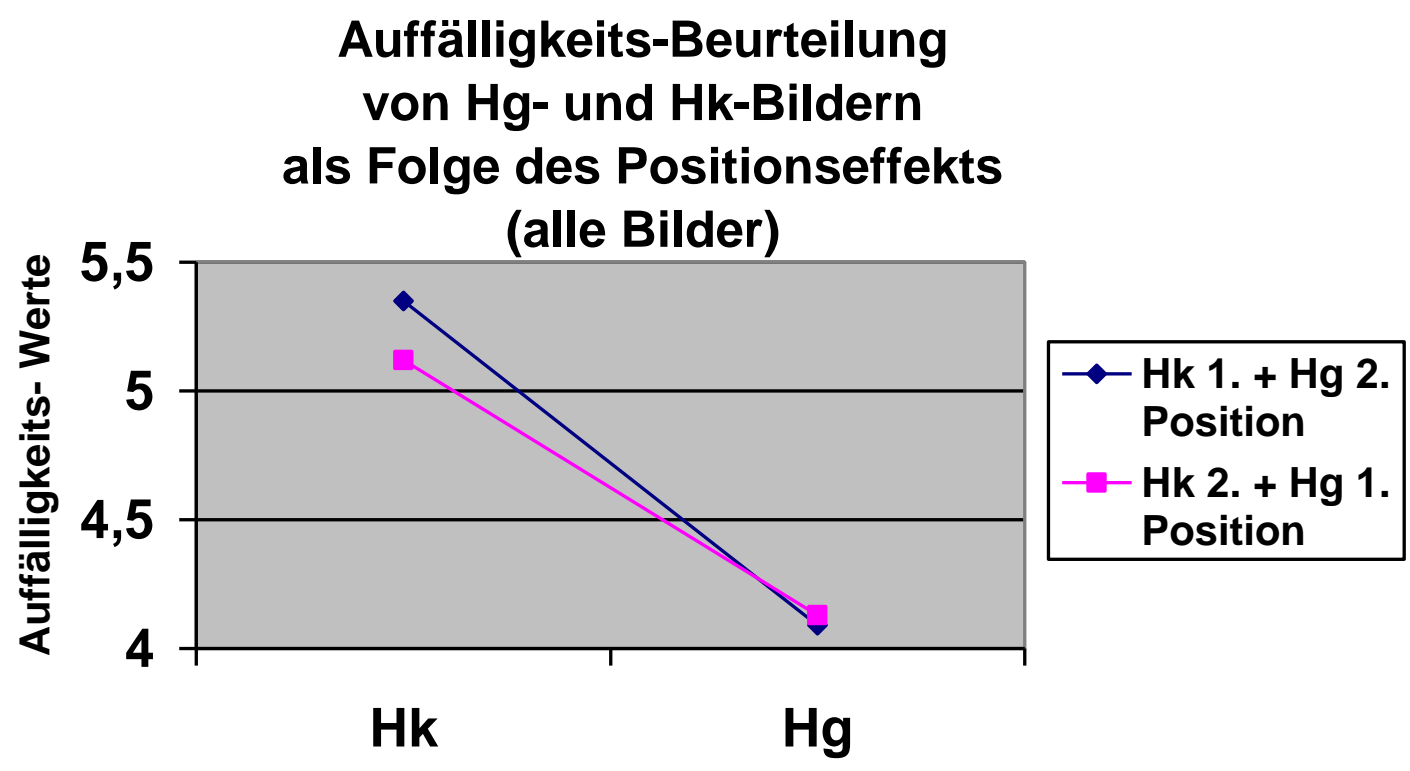

Abb. 15: Mittlere Auffälligkeitsbewertungen von $\mathrm{Hk}$ - und $\mathrm{Hg}$-Bildern im Präsentations-Positions-Vergleich.

Es lässt sich auch kein Interaktionseffekt von Bewertung und Bildabfolge sichern (s. folgende Tab. 11 und obige Abb. 12): 


\begin{tabular}{|c|c|c|c|}
\hline & \multicolumn{3}{|c|}{ Auffälligkeits-Bewertungsdifferenzen nach Bildfolge } \\
\hline $\begin{array}{l}\text { Bild- } \\
\text { Nummer }\end{array}$ & $\begin{array}{c}\text { Mittelwert } \\
\text { 1. Stelle Hk } \rightarrow \text { 2. Stelle Hg }\end{array}$ & & $\begin{array}{c}\text { Mittelwert } \\
\text { 1. Stelle } \mathrm{Hk}->\text { 2. Stelle Hg }\end{array}$ \\
\hline 1 & $-0,35$ & $=$ & $-0,30$ \\
\hline 2 & 0,65 & $>$ & 0,45 \\
\hline 3 & 0,4 & $<$ & 1,05 \\
\hline 4 & 0,45 & $>$ & 0,05 \\
\hline 5 & 1,95 & $>$ & 1,50 \\
\hline 6 & 0,60 & $>$ & 0,35 \\
\hline 7 & 1,25 & $<$ & 1,5 \\
\hline 8 & 3,35 & $>$ & 1,7 \\
\hline 9 & 0,9 & $<$ & 1,15 \\
\hline 10 & 0,55 & $<$ & 1,4 \\
\hline 11 & 0,6 & $>$ & 0,25 \\
\hline 12 & 0,45 & $>$ & $-0,45$ \\
\hline 13 & 1,05 & $<$ & 1,65 \\
\hline 14 & 1,95 & $>$ & 1,0 \\
\hline 15 & 3,25 & $>$ & 2,05 \\
\hline 16 & 3,0 & $>$ & 2,55 \\
\hline \multirow[t]{3}{*}{$\begin{array}{l}\text { Alle } \\
\text { Bilder }\end{array}$} & 1,25 & $>$ & 0,99 \\
\hline & $\mathrm{S}=2,23 \quad \mathrm{Mdn}=1,00$ & & $\mathrm{~s}=2,20 \quad \mathrm{Mdn}=1,00$ \\
\hline & \multicolumn{3}{|c|}{$d f=638 \quad t=1,48 \quad p=0,14$} \\
\hline
\end{tabular}

Tab. 11: Bewertungsdifferenzen des Attributs Auffälligkeit nach Präsentationsposition.

Nach diesen Urteils-Positions-Effekten steht nun zur weiteren Prüfung an, ob die beiden Bewertungs-Dimensionen „Emotion“ (Attraktivität) und „Kognition“ (Auffälligkeit) tatsächlich unabhängig voneinander sind, wie es in den einleitenden Abschnitten dieser Arbeit bereits angenommen wurde. Um dies abzusichern, wird die jeweilige Bewertungsdimension um noch jeweils einen Wertungsbegriff bzw. ein Attribut erweitert: Angenehmheit bzw. Ungewohntheit. Wenn also Unabhängigkeit zwischen den Dimensionen bestehen sollte, dann müssten einerseits Attraktivität und Angenehmheit bzw. Auffälligkeit und Ungewohntheit jeweils hoch interkorrelieren. 
Zwischen den Attributen verschiedener dimensionaler Begriffe dürfte andererseits keine bzw. eine lediglich sehr geringe Beziehung bestehen.

Bei einer derartigen Fragestellung unterzieht man in der Regel die Bewertungen und deren Interkorrelationsmatrix einer Faktorenanalyse (Varimax-Lösung, Rotieren der Ladungsmuster, SAS FACTOR Procedure). Es zeigt sich (Tab. 12), dass jeweils Attraktivität und Angenehmheit einerseits und Auffälligkeit und Ungewohntheit andererseits hoch auf jeweils einem Faktor laden und kaum bis gar nicht auf dem jeweiligen Gegenfaktor.

\begin{tabular}{|c|c|c|}
\hline Bewertungen & Faktor-1 & Faktor-2 \\
\hline Attraktivität & 0,10 & $\mathbf{0 , 9 0}$ \\
\hline Angenehmheit & $-0,02$ & $\mathbf{0 , 9 0}$ \\
\hline Auffälligkeit & $\mathbf{0 , 9 2}$ & 0,14 \\
\hline Ungewohntheit & $\mathbf{0 , 9 3}$ & $-0,06$ \\
\hline
\end{tabular}

Tab. 12: Faktorladungen von emotionalen und kognitiven Bewertungen.

Sehr ähnlich sieht es aus, wenn man Sekundärwerte - nämlich die Differenz der jeweiligen Bewertung zwischen dem hautkranken und dem hautgesunden Bild einer Faktorenanalyse unterzieht.

\begin{tabular}{|c|c|c|}
\hline $\begin{array}{c}\text { Bewertungs- } \\
\text { Differenz } \\
\text { Hk - Hg }\end{array}$ & Faktor-1 & Faktor-2 \\
\hline Attraktivität & $-0,03$ & $\mathbf{0 , 8 7}$ \\
\hline Angenehmheit & $-0,03$ & $\mathbf{0 , 8 7}$ \\
\hline Auffälligkeit & $\mathbf{0 , 8 8}$ & 0,07 \\
\hline Ungewohntheit & $\mathbf{0 , 8 7}$ & $-0,13$ \\
\hline
\end{tabular}

Tab. 13: Faktorladungen bezüglich der Bewertungsdifferenzen. 
Möchte man den dahinter stehenden Sachverhalt in Korrelationen ausgedrückt sehen, so ist die folgende Tabelle informativ:

\begin{tabular}{|c|c|c|c|c|c|c|}
\hline $\begin{array}{c}\text { Be- } \\
\text { wertung }\end{array}$ & $\begin{array}{c}\text { attraktiv } \\
- \\
\text { angenehm }\end{array}$ & $\begin{array}{c}\text { attraktiv } \\
- \\
\text { auffallend }\end{array}$ & $\begin{array}{c}\text { attraktiv } \\
- \\
\text { ungewohnt }\end{array}$ & $\begin{array}{c}\text { angenehm } \\
- \\
\text { auffallend }\end{array}$ & $\begin{array}{c}\text { angenehm } \\
- \\
\text { ungewohnt }\end{array}$ & $\begin{array}{c}\text { auffallend } \\
- \\
\text { ungewohnt }\end{array}$ \\
\hline absolut & $\mathbf{0 , 6 4}$ & 0,22 & 0,02 & 0,08 & $-0,02$ & $\mathbf{0 , 7}$ \\
\hline & & & & & & \\
\hline Differenz & $\mathbf{0 , 5 3}$ & 0,03 & $-0,11$ & 0,01 & $-0,1$ & $\mathbf{0 , 5 4}$ \\
\hline
\end{tabular}

Tab. 14: Interkorrelationen der Attribute.

Sehr ähnliche Korrelationswerte ergeben sich, wenn man zunächst für jeden einzelnen Probanden die Bewertungen interkorreliert und diese dann mittelt.

Damit ist nun abgesichert, dass Attraktivität und Auffälligkeit unabhängige Bewertungen mit sehr unterschiedlichen Bedeutungshöfen darstellen.

\subsubsection{Hypothesen-Testung: Korrelationen zwischen Stigma-Areal- Fixationszeit und Bewertungen}

Neben der Frage nach der methodischen Eignung des RFV-Ansatzes ist das zentrale inhaltliche Anliegen dieser Arbeit die Frage, inwieweit Stigma-Fixationszeiten in der Tat ein Indikator für emotionale Bewertung (Attraktivität) sind (hautkranke Personen sprechen pejorativ vom Anstarren in dem Sinne, dass das Fixieren von sozialer Abwertung begleitet sei) - oder ob das Fixieren nicht doch eher ein Zeichen für kognitive Verarbeitung von schemafremden Auffälligkeiten und Ungewohntheiten darstellt.

Zur Klärung werden Korrelationen zwischen Bewertungen und Blickzeiten nach der p-Technik (Variationen über die Bilder; für jeden einzelnen Probanden; über z-Werte dann für alle Probandinnen gemittelt; s. Näheres im Methodenteil) berechnet. Allerdings erscheinen Partial- und getrennte Korrelationen angebracht zu sein, denn zwei Einflussgrößen gilt es zu neutralisieren: 
- den Präsentations-Positionseffekt: ob ein Bild an erster oder zweiter Stelle gezeigt wurde, hat Einfluss (wie oben gezeigt) sowohl auf Blickzeiten als auch auf Bewertungen (besonders Attraktivität)

- die Basis-Attraktivität bzw. -Auffälligkeit: „Attraktive“ werden in dieser Eigenschaft der Attraktivität durch ein Stigma anders beeinflusst als „Unattraktive“ und Entsprechendes gilt dann auch für „Auffällige“ und „Unauffällige“.

Um diesen Weg deutlich zu machen, werden alle relevanten Korrelationswerte - d.h. deren Mittelwerte - in der folgenden Tabelle wiedergegeben:

\begin{tabular}{|c|c|c|c|c|c|c|c|c|c|c|c|}
\hline \multirow[b]{2}{*}{ FZ (Stigma) } & \multicolumn{2}{|c|}{ einfach } & \multicolumn{2}{|c|}{$\begin{array}{l}\text { partial } \\
\text { "Basis“ }\end{array}$} & \multicolumn{2}{|c|}{$\begin{array}{l}\text { partiell } \\
\text { 1.Stelle }\end{array}$} & \multicolumn{2}{|c|}{$\begin{array}{l}\text { partiell } \\
\text { 2.Stelle }\end{array}$} & \multicolumn{3}{|c|}{$\begin{array}{c}\text { Gesamt partiell } \\
1 .+2 . \text { Stelle }\end{array}$} \\
\hline & r & $z$ & $r$ & z & $r$ & z & r & z & $r$ & $\mathbf{z}$ & $\%$ \\
\hline Attraktiv &,- 24 &,- 24 &,- 28 &,- 29 &,- 35 &,- 37 &,- 33 &,- 35 & ,35 &,- 37 & 12 \\
\hline Angenehm &,- 10 &,- 10 &,- 09 &,- 09 &,- 12 &,- 12 &,- 07 &,- 07 &,- 09 &,- 09 & 1 \\
\hline Auffallend & ,32 & ,33 & ,37 & ,39 & ,45 & ,48 & ,47 & ,51 & ,45 & ,49 & 20 \\
\hline Ungewohnt & ,30 & ,31 & ,32 & ,33 & ,37 & ,38 & ,35 & ,36 & ,37 & ,38 & 14 \\
\hline
\end{tabular}

Tab. 15: Unterschiede in den Partialkorrelationen bei unterschiedlicher Präsentationsposition.

(Anmerkung: Neben den Mittelwerten ist eigentlich die Streuung der Werte über die Probandinnen noch von Interesse. Diese wird aus Prägnanzgründen für die Kernaussage hier nicht erwähnt.)

Es zeigt sich, durch Auspartialisierung der jeweiligen Basis-Eigenschafts-Bewertung im Hg-Bild und Differenzierung gemäß der Präsentationsposition, eine Steigerung im Ausmaß der Korrelation zwischen Blickzeit und der jeweiligen Bewertung.

Insgesamt lässt sich nun bezüglich der Hypothesen 3a, 3b und 3c sagen:

- Hypothese 3a „Stigma-Fixationszeit korreliert kaum mit emotionaler Bewertung": Auch wenn länger auf das Stigma-Areal geschaut wird, fallen die Beurteilungen nur geringfügig „unattraktiver“ aus - zusammenfassende mittlere 
Korrelation beträgt $r=-0,35$, was bedeutet, dass damit nur $12 \%$ der gemeinsamen Varianz erfasst ist. Dieser Wert ist zwar statistisch signifikant von Null (kein Zusammenhang) verschieden, aber inhaltlich als eher klein einzustufen. Dass emotionale Bewertung und Fixationszeit für Stigmata nur sehr wenig zusammenhängen, wird zudem durch die nahezu Null-Korrelation $(r=-0,09)$ zwischen „angenehm“-Bewertungen und Stigma-Fixationszeiten unterstrichen.

- Hypothese 3b "Stigma-Fixationszeit korreliert mit kognitiver Bewertung“. Je länger auf das Stigma-Areal geschaut wird, desto „auffälliger“ werden die Bilder beurteilt - zusammenfassende mittlere Korrelation beträgt $r=-0,45$, was $20 \%$ der gemeinsamen Varianz erfasst. Dieser statistisch hoch signifikante Wert weist auf einen bedeutsamen Zusammenhang zwischen Stigma-Fixationszeit und kognitiver Bewertung hin. Gestützt wird dies durch die entsprechenden Korrelationen mit den „ungewohnt"-Urteilen - diese liegen bei etwa 0,35 - kleiner, aber dennoch signifikant.

- Hypothese 3c „Stigma-Fixationszeit korreliert mit kognitiver Bewertung signifikant höher als mit emotionaler Bewertung". Gemessen an der durch die Korrelation jeweils aufgeklärten Varianz, ist mit 20\% der Zusammenhang zwischen Blickzeit und Auffälligkeit beinahe doppelt so hoch wie der zwischen Blickzeit und Attraktivität von $12 \%$. Dennoch weist ein t-Test für die Mittelwerte aus den Differenzen der z-Werte für die Korrelationen Stigma-FZ mit kognitiver Bewertung versus Stigma-FZ mit emotionaler Bewertung einen nicht signifikanten Wert auf:

$M_{(z-\text { Diff })}=0,13 / s_{(z-\text { Diff })}=0,52 / t=1,55 / p=0,13$. Sicher gibt es einen signifikanten Unterschied zwischen kognitiver und emotionaler Bewertung, wenn die ,angenehm'Urteile hier benutzt werden. Trotzdem kann die Vorstellung einer „AnstarrAbwertung" hautkranker Probandinnen aufgrund dieser Ergebnisse nicht komplett zurückgewiesen werden, da ein gewisser - wenn auch schwächerer - Zusammenhang zwischen der Blickzeit und der emotionalen Bewertung besteht.

Zusammenfassend belegen die Daten, dass Fixationszeiten für Stigmata nur bedingt und begrenzt etwas mit emotionalen Bewertungen gemein haben. Weitaus sicherer ist der Zusammenhang mit kognitiven Bewertungen. Damit zeichnet sich ein Faktum $a b$, das mit den gängigen Vorstellungen Hautkranker, die sich angestarrt und damit 
sozial abgewertet fühlen, unvereinbar ist - und dadurch einen neuen Ansatz für Beratungen und Psychotherapie darstellen kann. 


\section{Diskussion}

Im folgenden Abschnitt sollen die Ergebnisse der vorliegenden Arbeit im Kontext mit anderen Arbeiten sowie in Bezug auf Validität und Methodik diskutiert werden. Dabei wird nicht erneut auf methodische Einzelheiten eingegangen, sondern der Fokus auf die Auswertung der Hypothesen gelegt. Abschließend erfolgt ein Ausblick auf zukünftige Arbeiten im Bereich der Blickregistrierung und Attraktivitätsforschung.

\subsection{Validitätsprüfung}

\subsubsection{Realitätsnähe des RFV}

Wie bei allen experimentellen Arbeiten stellt zunächst die Übertragbarkeit in „reale Interaktionen" ein ganz entscheidendes Kriterium für die Validität der Untersuchung dar. Die Studie fand ausschließlich am Laptop statt. Auf mögliche Unterschiede der Umgebungswahrnehmung in der Realität und am Bildschirm wiesen Foulsham et al. (2011) hin. Zwar kann der Restricted Focus Viewer kaum in realen Interaktionen eingesetzt werden, aber er weist, wie zuvor beschrieben (Abschnitt 1.4), eine hohe Korrelation mit hoch validen Eye-Tracking-Systemen auf. Zusätzlich wurde mit Hilfe der Instruktion und Integration der Untersuchungsbilder in eine "Freizeitszenerie“ (Café) versucht, die Probandinnen gedanklich auf eine einheitliche reale Situation zu beziehen. Es ist vorstellbar, dass bei der Wahl eines anderen Bezugsrahmens schon implizit Erwartungen an das Erscheinungsbild geknüpft werden (beispielsweise 'besonders hübsch' in Discos oder 'elegant' in Restaurants), die sich dann auch in anderen RFV-Ergebnissen niederschlagen könnten.

\subsubsection{Effektivität der Blickregistrierung}

Einen wichtigen Faktor in der Blickregistrierung stellte die Größe des mausgesteuerten Fokus-Fensters dar. Das Fokus-Fenster entsprach in gewisser Weise den „Augen“ durch welche die Versuchsteilnehmerinnen die Portraitfotos betrachteten und bildete somit eine entscheidende Grundlage für die Auswertung der 
Blickregistrierung. Jansen et al. (2003) wiesen darauf hin, dass eine zu starke Einschränkung der Fokusfenstergröße zu einer Beeinträchtigung des natürlichen Blickverhaltens führen würde. Darüber hinaus musste die Fenstergröße in geeigneter Relation zu den definierten Sektoren stehen, die ein bestimmtes Merkmal repräsentierten (z.B. Wange, Stirn, Nase, Mund,..., vgl. Abb. 8). Die große Bedeutung dieses Verhältnisses unterstrichen auch Bednarik und Tukiainen (2005), die bei abnehmender Fokus-Fenstergröße zwar einen Anstieg der Anzeigegenauigkeit bestätigten, jedoch ein gleichzeitig zunehmendes Abweichen von den natürlichen Suchstrategien der Probanden beschrieben haben.

Konkrete Auswahlkriterien dieser Parameter wurden bereits im Abschnitt 2.3 erläutert. Dabei war beispielsweise für die Wahl der Fenstergröße von entscheidender Bedeutung, dass nicht zwei Merkmale gleichzeitig fixiert werden konnten, um differenzierter auswerten zu können.

In diesem Zusammenhang fielen die Ergebnisse im Hinblick auf die Selbstbeurteilung der Probandinnen im Rahmen der Evaluation des RFV-Verfahrens zur GrößenEinschätzung des Fokus-Fensters aber eher zwiespältig aus. 60\% der Probandinnen empfanden das zur Verfügung gestellte Mausfenster als eher zu klein.

Wenn man, trotz dieser Kritik, dann jedoch auf andere objektive Messwerte schaute, ließen sich viele grundlegende Erkenntnisse der Blickregistrierung mit dem verwendeten RFV-Verfahren nachweisen. So erhielten beispielsweise die Augen bei der Portraitfoto-Betrachtung die meiste Aufmerksamkeit aller Gesichtspartien, gefolgt vom Mund und danach Partien wie Nase und Kinn (vgl. Luria und Strauss 1978). Es konnte darüber hinaus ein so genannter left gaze bias (Guo et al. 2009) aufgezeigt werden. Danach kommt bei der Betrachtung von Bildern, dem linken visuellen Feld und damit der rechten Seite des betrachteten Objekts oder der betrachteten Person eine verstärkte Aufmerksamkeit im Vergleich zur Gegenseite zu, unabhängig von der Aufgabenstellung (Guo et al. 2012).

Somit kann davon ausgegangen werden, dass sich die RFV-Technik im Hinblick auf die Blickregistrierung bewährt hat. Die von etwa der Hälfte der Probandinnen als eingeschränkt empfundene Sicht durch das Mausfenster lässt sich u.a. durch die erstmalige, ungewohnte Nutzung der Technik erklären, die trotz der drei Übungsbilder vielleicht noch nicht als "normales Sehen“ empfunden wurde.

Neben einer Erhöhung der Übungsbilderanzahl könnte eine mögliche Verbesserung des optischen Eindrucks durch die Verwendung eines abgestuften Fokus-Fensters 
mit mehreren Kontrastzonen erreicht werden. Auf Einzelheiten solch eines unterteilten Fensters wird im Abschnitt (4.2.2) genauer eingegangen.

\subsubsection{Eignung der Fotonegativ-Filterung}

Ein wesentliches Element der RFV-Technik ist eine angemessene Filterung des präsentierten Bildes - in dieser Studie wurde hierfür eine Überlagerung des Originalbildes durch ein Negativbild verwendet. Das Ziel dieser Filterung war, wie bereits zuvor beschrieben, ein Maximum an diagnostischer Information, welche zum Bearbeiten der Fragen relevant ist, im gefilterten Bereich zu blocken, aber gleichzeitig ein Maximum an navigatorischer Information, die Aufschluss über die allgemeine Beschaffenheit des Portraitfotos und die Lage der diagnostischen Information gibt, bereitzustellen. Die Wichtigkeit dieser Filtereigenschaften stellten schon Jansen et al. (2003) als sehr wichtigen Faktor heraus.

Wie zuvor in Abschnitt 2.3.3 erläutert wurde, kann es bei der Konvertierung ins Fotonegativ zu Farb- und möglichen Kontrastveränderungen kommen. Ho-Phuoc et al. (2012) konnten allerdings zeigen, dass etwaige Farbunterschiede keine Änderung des Fixationsverhaltens der Betrachter bewirken.

Mitentscheidend für die Aussagekraft des Tests und der Ergebnisse ist also die Wirksamkeit des Filters. Dieses Kriterium unterstrichen auch Jones und Mewhort (2004) in ihrer Arbeit. Sie betonten, dass die Fokus-Window-Technique besonders wirkungsvoll sei, wenn die diagnostische Information durch den Filter höchstens auf dem Level der Zufallswahrscheinlichkeit beurteilt werden kann.

In den Aussagen der Selbstbeurteilung waren sich alle Teilnehmerinnen ausnahmslos darüber einig, dass eine Einschätzung der Personen auf den Portraitfotos allein auf Grund des Negativs für sie nicht möglich gewesen wäre. Der Negativfilter hat seinen Zweck somit offensichtlich vollkommen erfüllen können. Jones und Mewhort (2004) wiesen ebenfalls auf die Möglichkeit einer abweichenden Selbsteinschätzung der Filterung hin, also eine die von den Probandinnen zwar als "gut“ empfunden wurde, bei Prüfung objektiver Kriterien jedoch zeigt, dass sie nicht effektiv genug war.

Angesichts der nachweislich veränderten Blickzeiten und der erfolgreichen $\mathrm{Re}$ produktion wichtiger objektiver Basisphänomene der Blickregistrierung (vgl. Abschnitt 3.2) kann die Filterung mittels Fotonegativ in diesem Zusammenhang als effektive und sinnvolle Methode gewertet werden. 


\subsubsection{Auswirkungen der Expositionszeit}

Die für die Betrachtung der Experimentalfotos gewählte Zeit von 30 Sekunden hatte ihren Grund. Voruntersuchungen und auch Selbsttests ergaben, dass die Dauer an die Größe der Portraitfotos und des Mausfensters adaptiert werden müssen. O'Reilly und Plamondon (2011) bescheinigten der Kopplung von Maus und visueller Aufmerksamkeit eine hohe Reliabilität in ihrer Arbeit. Ziel in dieser Studie war, keinen Zeitdruck auf die Teilnehmerinnen auszuüben, um einen möglichen Einfluss auf die Betrachtungsstrategien zu verhindern. Betrachtungszeiten unter 30 Sekunden wurden als zu kurz befunden. Die in der anschließenden Befragung mitgeteilte Einschätzung von $90 \%$ der Probandinnen, die Zeit von 30 Sekunden sei ausreichend gewesen, bestätigte die Auswahl der Dauer.

Andererseits konnten deutliche Differenzen im zeitlichen Verlauf beim Betrachten der Portraitfotos aufgezeigt werden. Stigma-Areale wurden nämlich länger in den ersten 15 Sekunden der Präsentation als in der darauf folgenden gleichen Zeit betrachtet (vgl. Tab. 4 und Tab. 5). Dieser Befund ist keinesfalls als methodisches Artefakt zu deuten, sondern bestätigt einen ähnlichen Zusammenhang wie inn schon Brüninghaus (1980), Kahle (2007) und auch Nummenmaa et al. (2006) beschrieben, obwohl sie zum Teil mit anderen Techniken (z.B. Eye Mark Recorder) oder auch kürzerer Darbietungszeit arbeiteten.

\subsubsection{Einfluss des Bildmaterials}

Im Abschnitt 2.1 wurden Ein- und Ausschlusskriterien für das verwendete Bildmaterial besprochen. Leonards und Scott-Samuel (2005) beschrieben in ihrer Arbeit den Einfluss interindividueller Unterschiede, die im Blickverhalten von Testpersonen auftraten, sofern innen die Bilder vertraut waren. Es war daher wichtig, dass die Probandinnen keine innen bekannten Personen und Fotos betrachteten. Ein möglicher Einfluss von internalen Faktoren musste in Betracht gezogen werden (vgl. auch Chun et al. 2011). Neben den eigenen Vorerfahrungen und Erinnerungen gehört dazu u.a. auch der Aufmerksamkeitszustand der Probandinnen (vgl. Noton und Stark 1971). 
Darüber hinaus wurde in Anlehnung an die Untersuchung von Nummenmaa et al. (2006), besonderer Wert auf eine möglichst neutrale Mimik der Personen auf den untersuchten Portraitfotos gelegt. Diese Autoren bescheinigen in ihrer Studie emotionalen Bildern einen aufmerksamkeitssteigernden Einfluss. Auswirkungen dieses so genannten happy-face advantage (Mack and Rock 1998; Leppänen und Hietanen 2003) auf die Ergebnisse der Studie sollten möglichst vollständig vermieden werden, da u. a. auf Attribute wie „Attraktivität“ oder Einschätzungen wie „angenehm" hin untersucht wurde.

Es sei an dieser Stelle jedoch nochmals ausdrücklich darauf hingewiesen, dass diese Effekte keinesfalls spezifische Probleme der RFV-Technik darstellen, sondern allgemein bei Blickregistrierungsuntersuchungen, unabhängig von der Messtechnik, Bestandteil der Überlegungen sein sollten.

\subsubsection{Spezielle Zusammenstellung der Versuchsgruppe}

Die untersuchte Gruppe wurde in Alter und Geschlecht bewusst und gezielt eingeschränkt. Diese Limitierung bezieht sich u. a. auf die Erkenntnisse von Hewig et al. (2008), die Blickregistrierungsdifferenzen im Hinblick auf das Geschlecht des Betrachters und Betrachteten zeigten. Eine solche Einschränkung scheint auch in zukünftigen Studien notwendig zu sein, um konkrete Ergebnisse gewinnen zu können (vgl. Gegenfurtner et al. 2011). Denn trotz aller Einschränkungen sind auch in dieser Studie Einflussfaktoren vorstellbar, die z.B. in Abhängigkeit von eigenen Vorerfahrungen der Teilnehmerinnen mit Hautkrankheiten aufgetreten sein könnten (vgl. Stangier et al. 2008). Im Hinblick auf die Ergebnisse des Fragenkataloges, wonach ca. $40 \%$ der Probandinnen selbst, aktuell oder zuvor, schon einmal von einer Hautkrankheit betroffen waren, ist solch ein Effekt nicht gänzlich auszuschließen.

\subsection{Vergleich der RFV-Technik mit Eye-Tracking-Verfahren}

In diesem Abschnitt sollen nun die Vor- und Nachteile der Focus-Window-Technik (FWT) im Gegensatz zu Eye-Tracking-Systemen (ETS) besprochen werden und erläutert werden, welches Verfahren wann als vorteilhafter erscheint. 


\subsubsection{Vor- und Nachteile}

Bei Untersuchungen mit ETS muss das Probandenkollektiv im Vergleich zur RFVTechnik stärker eingeschränkt werden. Dies ergibt sich zunächst einmal aus dem Funktionsprinzip, welches den ETS zugrunde liegt, bei dem ein an der Hornhaut des Auges reflektierter Lichtstrahl erfasst und ausgewertet wird. Dadurch sind von vorn herein Brillen- und Linsenträger für etwaige Untersuchungen ungeeignet. Zudem ist mit der Verwendung von ETS ein höherer technischer und auch finanzieller Aufwand verbunden (Jansen et al. 2003). Diese Systeme sind zumeist an einen festen Ort gebunden und verlangen so von den Untersuchungsteilnehmern eine höhere zeitliche Flexibilität und räumliche Mobilität (vgl. Kahle 2007), wohingegen sich diese Untersuchung durch die Verwendung des Laptops sehr flexibel gestalten ließ. Bei Verwendung eines Eye Mark Recorder (EMR), den z.B. Brüninghaus (1980) nutzte, oder vergleichbarer Apparaturen muss darüber hinaus eine Messvorrichtung am Kopf des Probanden befestigt, oder dieser selbst fixiert werden. Dies stellt nicht nur eine weitere Einschränkung für das potentielle Untersuchungskollektiv dar, sondern limitiert natürlich auch in gewisser Weise die Realitätsnähe und das Ziel die untersuchten Personen gedanklich möglichst neutral bezüglich etwaiger experimenteller Zwänge und der Untersuchungssituation zu halten. Eine befestigte Messvorrichtung kann außerdem zu Einschränkungen bei der Untersuchungszeit führen. Hinzu kommt die Notwendigkeit, die ETS für den jeweiligen Probanden einzeln kalibrieren zu müssen, um die Genauigkeit beim Versuchsablauf sicherzustellen. Dazu müssen sie gegebenenfalls auch während der Untersuchung rekalibriert werden. Es ist möglich, diesen experimentellen Anforderungen mit modernen Remote Eye-Trackern, welche die Blickrichtungen per Kamera ohne Befestigungen am Probanden erkennen, zumindest teilweise zu entgehen. Risko und Kingstone (2011) zeigten in ihrer Arbeit jedoch auch bei Eye-Tracking-Verfahren eine Art soziale Induktion durch "Video-Überwachung" auf. Darüber hinaus nimmt auch bei diesen Systemen, im Gegensatz zur FWT, die Anzeigegenauigkeit zur Peripherie hin mit Sicherheit ab. Dies beruht u.a. auf einer nicht exakt sphärischen Krümmung der Hornhaut (Brüninghaus 1980).

Doch auch die RFV-Technik bringt Einschränkungen mit sich. So ist die Anzeigegenauigkeit zwar in allen Bereichen gleich hoch, aber zugleich stark abhängig von den zuvor im Abschnitt 2.3 besprochenen Faktoren, wie beispielsweise der Größe 
des mausgesteuerten Fokus-Fensters. Ebenso kann sich der Blick des Probanden zu bestimmten Zeiten auch außerhalb des Fokus-Fensters befinden, wie Jansen et al. (2003) problematisierten. Ein kurzzeitiges Abschweifen des Blickes vom FokusFenster oder gar Monitor lässt sich natürlich nur schwer bis gar nicht verhindern oder registrieren. Als weiteren Faktor gab u.a. Blackwell (2000) zu bedenken, dass beim RFV-Verfahren die natürlichen Blickbewegungszeiten teilweise durch neue Zeitgrößen für „Hand-Auge-Koordination“ ersetzt werden. Dies ist speziell bei Aussagen über Geschwindigkeiten zu berücksichtigen. Von entscheidender Bedeutung für die Ergebnisse der FWT ist eine an die Fragestellung adaptierte Kalibrierung des Filters (Jones und Mewhort 2004), die bei ETS nicht existiert. Nur mit der Bildfilterung kann sichergestellt werden, dass lediglich der scharfe Mausfensterbereich beurteilt werden kann und nicht auch die „parafoveal“ gelegene Umgebung. Doch genau an dieser Stelle ergibt sich eine Schwachstelle der ETS. Brüninghaus (1980) zufolge können die ETS nur ungenügend abbilden, was die Probanden auch mit der Parafovea an relevanten Informationen registrieren, ohne diese explizit zu fixieren. Die visuelle Wahrnehmung des Menschen erfolgt nämlich nicht ausschließlich punktförmig mit der Fovea, wie es die ETS registrieren.

\subsubsection{Schlussfolgerungen}

Es lässt sich also festhalten, dass die menschliche Wahrnehmung weder rechteckig, wie es die RFV-Technik simuliert, noch punktförmig, wie es die ETS suggerieren, erfolgt (vgl. Nuthmann und Henderson 2010). Beide Verfahren sind dennoch brauchbar, wenn es um bestimmte Fragestellungen in der Blickregistrierung geht. Sie sollten allerdings unter Berücksichtigung ihrer Schwachstellen für zukünftige Studien verbessert werden.

Einen guten Verbesserungsvorschlag für die FWT bietet Blackwell (2000) in seiner Arbeit an. Dabei wird eine scharfe Grenze zwischen detaillierter Wahrnehmung und "Gefiltertem" vermieden. Solch eine Grenze birgt laut Blackwell (2000) die Gefahr einer zu starken Kontrastbildung und führt dadurch zu einer intensiveren Verarbeitung des Sichtfeldes. Indem das Fokus-Fenster in mehrere Zonen unterteilt wird, analog zur Abnahme des Auflösungsvermögens im menschlichen Sehen zur Peripherie hin, könnte man diesen Kontrast relativieren. 


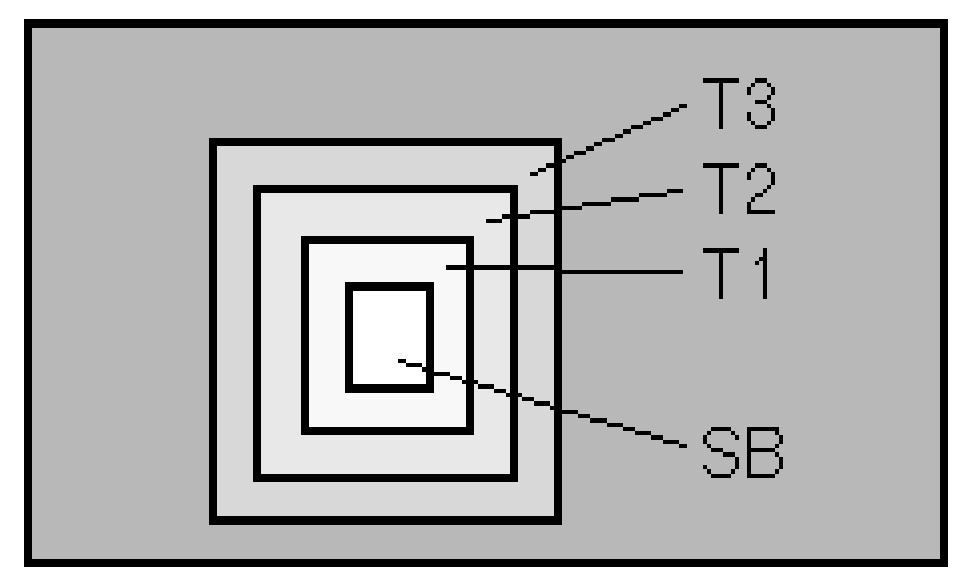

Abb. 16: Sichtbereich mit Zwischenstufen (Blackwell 2000, S.4).

Der Bereich SB stellt in diesem Modell den Bereich dar, welcher vollständig scharf zu erkennen ist. In den Bereichen T1-T3 wird das Versuchsbild zunehmend "gefiltert", also geringer aufgelöst. Dadurch wird der Übergang weicher und der Wahrnehmungseindruck natürlicher.

Beim direkten Vergleich der Messmethoden stellte jedoch auch Blackwell (2000) fest, dass es zwischen den mit der EMR-Technik und den mit der RFV-Technik gewonnenen Daten keine signifikanten Unterschiede gab.

Hinsichtlich der Validität der RFV-Technik werden auch in Zukunft die adäquate Kalibrierung des Filters an die Aufgabenstellung und die richtige Relation des FokusFensters zur Bildgröße die entscheidenden Faktoren sein.

Abschließend lässt sich also feststellen, dass beide Verfahren Vor- und Nachteile mit sich bringen, die es bei der Auswahl der Messtechnik zu berücksichtigen gilt. So müssen Untersuchungsobjekte bei der FWT, die günstiger und flexibler ist, immer angemessen gefiltert werden, während die ETS eine genaue und oftmals wiederholte Kalibrierung erfordern. Deshalb wird in Einklang mit Jones und Mewhort (2004) eine Anpassung der Untersuchungsmethode an die Aufgabenstellung empfohlen. Geht man der Fragestellung nach, wie ein Portraitfoto oder eine Szene erfasst wird, im Sinne von Blickpfaden, dann empfiehlt es sich eher die ETS zu nutzen. Möchte man jedoch Aussagen über Zeit-Informationen machen, die zu einer Bewertung von Bildern oder Szenen herangezogen werden, dann ist das RFV-Verfahren bei geeigneter Filterung eine sehr valide Messtechnik. 


\section{3 Überprüfungen der aufgestellten Hypothesen}

In diesem Abschnitt sollen die Ergebnisse der zuvor aufgestellten Hypothesen geprüft und im Kontext anderer Studien und Erkenntnisse im Forschungsgebiet der Blickregistrierung diskutiert werden.

Die erste Hypothese bezog sich auf die Brauchbarkeit des RFV für das Registrieren von Blickverhalten. Ein ganz zentraler Punkt bestand dabei in der Reproduzierbarkeit von Ergebnissen, die bei vorangegangenen Eye-Tracking- und RFV-Studien gewonnen wurden. So konnte, wie schon zuvor bei Kahle (2007) mit dem RFV oder auch bei Brüninghaus (1980) mit dem EMR, ein statistisch signifikanter Aufmerksamkeitsanstieg für Gesichtsareale mit Hautveränderungen nachgewiesen werden. Dieses veränderte Blickverhalten bewirken sowohl eher leichte, als auch als schwerer einzustufende Veränderungen. Jedoch lässt sich aus den Ergebnissen folgern, dass der Effekt umso stärker ausfällt, je schwerer die Hauterkrankung eingeschätzt wird. Zugleich wiesen die als hautgesund präsentierten Portraitfotos vergleichbare Blickverteilungsmuster auf, wie man sie beispielsweise bereits aus den Studien von Luria und Strauss (1978) kennt. Diese Faktenlage würde erst einmal für einen reizgesteuerten Prozess sprechen (vgl. Nuthmann und Henderson 2010).

Die zeitlich veränderte Gewichtung in der Betrachtung von Gesichtern muss anscheinend bei den Betroffenen zu einem Gefühl des „Anstarrens“ führen mit gleichbedeutender Ablehnung und Abwertung durch den Interaktionspartner wie es beispielsweise auch Schubert (1989), Allesch (2006) oder Rumpsey und Harcourt (2004) beschrieben haben. Um diesen negativen Verknüpfungen eine Alternative entgegenzusetzen, war es von besonderer Wichtigkeit, nach Indikatoren zu forschen, welche Hinweise für eine anderweitige Deutung des veränderten Blickverhaltens etwa im Sinne eines kognitiven Schema-Konzept-Ansatzes (Cash (1989); Henss (1998); Murray et al. 2003; Garcia-Marques et al. 2004; Wohlrab et al. 2007; Kanan et al 2009; Quinn und Macrae 2011) bieten würden. Solche Hinweise ließen sich durchaus in den Ergebnissen festmachen - wie es bereits in den Ergebnissen zur Hypothese 2 (s. Abschnitt 3.4) im Bezug auf das Schema-Konzept dokumentiert worden ist. Zunächst einmal wurde, wie es das Schema-Konzept erwarten ließ, ein verstärktes Betrachten derjenigen Bildergruppen beobachtet, die als „stark verändert" eingeschätzt worden sind. Das entscheidende daran ist die Unabhängigkeit dieser verlängerten Betrachtungszeiten von der Attraktivitätsbewertung. Maßgeblich für die 
Dauer der Betrachtung einer Veränderung im Gesicht ist stattdessen der Stärkegrad der Abweichung vom "Normalen“, also von dem im Schema gespeicherten „hautgesunden Standardbild“. Wenn man diese zeitliche Veränderung des Betrachtungsverhaltens im Sinne des Schema-Konzeptes deutet, so war auch zu erwarten, dass die zeitlichen Abweichungen bei den hautkranken Bildern besonders in der ersten Phase der Betrachtung die Aufmerksamkeit auf sich ziehen. Auch dieser Effekt ließ sich bestätigen, da die Versuchsteilnehmerinnen signifikant länger während der ersten 15 Sekunden der Bildbetrachtung auf die hautveränderten Areale blickten als in den nachfolgenden 15 Sekunden. Dies ist als eine Art „Neuigkeitseffekt" im Sinne des Schema-Konzeptes zu deuten. Das Neue, Schemafremde, muss zu Beginn verarbeitet bzw. eingearbeitet werden und wird folglich verstärkt aufgesucht. Untermauert wird dies dadurch, dass sich keinerlei vergleichbare Effekte für die hautgesunden Portraitbilder aufzeigen ließen.

Der in dieses kognitive Schema-Konzept passende und von Hünecke (2009) beschriebene "Franzky-Effekt" konnte über alle Bilder gesehen statistisch nur als Trend bestätigt werden $(p<0,07)$. Dieser Effekt beschreibt prinzipiell ein SchemaPriming. Wenn man demnach das Hk-Bild an erster Stelle gesehen hat, „sucht“ man bei Präsentation des Hg-Bildes an zweiter Stelle die gespeicherte "hautkrank"Information (vgl. Befunde von Althoff und Cohen 1999). Für diesen Schema-Ansatz ergibt sich allerdings interessanterweise ein anderes Bild, wenn man einen gezielten Blick allein auf die erste Hälfte der Betrachtung wirft. Für diese Zeitspanne der ersten 15 Sekunden konnte nämlich ein solcher Priming-Effekt in dieser Untersuchung als signifikant nachgewiesen werden.

Andersherum erhielten Hk-Bilder, welche an zweiter Stelle präsentiert wurden einen zusätzlichen Aufmerksamkeitsanstieg für die hautveränderten Areale. Dies entspricht im Grunde einem „doppelten Neuigkeitseffekt", wonach die Hautveränderung nicht nur an sich "ungewöhnlich und neu" erscheint, sondern auch gegenüber den schon zuvor gesehenen bekannten Gesichtsmerkmalen etwas "relativ Neues“ ist (vgl. dazu die aktuelle Arbeit von Hannula et al. 2012).

An diesem Punkt der Diskussion des Schema-Konzepts erscheint es sehr passend, auf einen Nebenbefund dieser Arbeit noch einmal kurz einzugehen, der nicht unmittelbar im visuellen Bereich liegt: In Anlehnung an Greenwald et al. (1998) können kognitive Latenzen bei inkongruenten Urteils-Stimuli auftreten. So waren auch in dieser Untersuchung im Durchschnitt deutlich verlängerte Reaktionszeiten 
bei der Beantwortung nach der "Schwere der Hautveränderung“ festzustellen. Insbesondere galt dies für die Gruppen „eher attraktiv - schwere Veränderung“ und „eher unattraktiv - leichte Veränderung" gegenüber den kongruenten Urteils-Gruppen „eher attraktiv - leichte Hautveränderung“ und „eher unattraktiv - schwere Hautveränderung" (vgl. Abb. 11). Dieser Befund lässt ebenfalls auf innerpsychische Faktoren schließen, die einem rein Reiz-Reaktions-gesteuerten Modell widersprechen. Konkrete Aussagen zur Frage, welche einzelnen Faktoren in welcher Form an diesem Sachverhalt beteiligt sind, lassen sich mit den Werten nicht klären. Allerdings wird hierin ein weiterer wichtiger Anhaltspunkt dafür sehen, die unmittelbare Verknüpfung von Reizgegebenheiten und Wertungen zugunsten eines kognitiv vermittelten Prozesses zu überdenken.

Ein weiterer Schwerpunkt der Studie bestand in der Erforschung eines möglichen Zusammenhangs zwischen der Fixationszeit (FZ) und der kognitiven bzw. der emotionalen Bewertung (Hypothesen-Prüfung 3). Dabei wurde Ersterem eine wesentlich höhere Korrelation unterstellt, und weitergehend von einer signifikant geringeren Korrelation zur emotionalen Bewertung ausgegangen.

Die Ergebnisse konnten dies weitgehend bestätigen, wenn auch vielleicht nicht in der vorweg erhofften Deutlichkeit. So ist der Zusammenhang zwischen FZ und Kognition mit $r=0,45$ (ca. $20 \%$ der Varianz) nahezu doppelt so hoch, wie der Zusammenhang der FZ mit der emotionalen Bewertung, der lediglich 0,35 (ca. 12\% der Varianz) betrug. Ein $t$-Test $(p=0,13)$ konnte allerdings keinen statistisch signifikanten Unterschied bestätigen. Man kann anhand dieser Daten daher nicht sagen, dass es gar keine Verbindung zwischen FZ und emotionaler (Be-)Wertung gibt, allerdings ist der Zusammenhang mit kognitiven Prozessen als deutlich stärker anzusehen (vgl. Acunzo und Henderson 2011; Humphrey et al. 2012).

Innerhalb dieser Bewertungen konnte ein weiteres Phänomen beschrieben werden, das in gewisser Weise einem „Präsentations-Positionseffekt“" entspricht. Dieser Effekt konnte speziell bei Beurteilungen für das Attribut „Attraktivität“ demonstriert werden, wo signifikante Unterschiede in Bezug auf die Reihenfolge der Präsentation auftraten. So ergab sich für die Reihenfolge hautkrank $(\mathrm{Hk})$ - hautgesund $(\mathrm{Hg})$ eine Differenz von ca. einem Rating-Punkt, während sich der Effekt bei der umgekehrten Reihenfolge $(\mathrm{Hg}-\mathrm{Hk})$ nahezu aufhob.

Dieser Effekt kann so interpretiert werden, dass offenbar beim ersten "Kontakt" kritischer hingeschaut wird als beim zweiten. Hierfür sind Deutungen im Rahmen des 
Schema-Konzeptes durchaus möglich und schlüssig. Der Erstkontakt entspricht demnach einer Akkommodation, da noch kein Schema dieser Person oder dieses Gesichtes besteht. Beim zweiten Kontakt muss das bestehende Schema dann lediglich assimiliert werden (vgl. Garcia-Marques et al. 2004; Peskin und Newell 2004). Der nachträgliche Einbau von Hautkrankheiten ins Schema bewirkte dabei allerdings nur eine leichte bis gar keine Veränderung der Attraktivitätseinschätzung. Das Fehlen von vorher bekannten Veränderungen der Haut im Gesicht beim Zweitkontakt, wurde jedoch, wie zuvor bereits beschrieben, mit einer durchschnittlichen Aufwertung von ca. einem Rating-Punkt beurteilt.

Nun hätte man angesichts dieser Ergebnisse einen vergleichbaren Effekt für das Attribut „Auffälligkeit“ erwarten können. In der Summe ließen sich auch durchaus leichte Unterschiede in der Auffälligkeitsbewertung der Hk-Bilder in Abhängigkeit von erster und zweiter Präsentationsstelle (0,23 Ratingpunkte) sowie in den Differenzbeurteilungen zu den $\mathrm{Hg}$-Bildern an erster und zweiter Stelle (0,26 Ratingpunkte) feststellen. Hierin kann ein weiterer Anhaltspunkt für ein Schema-bezogenes Verarbeiten von Gesichtern gesehen werden, da beim Erstkontakt beides - Gesicht und Hautveränderung - neu ist, während beim Zweitkontakt nur die Hautveränderung als neu eingearbeitet werden muss. Diese Differenzen erwiesen sich dennoch als nicht signifikant.

Ein anderer bedeutsamer Teil der Arbeit war ein Nachweis der zu Beginn unterstellten Unabhängigkeit der Dimensionen „Emotion“ und „Kognition“ bei der Bewertung. Dazu wurden jeweils zwei Attribute kombiniert, die zusammen immer eine Dimension repräsentieren sollten; Attraktivität und Angenehmheit die Emotion, Auffälligkeit und Ungewohntheit die Kognition. Den Erwartungen entsprechend bestätigten die Ergebnisse der Faktorenanalyse genau diesen Zusammenhang. Die emotionalen Attribute zeigten eine sehr hohe Ladung auf dem Faktor 2 (jeweils 0,9), während die kognitiven enorm auf dem Faktor 1 luden (Auffälligkeit 0,92, Ungewohntheit 0,93). Ähnlich hohe Werte ergaben sich für die Differenzen in den Bewertungen der $\mathrm{Hg}$ - und Hk-Bilder (Faktor 2: Attraktivität, Angenehmheit jeweils 0,87, Faktor 1: Ungewohntheit 0,87, Auffälligkeit 0,88 ). Somit kann davon ausgegangen werden, dass für die Beurteilungen der Attribute Angenehmheit und Attraktivität einerseits sowie Ungewohntheit und Auffälligkeit, andererseits jeweils unterschiedliche Faktoren eine Rolle gespielt haben. 


\subsection{Ausblicke auf zukünftige Forschung}

Einen weiteren Ansatzpunkt zukünftiger Studien könnte die gezielte Untersuchung männlicher Probanden darstellen, um geschlechtsspezifische Unterschiede im Betrachtungsverhalten genauer zu erforschen, wie sie beispielsweise Hewig et al. (2008) beschrieben. Ein weiterer Aspekt wäre die Erforschung möglicher altersspezifischer Einflussfaktoren bei potentiellen Betrachtern.

Ca. $40 \%$ des Probandenkollektivs gaben an, selbst schon einmal hautkrank gewesen zu sein. Eine detailliertere Verifizierung des Schweregrades der eigenen Hautkrankheit (ob intermittierende Pusteln in der Pubertät oder langjährige Akne mit notwendiger Behandlung in dermatologischen Kliniken) und die Ermittlung möglicher Einflüsse auf das Blick- und Bewertungsverhalten, wie es u. a. Stangier et al. (2008) bei Patienten mit einer Körperdysmorphen Störung (Body-Dismorphic-Disorder) beschrieben haben, wären gleichermaßen interessante Ansatzpunkte für weiterführende Forschung (vgl. Risko et al. 2012).

Speziell für die untersuchten Zusammenhänge wäre es von großem Interesse zu ermitteln, ab wann Personen, hautkrank oder hautgesund, Blicke von anderen Personen als „Anstarren“ empfinden.

Als Beispiel für den „Ausbruch“ dieser Technik aus dem Labor bzw. ihren Einzug ins alltägliche Leben soll an dieser Stelle die Einführung von „Müdigkeitssensoren“ bei führenden Automobilherstellern erwähnt werden. Hier wird mit Hilfe von Blickregistrierung versucht, die Sicherheit im Straßenverkehr zu erhöhen (Niemz 2010).

\subsection{Anwendungsgebiete}

Während differentialdiagnostische Untersuchungsansätze (z.B. Variation des Geschlechtes von Betrachter und Betrachtetem) eine zukünftige Arbeitsrichtung sein können, ist zu prüfen, inwieweit die Ergebnisse der Studie einen psychotherapeutischen Beitrag, insbesondere auch für (kognitive) Verhaltenstherapie, leisten können. Dazu bieten sich u.a. die folgenden drei Befunde dieser Arbeit an: 
- „Digitaler“ Ansatz, d.h. Nutzung von vielen Bildern (Aufnahme und Modifikation [Retusche, Montage, usw.]) und einfache Bildverarbeitung; z.B. kann ein Stigma, in mehrere Bilder - sei es in ein Eigenportrait oder das von anderen Personen - eingesetzt und dargeboten werden.

- Protokollierbarkeit der Bildbetrachtung; z.B. wird schon heute versucht, per Instruktionen auf das Betrachtungsverhalten von Patienten Einfluss zu nehmen, ohne die Auswirkungen bislang objektivieren und kontrollieren zu können (Wilhelm et al. 2011; Trentowska et al. 2013).

- Die größere Bedeutung des kognitiven Aspektes im Vergleich zu emotionalwertenden Einstelllungen beim Betrachtungsverhalten; z.B. könnte dieses Faktenwissen bislang etablierte Kausalattributionsketten beim „AngestarrtWerden" (Abwertung, Ausgrenzung, Stigmatisierung usw.) widerlegen (Hünecke und Bosse 1980; Schubert 1989; Rumsey und Harcourt 2004; Allesch 2006; Hünecke 2009).

So kann der RFV hinsichtlich der Analyse und Behandlung von negativen Denk- und Wahrnehmungsstrukturen, gerade auch bei körperdysmorphen Patienten (Lambrou et al. 2011), im Sinne der Psychoedukation einen sinnvollen Beitrag leisten. 


\section{Zusammenfassung}

Hautveränderungen und Hautkrankheiten können bei betroffenen Personen Gefühle von "Entstellung“ auslösen. Die Beobachtung eines veränderten Betrachtungsverhaltens von Interaktionspartnern oder anderen Personen aus dem persönlichen Umfeld ist in vielen Fällen maßgeblich an der Entstehung solcher Gefühle beteiligt.

In dieser Studie wurde mit Hilfe des Restricted Focus Viewer (RFV) Blickverhalten registriert und detailliert untersucht. Durch eine Analyse von Veränderungen des Blickverhaltens wie auch Korrelationen mit den dazugehörigen Bewertungen sollte das automatisch als abwertend empfundene Betrachtungsverhalten kritisch überprüft werden. Als Grundlage hierfür diente in dieser Arbeit allerdings ein Schemabezogenes Konzept, wonach etwaige Unterschiede beim Erfassen von Veränderungen im Gesicht stärker auf kognitiven als auf emotionalen, wertenden Faktoren beruhen. Um den Einfluss möglicher Störvariablen, wie Geschlecht oder Alter, zu verhindern und damit fundiertere Aussagen machen zu können, wurde das Untersuchungs- und Probandenkollektiv u.a. in diesen Kategorien gezielt eingegrenzt.

Die Erwartungen an die Untersuchung wurden insgesamt bestätigt. So zeigte sich zunächst in der Analyse der Ergebnisse sowie den Auswertungen des Fragebogens, dass der RFV für Untersuchungen auf diesem Gebiet ein geeignetes Verfahren darstellt. Darüber hinaus konnten aus den Daten dieser Arbeit einige neue Erkenntnisse im Bereich der Entstellungsforschung hautkranker Personen gewonnen werden. Viele Aspekte vorausgegangener Studien auf diesem Gebiet, wie beispielsweise ein left gaze bias konnten bestätigt werden.

Als wichtiges stützendes Argument für einen Schema-Konzept-Ansatz konnten "Kognition“ und „Emotion“ als zwei voneinander unabhängige Dimensionen bei der Betrachtung und Bewertung der Portraitbilder herausgestellt werden, wobei sich die verlängerten Blickzeiten deutlich stärker kognitiven Faktoren zuordnen ließen.

Dieser Zusammenhang bestätigte sich darüber hinaus bei Korrelationen von Fixationszeiten (FZ) und Attributen. Hier zeigte sich erwartungsgemäß ein deutlich 
stärkerer Zusammenhang von FZ und dem Attribut Auffälligkeit (kognitiv) gegenüber der Korrelation von FZ und Attraktivität (emotional).

Zusammenfassend liefern die Ergebnisse dieser Untersuchung zahlreiche Anhaltspunkte dafür, Veränderungen des Blickverhaltens im Sinne kognitiver Prozesse zu deuten. Zusätzlich können die gewonnenen Erkenntnisse unter anderem bei der (Psycho-)Therapie von Hautkranken wertvoll sein. Die Studie gibt zudem Anreize für zukünftige Forschung und eine noch effektivere Nutzung des RFV. 


\section{Abkürzungsverzeichnis}

$\begin{array}{ll}\text { AAT } & \text { Attention-Analysis-Tool } \\ \text { EMR } & \text { Eye Mark Recorder } \\ \text { ETS } & \text { Eye-Tracking-System } \\ \text { FWT } & \text { Focus-Window-Technique } \\ \text { FZ } & \text { Fixationszeit/-en } \\ \text { Gr. } & \text { Gruppe } \\ \text { Hg } & \text { hautgesund } \\ \text { Hk } & \text { hautkrank } \\ \text { IAT } & \text { Impliziter Assoziationstest } \\ \text { Mdn } & \text { Median } \\ \text { MRT } & \text { Magnetresonanztomographie } \\ \text { Pbn } & \text { Probanden/-innen } \\ \text { RFV } & \text { Restricted Focus View/-er } \\ \text { SAS } & \text { Statistical Analysis System }\end{array}$

Statistik/Tabellen

df $\quad$ Freiheitsgrade (degree of freedom), relevant für t-Test

p-wert statistische Wahrscheinlichkeit (Grundlage für Signifikanzbeurteilung)

s Streuung

tpaar $t$-Test bei paarweise angeordneten Messwerten aus einer Stichprobe

t-wert Wert des t-Tests 


\section{Anhang}

\section{Bildabfolge}

\begin{tabular}{|c|c|c|c|c|c|c|c|c|c|}
\hline & \multicolumn{9}{|c|}{ Bildabfolge } \\
\hline & \multicolumn{5}{|c|}{ schematisch } & \multicolumn{4}{|c|}{ konkret } \\
\hline \multirow[t]{2}{*}{$\begin{array}{l}\text { Bild- } \\
\text { Nr. }\end{array}$} & $\mathrm{Hg}$ & \multicolumn{2}{|c|}{$\begin{array}{l}\mathrm{Hk} \\
(\mathrm{k})\end{array}$} & \multicolumn{2}{|c|}{$\begin{array}{c}\text { attraktiv } \\
\text { (a) }\end{array}$} & \multicolumn{2}{|c|}{$\begin{array}{c}\text { Versionen 1\&2 } \\
\text { (Version 2: Tausch } \\
\text { 1. \& 2. Hälfte) }\end{array}$} & \multicolumn{2}{|c|}{$\begin{array}{c}\text { Versionen 3\&4 } \\
\text { (Version 4: Tausch } \\
\text { 1. \& 2. Hälfte) }\end{array}$} \\
\hline & & $\begin{array}{c}\text { leicht } \\
\text { (I) }\end{array}$ & $\begin{array}{c}\text { schwer } \\
\text { (s) }\end{array}$ & + & - & 1.Hälfte & 2. Hälfte & 1.Hälfte & 2. Hälfte \\
\hline 1 & G1 & & & $\mathrm{X} 1$ & & 02-al & 05-as & 03-al & 06-as \\
\hline 2 & G2 & & & & $\mathrm{X} 1$ & 10-ul & 12-ul & 11-ul & 09-ul \\
\hline 3 & & K1 & & $\mathrm{X} 2$ & & 01-al-k & 02-al-k & 04-al-k & 03-al-k \\
\hline 4 & G3 & & & X3 & & 03-al & 01-al & 02-al & 04-al \\
\hline 5 & & & $\mathrm{~K} 2$ & $\mathrm{X} 4$ & & 05-as-k & 08-as-k & 06-as-k & 07-as-k \\
\hline 6 & & K3 & & & $\mathrm{X} 2$ & 12-ul-k & 10-ul-k & 09-ul-k & 11-ul-k \\
\hline 7 & G4 & & & & X3 & 11-ul & 09-ul & 10-ul & 12-ul \\
\hline 8 & & $\mathrm{~K} 4$ & & $\times 5$ & & 04-al-k & 03-al-k & 01-al-k & 02-al-k \\
\hline 9 & G5 & & & & $\mathrm{X} 4$ & 13-us & 15-us & 14-us & 16-us \\
\hline 10 & G6 & & & $\mathrm{X6}$ & & 07-as & 06-as & 08-as & 05-as \\
\hline 11 & & & K5 & & X5 & 16-us-k & 14-us-k & 15-us-k & 13-us-k \\
\hline 12 & & K6 & & & X6 & 09-ul-k & 11-ul-k & 12-ul-k & 10-ul-k \\
\hline 13 & & & $\mathrm{~K} 7$ & $\mathrm{X7}$ & & 06-as-k & 07-as-k & 05-as-k & 08-as-k \\
\hline 14 & G7 & & & & $\mathrm{X7}$ & 14-us & 16-us & 13-us & 15-us \\
\hline 15 & & & K8 & & $\mathrm{X} 8$ & 15-us-k & 13-us-k & 16-us-k & 14-us-k \\
\hline 16 & G8 & & & $\mathrm{X} 8$ & & 08-as & 04-al & 07-as & $01-a l$ \\
\hline
\end{tabular}




\section{Fragebogen}

\section{Beurteilung des RFV-Verfahrens}

(Einschätzungen auf 6er-Skalen)

Zu Auswertungszwecken wurden alle Fragen dichotomisiert (1-3 und 3-6).

Die Betrachtungszeit damit war ausreichend.

Die Größe des Maus-Fensters fand ich...

Hinsichtlich der Steuerung des Maus-Fensters fand ich das Negativ-Bild...

Eigentlich genügte bereits das Negativ-Bild, um die jeweilige Person richtig einschätzen zu können.

Könnte ich die Personen unmittelbar betrachten - ohne Negativ und Maus-FensterTechnik -, käme ich zu Eindrücken und Beurteilungen, die wären wahrscheinlich...

\section{Selbstbeurteilungen}

Begegnet man Personen mit Veränderungen an der Haut, ist das Hinschauen auf die Hautstellen unvermeidlich.

Mein Aussehen ist mir ...

Mit meinem Aussehen bin ich ...

Gewöhnlich weiß ich, wie ich aussehe.

Ich mag mein Aussehen so, wie es ist.

Bevor ich außer Haus gehe, schaue ich noch einmal in den Spiegel.

Waren Sie schon einmal hautkrank - oder sind Sie es? 


\section{Literaturverzeichnis}

Acunzo DJ, Henderson JM (2011): No emotional "pop-out" effect in natural scene viewing. Emotion 11 (5), 1134-43

Aharon I, Etcoff N, Ariely D, Chabris CF, O'Connor E, Breiter HC (2001): Beautiful faces have variable reward value: fMRI and behavioural evidence. Neuron $\underline{32}$ (3), 537-551

Allesch CG: Einführung in die psychologische Ästhetik. UTB, Wien 2006

Altabe M, Thompson JK (1996): Body Image: A cognitive self-schema construct? Cognit Ther Res $\underline{20}, 171-193$

Althoff RR, Cohen NJ (1999): Eye-Movement-Based Memory Effect: A Reprocessing Effect in Face Perception. J Exp Psychol Learn Mem Cogn $\underline{25}$ (4), 997-1010

Baker CA (1992): Factors associated with rehabilitation in head and neck cancer. Cancer Nurs $\underline{15}, 395-400$

Barton JJS, Radcliffe N, Cherkasova MV, Edelmann J, Intriligator JM (2006): Information processing during face recognition: The effects of fammilarity, inversion, and morphing on scanning fixations. Perception $\underline{35}, 1089-1105$

Bednarik R, Tukiainen M: Effects of display blurring on the behaviour of novices and experts during program debugging. In: $\mathrm{CHI}$ ' 05 extended abstracts on Human factors in computing systems. ACM Press, New York 2005

Blackwell AF, Jansen AR, Marriott K: Restricted Focus Viewer: A Tool for Tracking Visual Attention. In: Anderson M, Cheng P, Haarslev V (Eds.), Theory and Application of Diagrams. Lecture Notes in Artificial Intelligence 1889. SpringerVerlag, Berlin 2000 
Brady N, Campbell M, Flaherty M (2005): Perceptual asymmetries are preserved in memory for highly familiar faces of self and friend. Brain Cogn $\underline{58}, 334-342$

Broschart S (2009): Know-how - Mouse Tracking: Dem Zeiger auf der Spur: Mit Mousetracking die Website-Usability verbessern. C'T 18,176

Brüninghaus W: Einfluss entstellender Hauterkrankungen auf das Betrachtungsverhalten - experimentelle Analyse mittels Blickrichtungsregistrierung. Med. Diss., Göttingen 1980

Buss DM: Evolutionäre Psychologie. 2. aktualisierte Auflage. Pearson Studium, München, Boston 2004

Cash TF (1989): Body-image affect: Gestalt versus summing the parts. Percept Mot Skills $\underline{69}, 17-18$

Cash TF: The Body-Image workbook: An 8-step program for learning to like your looks. New Harbinger Publications, Oakland 1997

Chen MC, Anderson JR, Sohn MH (2001): What can a mouse cursor tell us more? Correlation of eye/mouse movements on web browsing. Proceedings of $\mathrm{CHI}, 281$ 282

Chun MM, Golomb JD, Turk-Browne NB (2011): A taxonomy of external and internal attention. Annu Rev Psychol 62, 73-101

Daszkowski A: Das Körperbild bei Frauen. Eine empirische Studie über subjektive Körperwahrnehmung unter besonderer Berücksichtigung des Körperbaus. In: Schnittstelle Mensch-Umwelt in Vergangenheit, Gegenwart und Zukunft, Hrsg. v. Schultz M, Henke W Greil H. Cuvillier-Verlag, Göttingen 2000, S. 128-131 
Deco G, Schürmann B (2000): A neuro-cognitive visual system for object recognition based on testing of interactive attentional top-down hypothesis. Perception $\underline{29}, 1249$ 1269

Ekman P: Emotions revealed. Understanding faces and feelings. Weidenfeld und Nicolson-Verlag, London 2003

Fassheber P, Bosse K, Hünecke P, Teichmann AT, Zauner J (1976): Zur sozialen Situation des Haukranken als Phänomen interpersoneller Wahrnehmung: Z Psychosom Med Psychoanal 21, 3-61

Fischer H, Sandblom J, Herlitz A, Fransson P, Wright Cl, Backman L (2004): Sexdifferential brain activation during exposure to female and male faces. Neuroreport $\underline{15}$ (2), 235-238

Foulsham T, Walker E, Kingstone A (2011): The where, what and when of gaze allocation in the lab and the natural environment. Vision Res $\underline{51}$ (17), 1920-1931

Fridlund AJ (1991): Evolution and facial action in reflex, social motive and paralanguage. Biol Psychol $\underline{32}$ (1), 3 -100

Garcia-Marques T, Mackie DM, Claypool HM, Garcia-Marques L (2004): Positivity can cue familarity. Pers Soc Psychol Bull $\underline{30}$, 585-593

Gegenfurtner A, Lehtinen E, Säljö R (2011): Expertise Differences in the Comprehension of Visualizations: a Meta-Analysis of Eye-Tracking Research in Professional Domains. Educ Psychol Rev (2011) 23, 523-552

Greenwald AG, McGhee DE, Schwartz JL (1998): Measuring individual differences in implicit cognition: the implicit association test. J Pers Soc Psychol $\underline{6}, 1468-1480$

Grehn F (2008 a): Augenheilkunde. Lehrbuch der Augenheilkunde, 30. Auflage; Springer-Verlag, Heidelberg 2008, S. $30 \mathrm{ff}$. 
Grehn F (2008 b): Augenheilkunde. Lehrbuch der Augenheilkunde, 30. Auflage; Springer-Verlag, Heidelberg 2008, S. $390 \mathrm{ff}$.

Guo K, Meints K, Hall C, Hall S, Mills D (2009): Left gaze bias in humans, rhesus monkeys and domestic dogs. Anim Cogn $\underline{12}$ (3), 409-418

Guo K, Smith C, Powell K, Nicholls K (2012): Consistent left gaze bias in processing different facial cues. Psychol Res $\underline{76}$ (3), 263-269

Hall JA, Coats EJ, LeBeau LS (2005): Nonverbal behaviour and the vertical dimension of social relations: A meta-analysis. Psychol Bull 131 (6), 898-924

Hannula DE, Baym CL, Warren DE, Cohen NJ (2012): The eyes know: eye movements as a veridical index of memory. Psychol Sci $\underline{23}$ (3), 278-87

Hassebrauck M, Küpper B: Warum wir aufeinander fliegen - die Gesetze der Partnerwahl. Rowohlt-Verlag, Hamburg 2002

Haxby JV, Gobbini MI, Furey ML, Ishai A, Schouten JL, Pietrini P (2001): Distributed and overlapping representation of faces and objects in ventral temporal cortex. Science $\underline{293}$ (5539), 2425-2430

Heinsen S, Vogt P: Usability praktisch umsetzen. Hanser-Verlag, München 2003

Henss R: Gesicht und Persönlichkeitseindruck. (Lehr- und Forschungstexte Psychologie, Bd. 7). Hogrefe-Verlag, Göttingen 1998

Hewig J, Trippe RH, Hecht H, Straube T, Miltner WHR (2008): Gender differences for specific body regions when looking at men and women. Nonverbal Behav $\underline{32}, 67-78$

Hoffman EA, Haxby JV (2000): Distinct representations of eye gaze and identity in the distributed human neural system for face perception. Nat Neurosci $\underline{3}$ (1), 80-84 
Ho-Phuoc T, Guyader N, Landragin F, Guérin-Dugué A (2012): When viewing natural scenes, do abnormal colors impact on spatial or temporal parameters of eye movements? J Vis 12 (2), 4

Hoyningen-Süess U: Einleitung. In: Entstellung und Hässlichkeit. Beiträge aus philosophischer, medizinischer, literatur- und kunsthistorischer sowie aus sonderpädagogischer Perspektive. Hrsg. v. Hoyningen-Süess U, Amrein C. Paul Haupt, Bern, Stuttgart, Wien 1995

Hünecke P: Entstellung: Plädoyer für einen wahrnehmungspsychologischen Ansatz. In: Hauterkrankungen, Psychologische Grundlagen und Behandlung. HogrefeVerlag, Göttingen 2009

Hünecke $\mathrm{P}$, Bosse $\mathrm{K}$ : Entstellung, Erleben und Verarbeitung der äußeren Erscheinung. In: Psychophysiologische Aspekte bei Hautkrankheiten, Hrsg. v. Whitlock FA. Perimed-Verlag, Erlangen 1980

Humphrey K, Underwood G, Lambert T (2012): Salience of the lambs: a test of the saliency map hypothesis with pictures of emotive objects. J Vis 12 (1), 1-15

Jansen AR: Restricted Fokus Viewer (RFV) Version 2.1 User's Manual. Monash University, Australia 2001

Jansen AR, Blackwell AF, Marriott K (2003): A tool for tracking visual attention: The restricted focus viewer. Behav Res Methods Instrum Comput 35, 57-69

Johnson MH, Dzuirawiec S, Ellis H, Morton J (1991): Newborns' preferential tracking of face-like stimuli and its subsequent decline. Cognition $\underline{40}$ (1-2), 1-19

Jones MN, Mewhort DJK (2004): Tracking attention with the focus-window technique: The information filter must be calibrated. Behav Res Methods Instrum Comput $\underline{36}$, 270-276 
Kahle, EA: Aufmerksamkeit für Hautmerkmale - Eine Untersuchung mit dem Restricted Focus Viewer. Psychologische Diplomarbeit, Georg-August-Universität Göttingen 2007

Kanan C, Tong MH, Zhang L, Cottrell GW (2009): SUN: Top-down saliency using natural statistics. Vis cogn $\underline{17}(6-7), 979-1003$

Lambrou C, Veale D, Wilson G (2011): The role of aesthetic sensitivity in body dysmorphic disorder. J Abnorm Psychol 120 (2), 443-53

Leonards U, Scott-Samuel NE (2005): Idiosyncratic initiation of saccadic face exploration in humans. Vision Res $\underline{45}, 2677-2684$

Leppänen J, Hietanen J (2003): Affect and face perception: Odors modulate the recognition advantage of happy faces. Emotion $\underline{3}, 315-326$

Luria SM, Strauss MS (1978): Comparison of eye-movements over faces in photographic positives and negatives. Perception $\underline{7}, 349-358$

Mack A, Rock I: Inattentional blindness. MIT Press, Cambridge 1998

Marc Aurel: Des Kaisers Marcus Aurelius Antonius Selbstbetrachtungen. Übersetzt von Wittstock A; Verlag Philipp Reclam jun. 2007, erschienen im Projekt GutenbergDE. Abrufbar unter http://gutenberg.spiegel.de/buch/1479/5

Mehrabian A, Blum JS (1997): Physical appearance, attractiveness, and the mediating role of emotions. Current Psychology $\underline{16}, 20-43$

Morton J, Johnson MH (1991): CONSPEC and CONLERN: A two-process theory of infant face recognition. Psychol Rev $\underline{98}$ (2), 164-181

Murray JE, Rhodes G, Schuchinski M: When is a face not a face? The effects of misorientation on mechanisms of face perception. In: Perception of faces, objects 
and scenes: Analytic and holistic processes (Advances in visual cognition), Peterson MA und Rhodes G (Eds.). Oxford University Press, New York 2003

Neumann M: Attention Analysis Tool - Entwurf und Implementierung eines mausbasierten Werkzeugs zur Aufmerksamkeitserfassung statischer Szenen. Studienarbeit, FU Berlin, FB Mathematik und Informatik, Januar 2007

Niemz V (2008): Vorrichtung und Verfahren zur blick- und aufmerksamkeitsabhängigen Anpassung des Lenkverhaltens. URL: http://www.freepatentsonline.com/DE102008044075.html (20.8.2011)

Nummenmaa L, Hyönä J, Calvo MG (2006): Eye movement assesment of selective attentional capture by emotional pictures. Emotion $\underline{6}$ (2), 257-268

Nuthmann A, Henderson JM (2010): Object-based attentional selection in scene viewing. J Vis $\underline{10}$ (8), 20

Öhman A: Fear and anxiety as emotional phenomena: Clinical phenomenology, evolutionary perspectives and information-processing mechanisms. In: Handbook of emotions, Lewis M und Haviland JM (Eds.). The Guilford Press, New York, London 1993

O'Reilly C, Plamondon R (2011): Can computer mice be used as low-cost devices for the acquisition of planar human movement velocity signals? Behav Res Methods $\underline{43}$ (1), 229-38

Panse F: Pathophysiologie der Entstellung durch Hautkrankheiten. In: Dermatologie und Venerologie - Ergänzungs- und Registerband, Hrsg. v. Gottron H und Schönfeld W. Thieme, Stuttgart 1970, S. 49-76

Peskin M, Newell FN (2004): Familarity breeds attraction: Effects of exposure on the attractiveness of typical and distinctive faces. Perception $\underline{33}, 147-157$ 
Quinn KA, Macrae CN (2011): The face and person perception: insights from social cognition. Br J Psychol 102 (4), 849-67

Ricciardelli P, Ro T, Driver J (2002): A left visual field advantage in perception of gaze direction. Neuropsychologia $\underline{40}$ (7): 769-77

Risko EF, Kingstone A (2011): Eyes wide shut: implied social presence, eye tracking and attention. Atten Percept Psychophys $\underline{73}$, 291-296

Risko EF, Anderson NC, Lanthier S, Kingstone A (2012): Curious eyes: individual differences in personality predict eye movement behavior in scene-viewing. Cognition $122(1), 86-90$

Rumsey N, Harcourt D (2004): Body-Image and disfigurement: Issues and interventions. Body Image 1, , 83-97

Schneider CF: Bibliothek der Philosophie, Band 2. Phaidon-Verlag, Essen 1996

Schnell R, Hill PB, Esser E: Methoden der empirischen Sozialforschung. 6. überarbeitete Auflage. Oldenbourg, München, Wien 1999

Schubert H-J: Psychosoziale Faktoren bei Hauterkrankungen. Vandenhoeck und Ruprecht, Göttingen 1989

Schweinberger SR, Huddy V, Burton AM (2004): N250r: A face-selective brain response to stimulus repetitions. Neuroreport $\underline{15}$ (9), 1501-1505

Slade PD (1994): What is Body-Image? Behav Res Ther 32, 497-502

Stangier U, Adam-Schwebe S, Müller T, Wolter M (2008): Discrimination of facial appearance stimuli in body dysmorphic disorder. J Abnorm Psychol 117 (2), 435-443 
Thompson JK, Gardner RM: Measuring perceptual Body-Image among adolescents and adults. In: Body-Image - A handbook of theory, research, and clinical practice, Cash TF and Pruzinsky T (Eds.). Guilford-Press, New York 2002

Thornhill R, Grammer K (1999): The body and face of woman: One ornament that signals quality? Evolution and Human Behaviour $\underline{20}$ (2), 105-120

Tollkühn S (2002): Computer mit Blicksteuerung: Gedanken wie Schmetterlinge Dtsch Arztebl $\underline{99}$ (45), 15

Trentowska M, Bender C, Tuschen-Caffier B (2013): Mirror exposure in women with bulimic symptoms: how do thoughts and emotions change in body image treatment? Behav Res Ther $\underline{51}$ (1), 1-6

Wilhelm S, Phillips KA, Fama JM, Greenberg JL, Steketee G (2011): Modular cognitive-behavioral therapy for body dysmorphic disorder. Behav Ther 42 (4), 624633

Wohlrab S, Fink B, Pyritz LW, Rahlfs M, Kappeler PM (2007): Visual attention to plain and ornamented human bodies: An eye tracking study. Percept Mot Skills 104, 13371349

Zerfaß A, Zimmermann $\mathrm{H}$ : Usability von Internet-Angeboten. Grundlagen und Fallstudien. Stuttgarter Beiträge zur Medienwirtschaft Nr.10. Stuttgart, 2004 


\section{Danksagung}

Ich danke Herrn Prof. Dr. med. Thomas Fuchs für die Anregung zu diesem spannenden interdisziplinären Thema und für die Übernahme des Referates.

Außerdem bedanke ich mich bei meinem Betreuer Herrn Dr. phil. Dipl.-Psych. Peter Hünecke für die geduldige Unterstützung während der Planung und Durchführung meiner Arbeit. Er war mit seinen konstruktiven Vorschlägen eine sehr große Hilfe und hatte immer ein offenes Ohr für Fragen und Probleme. Herzlichen Dank für die ausgezeichnete Betreuung.

Ein herzlicher Dank geht auch an Herrn Kaubisch, Fotograf an der Universitätshautklinik Göttingen, der bei der Auswahl des Bildmaterials viele Anregungen einbrachte.

Abschließend bedanke ich mich sehr bei den Probandinnen, die an meiner Arbeit teilnahmen sowie allen Personen, die mich während dieser Zeit persönlich und moralisch unterstützten. 